TRANSACTIONS OF THE

AMERICAN MATHEMATICAL SOCIETY

Volume 362, Number 4, April 2010, Pages 2205-2248

S 0002-9947(09)05032-6

Article electronically published on November 18, 2009

\title{
SMALL-TIME COMPACTNESS AND CONVERGENCE BEHAVIOR OF DETERMINISTICALLY AND SELF-NORMALISED LÉVY PROCESSES
}

\author{
ROSS MALLER AND DAVID M. MASON
}

\begin{abstract}
Consider a Lévy process $X_{t}$ with quadratic variation process $V_{t}=$ $\sigma^{2} t+\sum_{0<s<t}\left(\Delta X_{s}\right)^{2}, t>0$, where $\Delta X_{t}=X_{t}-X_{t-}$ denotes the jump process of $X$. We give stability and compactness results, as $t \downarrow 0$, for the convergence both of the deterministically normed (and possibly centered) processes $X_{t}$ and $V_{t}$, as well as theorems concerning the "self-normalised" process $X_{t} / \sqrt{V_{t}}$. Thus, we consider the stochastic compactness and convergence in distribution of the 2-vector $\left(\left(X_{t}-a(t)\right) / b(t), V_{t} / b(t)\right)$, for deterministic functions $a(t)$ and $b(t)>0$, as $t \downarrow 0$, possibly through a subsequence; and the stochastic compactness and convergence in distribution of $X_{t} / \sqrt{V_{t}}$, possibly to a nonzero constant (for stability), as $t \downarrow 0$, again possibly through a subsequence.

As a main application it is shown that $X_{t} / \sqrt{V_{t}} \stackrel{\mathrm{D}}{\longrightarrow} N(0,1)$, a standard normal random variable, as $t \downarrow 0$, if and only if $X_{t} / b(t) \stackrel{\mathrm{D}}{\longrightarrow} N(0,1)$, as $t \downarrow 0$, for some nonstochastic function $b(t)>0$; thus, $X_{t}$ is in the domain of attraction of the normal distribution, as $t \downarrow 0$, with or without centering constants being necessary (these being equivalent).

We cite simple analytic equivalences for the above properties, in terms of the Lévy measure of $X$. Functional versions of the convergences are also given.
\end{abstract}

\section{INTRODUCTION}

Let $(\Omega, \mathcal{F}, P)$ be a probability space carrying a real-valued Lévy process $\left(X_{t}\right)_{t \geq 0}$, with $X_{0}=0$ and canonical triplet $\left(\gamma, \sigma^{2}, \Pi\right)$, where $\gamma \in \mathbb{R}, \sigma^{2} \geq 0$, and $\Pi$ is a nonnegative measure on $\mathbb{R}$ satisfying $\int_{\mathbb{R} \backslash\{0\}}\left(x^{2} \wedge 1\right) \Pi(\mathrm{d} x)<\infty$. Assume that at least one of $\sigma^{2}$ or $\Pi$ is not identically zero, so $X$ is nontrivial and nondeterministic. The Lévy-Khintchine formula states that $E e^{\mathrm{i} \theta X_{t}}=e^{t \Psi(\theta)}, t \geq 0, \theta \in \mathbb{R}$, where

$$
\Psi(\theta)=\mathrm{i} \gamma \theta-\frac{1}{2} \sigma^{2} \theta^{2}+\int_{\mathbb{R} \backslash\{0\}}\left(e^{\mathrm{i} \theta x}-1-\mathrm{i} \theta x 1_{\{|x| \leq 1\}}\right) \Pi(\mathrm{d} x) .
$$

See [3] or [39]. The jump process of $X$ is $\left(\Delta X_{t}\right)_{t \geq 0}$, where $\Delta X_{t}=X_{t}-X_{t-}, t>0$, and $\Delta X_{0}=0$. Consider also the quadratic variation process

$$
V_{t}=\sigma^{2} t+\sum_{0<s \leq t}\left(\Delta X_{s}\right)^{2}, t>0 .
$$

Studies of the local behavior of Lévy processes have a long and distinguished history, going back to Lévy himself in the 1930s. One line of development, concerned

Received by the editors June 10, 2008 and, in revised form, March 3, 2009.

2000 Mathematics Subject Classification. Primary 60F05, 60F17, 60G51.

The first author's research was partially supported by ARC Grant DP0664603.

The second author's research was partially supported by NSF Grant DMS-0503908. 
with local Hölder-like conditions, can be traced through Blumenthal and Getoor (7) and Pruitt [37, and was recently completed by Bertoin, Doney and Maller [4. Another strand of research investigates weak and strong convergence behavior of Lévy processes at 0 , related to domains of attraction, degenerate convergence, passage-time problems, and laws of the iterated logarithm; see [8, 11, [12, 13, 14.

As a natural development of this, in Mason and Maller 34 we established some foundational results for the (joint) convergence of the deterministically normed (and possibly centered) processes $X_{t}$ and $V_{t}$ to infinitely divisible laws, and a number of results concerning the self-normalised process, $X_{t} / \sqrt{V_{t}}$, were proved in the case of a symmetric $X$. (The process $X_{t}$ is said to be symmetric if $X_{t} \stackrel{\mathrm{D}}{=}-X_{t}$ for each $t>0$.) In particular, it was shown in 34 that, for a symmetric $X, X_{t} / \sqrt{V_{t}}$ is asymptotically standard normal as $t \downarrow 0$ if and only if $X_{t}$ is in the domain of attraction of the normal distribution, as $t \downarrow 0$.

One of the motivations for the present paper was to remove the assumption of symmetry in this result. This may seem a modest aim, but in fact it requires the development of a completely new set of techniques, beyond those in [34. This entailed producing a collection of new theorems concerning compactness, stability, and attraction to normality both of the constant-normed process, and of the selfnormalised process. These have added substantially to our understanding of the small-time behavior of Lévy processes, the final result being a general central limit theorem for the self-normalised process, at 0 .

Our theoretical journey may be compared with the development of the large-time theory of self-normalised random walks. These are the random sequences

$$
T_{n}:=\frac{\xi_{1}+\cdots+\xi_{n}}{\sqrt{\xi_{1}^{2}+\cdots+\xi_{n}^{2}}}
$$

where $\xi, \xi_{1}, \xi_{2}, \ldots$, are i.i.d. real-valued random variables (rvs). The self-normalised process is a natural object to study, being of importance in Statistics and in a variety of areas of applications. Over the last 35 years a considerable number of mathematicians have investigated its asymptotic properties. These include the derivation of central limit theorems, moderate and large deviation theorems, laws of the iterated logarithm, Berry-Esseen theorems, and Edgeworth expansions, etc. For a presentation of the current state of research in these topics consult the monograph by de la Peña, Lai and Shao [10. We shall see that in many ways the small-time theory of Lévy processes parallels that of the self-normalised random walk. (There are also, of course, some crucial differences.)

An outstanding accomplishment of the large-sample analyses of self-normalised random walks was to show that $T_{n}$ is asymptotically standard normal if and only if $\xi$ is in the domain of attraction of the normal distribution, and $E \xi=0$. This proceeded in stages, whose history is enlightening. The problem was first stated as a conjecture in Logan, Mallows, Rice and Shepp [30. In [33] it was pointed out that the "if" part of the conjecture followed from "Raikov's theorem"; cf. Raikov 38, Gut [26]. The conjecture was verified for a symmetric random walk by Roy Erickson. For details see Griffin and Mason [25]. The conjecture without the assumption of symmetry was finally established by Giné, Götze and Mason, [18. Later Mason [35. came up with an independent proof based on results for tightness 
and stochastic compactness of self-normalised sums, which were established in Giné and Mason [19], and Griffin [21].

Guided by the steps developed in [35] and the results in [19] and [21, we can proceed directly to the desired result in the analogous small-time Lévy case. Of course, we must first modify the large-time building blocks constructed in the papers just cited. As could well be expected, major changes are required at most stages. Thus, again, many new results are generated, besides the main objective, which are of importance in themselves, and open up the possibility of some interesting new research.

Our principal theorems are stated in Section 3, along with some related results. Required in their proofs are some subsidiary results on the joint convergence of the processes $X_{t}$ and $V_{t}$, and, especially, on compactness and attraction to normality, as $t \downarrow 0$, of $X_{t}$, possibly after deterministic centering and norming, which are of interest in themselves. These are set out in the next section. All proofs are in Sections 4 and 5 .

The Logan et al. 30] conjecture was wider than we have described; more completely, it asked for necessary and sufficient conditions under which a self-normalised random walk converges in distribution to a nondegenerate random variable. This was finally resolved by Chistyakov and Götze [9. In doing this they showed, among other results, that $T_{n}$ is asymptotically nondegenerate normal (thus, dropping the standard normal requirement) if and only if $\xi$ is in the domain of attraction of the normal distribution. A simple proof of the Logan et al. 30 conjecture in the symmetry case was later discovered by Mason and Zinn in [36]. In [34 we obtained the analogous small time result for self-normalised symmetric Lévy processes. But to address the complete Logan et al. 30] conjecture in a general small time Lévy process setup is well beyond the scope of this paper. We mention some other interesting open questions at the end of Section 3 .

\section{Constant norming compactness at 0 of $X_{t}$ And $V_{t}$}

By the relative compactness of a real-valued stochastic process $\left(S_{t}\right)_{t \geq 0}$, as $t \downarrow 0$, we will mean that it satisfies

$$
\lim _{x \rightarrow \infty} \limsup _{t \downarrow 0} P\left(\left|S_{t}\right|>x\right)=0,
$$

or, equivalently, every sequence $t_{k} \downarrow 0$ contains a subsequence $t_{k^{\prime}} \downarrow 0$ with $S_{t_{k^{\prime}}}$ converging in distribution to an a.s. finite rv. If in addition each such subsequential limit is not degenerate at a constant, we say that $S_{t}$ is stochastically compact, as $t \downarrow 0$.

By the Feller class at 0 we will mean the class of Lévy processes which are stochastically compact at 0 after norming and centering, that is, those for which there are nonstochastic functions $a(t), b(t)>0$ (where, throughout, $b(t)$ will be assumed positive, but not, a priori, monotone), such that every sequence $t_{k} \downarrow 0$ contains a subsequence $t_{k^{\prime}} \downarrow 0$ with

$$
\frac{X_{t_{k^{\prime}}}-a\left(t_{k^{\prime}}\right)}{b\left(t_{k^{\prime}}\right)} \stackrel{\mathrm{D}}{\longrightarrow} Y^{\prime}, \text { as } k^{\prime} \rightarrow \infty
$$

where $Y^{\prime}$ is a finite nondegenerate rv, a.s. (The prime on $Y^{\prime}$ denotes that in general it will depend on the choice of the subsequence $t_{k^{\prime}}$.) We describe this kind of convergence as " $X_{t} \in F C$ at 0 ". 
It was shown in 34 that when the relation (2.1) holds (with $Y^{\prime}$ not degenerate at a constant), then it must be the case that $Y^{\prime}$ is an inf. div. rv, and $b\left(t_{k^{\prime}}\right) \rightarrow 0$ as $t_{k^{\prime}} \downarrow 0$. (We abbreviate "infinitely divisible" to "inf. div." throughout.)

Closely related is the centered Feller class at 0 . This is the class of Lévy processes which are stochastically compact at 0 , after norming, but with no centering function needed, that is, those for which there is a nonstochastic function $b(t)>0$ such that every sequence $t_{k} \downarrow 0$ contains a subsequence $t_{k^{\prime}} \downarrow 0$ with

$$
\frac{X_{t_{k^{\prime}}}}{b\left(t_{k^{\prime}}\right)} \stackrel{\mathrm{D}}{\longrightarrow} Y^{\prime}, \text { as } k^{\prime} \rightarrow \infty
$$

where $Y^{\prime}$ is a finite, nondegenerate, necessarily inf. div., rv, a.s. We describe this as " $X_{t} \in F C_{0}$ at 0 ".

Our first theorem gives criteria for the Feller class $F C$ at 0 in terms of the canonical measure of $X$, including also results on the convergence of the quadratic variation process $V$, joint with $X$. Recall that we denote the canonical triplet of $X_{t}$ by $\left(\gamma, \sigma^{2}, \Pi\right)$. The tails $\bar{\Pi}(x)$ and $\bar{\Pi}^{ \pm}(x)$ of $\Pi$ are defined by $(2.3)$

$\bar{\Pi}^{-}(x)=\Pi\{(-\infty,-x)\}, \bar{\Pi}^{+}(x)=\Pi\{(x, \infty)\}$, and $\bar{\Pi}(x)=\bar{\Pi}^{+}(x)+\bar{\Pi}^{-}(x), \quad x>0$.

We will also use some truncated mean and variance functions, defined for $x>0$ by

$$
\nu(x)=\gamma-\int_{x<|y| \leq 1} y \Pi(\mathrm{d} y), V(x)=\sigma^{2}+\int_{|y| \leq x} y^{2} \Pi(\mathrm{d} y),
$$

and

$$
U(x)=\sigma^{2}+2 \int_{0}^{x} y \bar{\Pi}(y) \mathrm{d} y .
$$

These functions are finite for all $x>0$ by virtue of the properties of the Lévy measure $\Pi$, which further imply that $\lim _{x \downarrow 0} x^{2} \bar{\Pi}(x)=0$, and, as is easily verified, $\lim _{x \downarrow 0} x \nu(x)=0$.

It is shown in Theorem 2.1 of [34] that the joint characteristic function of $\left(X_{t}, V_{t}\right)$ is given by

$$
\begin{aligned}
E e^{\mathrm{i}\left(\theta_{1} X_{t}+\theta_{2} V_{t}\right)}= & e^{\mathrm{i} \theta_{1} \gamma t-t \theta_{1}^{2} \sigma^{2} / 2} \times e^{\mathrm{i} t \theta_{2}\left(\sigma^{2}+\int_{|x| \leq 1} x^{2} \Pi(\mathrm{d} x)\right)} \\
& \times e^{t \int_{\mathbb{R}}\left(e^{\mathrm{i}\left(\theta_{1} x+\theta_{2} x^{2}\right)}-1-\mathrm{i}\left(\theta_{1} x+\theta_{2} x^{2}\right) \mathbf{1}_{\{|x| \leq 1\}}\right) \Pi(\mathrm{d} x)},
\end{aligned}
$$

where $\theta_{1}, \theta_{2} \in \mathbb{R}$ and $t \geq 0$.

In Section 3 we will need to divide out $\sqrt{V_{t}}$, to get the self-normalised process. Now $P\left(V_{t}=0\right)=0$ for each $t>0$ if and only if $\sigma^{2}>0$ or $\bar{\Pi}(0+)=\infty$ (see Lemma 4.3 in [34). We will exclude throughout the case $\bar{\Pi}(0+)<\infty$, since otherwise $X$ is Brownian motion with drift in a neighbourhood of 0 , and $V_{t}=\sigma^{2} \geq 0$ there, so the answers to the questions we investigate later will be obvious. Thus we assume throughout that $\bar{\Pi}(0+)=\infty$. Under this assumption, $\Pi$ has points of increase arbitrarily close to 0 , so $V(x)>0$ for all small $x>0$; also, $X_{t}$ has jumps arbitrarily close to 0 , and $V_{t}>0$ a.s. for each $t>0$ (whether or not $\sigma^{2}>0$ ). We further have $\bar{\Pi}(x)>0$ for all small $x>0$, so by rescaling if necessary we can take $\bar{\Pi}(x)>0$ for all $x$ in $[0,1)$.

Our first theorem gives the small-time Lévy analogue of the Feller class, including the joint convergence of $X_{t}$ and $V_{t}$. See Bingham et al. [5], p.54, for the dominated variation concepts, and let $\delta_{x}$ denote a point mass at $x \in \mathbb{R}$. 
Theorem 2.1. The following are equivalent:

(i) $X_{t} \in F C$ at 0 .

(ii) There are nonstochastic functions $a(t), b(t)>0$, such that every sequence $t_{k} \downarrow 0$ contains a subsequence $t_{k^{\prime}} \downarrow 0$ with

$$
\left(\frac{X_{t_{k^{\prime}}}-a\left(t_{k^{\prime}}\right)}{b\left(t_{k^{\prime}}\right)}, \frac{V_{t_{k^{\prime}}}}{b^{2}\left(t_{k^{\prime}}\right)}\right) \stackrel{\mathrm{D}}{\longrightarrow}\left(\mathcal{I}^{\prime}, \mathcal{J}^{\prime}\right), \text { as } k^{\prime} \rightarrow \infty
$$

where $\left(\mathcal{I}^{\prime}, \mathcal{J}^{\prime}\right)$ is a bivariate infinitely divisible random 2-vector having canonical triplet $\left(\gamma_{\mathcal{I}^{\prime}, \mathcal{J}^{\prime}}, \Sigma_{\mathcal{I}^{\prime}, \mathcal{J}^{\prime}}, \Lambda_{\mathcal{I}^{\prime}, \mathcal{J}^{\prime}}\right)$, with $\gamma_{\mathcal{I}^{\prime}, \mathcal{J}^{\prime}}=\left(0,\left(\tau^{\prime}\right)^{2}\right)$, for a constant $\left(\tau^{\prime}\right)^{2} \geq 0$, $\Sigma_{\mathcal{I}^{\prime}, \mathcal{J}^{\prime}}$ a $2 \times 2$ matrix with upper diagonal element $\left(\tau^{\prime}\right)^{2}$ and all other elements 0 , and $\Lambda_{\mathcal{I}^{\prime}, \mathcal{J}^{\prime}}$ a bivariate Lévy measure concentrated on the parabola $\{(x, v) \in \mathbb{R} \times[0, \infty)$ : $\left.v=x^{2}\right\}$. Let $\Lambda^{\prime}$ be the one-dimensional projection on the $x$-axis of the bivariate Lévy measure $\Lambda_{\mathcal{I}^{\prime}, \mathcal{J}^{\prime}}$. Then there are constants $c \geq 1$ and $\alpha \in(0,2]$, not depending on the choice of subsequence, such that the tail $\bar{\Lambda}^{\prime}(x)$ of the Lévy measure $\Lambda^{\prime}(x)$ satisfies $\bar{\Lambda}^{\prime}(x) \leq c x^{-\alpha}$ for all $x>1$; also, $\tau^{\prime}>0$ or $\bar{\Lambda}^{\prime}(0+)=\infty$. Further, $\mathcal{I}^{\prime}$ is not degenerate at any constant, $\mathcal{J}^{\prime}$ has canonical triplet $\left(0,0, \Lambda_{\mathcal{J}^{\prime}}\right)$, where $\Lambda_{\mathcal{J}^{\prime}}$ satisfies $\bar{\Lambda}_{\mathcal{J}^{\prime}}(x)=\bar{\Lambda}^{\prime}(\sqrt{x}), x>0$, and $\left.P\left(\left(\tau^{\prime}\right)^{2}\right)+\mathcal{J}^{\prime} \leq 0\right)=0$.

(iii) There is a nonstochastic function $b(t)>0$ such that every sequence $t_{k} \downarrow 0$ contains a subsequence $t_{k^{\prime}} \downarrow 0$ with

$$
\frac{V_{t_{k^{\prime}}}}{b^{2}\left(t_{k^{\prime}}\right)} \stackrel{\mathrm{D}}{\longrightarrow} \mathcal{K}^{\prime}, \text { as } k^{\prime} \rightarrow \infty
$$

where $\mathcal{K}^{\prime}$ is a finite rv a.s., with $P\left(\mathcal{K}^{\prime} \leq 0\right)=0$.

(iv)

$$
\limsup _{x \downarrow 0} \frac{x^{2} \bar{\Pi}(x)}{V(x)}<\infty .
$$

When the conditions of the theorem hold, the function $b(t)$ in (2.6) can be chosen to be continuous and strictly increasing, with $b(0)=0$. The function $a(t)$ satisfies $a(t)=t \nu(b(t))+O(b(t))$ as $t \downarrow 0$.

If $\sigma^{2}>0$, then $b(t) / \sqrt{t} \rightarrow \sigma$ and $\left(X_{t} / \sqrt{t}, V_{t} / t\right) \stackrel{\mathrm{D}}{\longrightarrow}\left(N\left(0, \sigma^{2}\right), \delta_{\sigma^{2}}\right)$, as $t \downarrow 0$. If $\sigma^{2}=0$, then $b(t) / \sqrt{t} \rightarrow 0$ as $t \downarrow 0$. In either case, $b(t)$ varies dominatedly at 0 in the sense that $\limsup _{t \downarrow 0} b(\lambda t) / b(t) \leq \lambda^{1 / \alpha}$ for some $\alpha \in(0,2]$, for all $\lambda>1$, and both $V(x)$ and $U(x)$ vary dominatedly at 0 , with $\lim _{\sup _{x \downarrow 0}} V(\lambda x) / V(x) \leq C \lambda^{2-\alpha}$ for some $C>0$, for all $\lambda>1$, and $\lim \sup _{x \downarrow 0} U(\lambda x) / U(x) \leq \lambda^{2-\alpha}$.

We can strengthen the convergence in Theorem 2.1 to a functional version. Let $D_{0}[0,1]$ be the càdlàg functions on $[0,1]$ which are 0 at 0.

Theorem 2.2. Suppose (2.8) holds. Then there is a nonstochastic function $b(t)>0$ such that every sequence $t_{k} \downarrow 0$ contains a subsequence $t_{k^{\prime}} \downarrow 0$ for which the bivariate process

$$
\left(\frac{X_{y t}-y t \nu(b(t))}{b(t)}, \frac{V_{y t}}{b^{2}(t)}\right)_{0 \leq y \leq 1},
$$

indexed by $t>0$, converges weakly as $t \downarrow 0$ through the subsequence $t_{k^{\prime}}$, in the Skorokhod topology on $D_{0}[0,1] \times D_{0}[0,1]$, to a bivariate Lévy process $\left(\mathcal{I}^{\prime}(y), \mathcal{J}^{\prime}(y)\right)_{y \geq 0}$, having canonical triplet $\left(\gamma_{\mathcal{I}^{\prime}, \mathcal{J}^{\prime}}, \Sigma_{\mathcal{I}^{\prime}, \mathcal{J}^{\prime}}, \Lambda_{\mathcal{I}^{\prime}, \mathcal{J}^{\prime}}\right)$, as described in Theorem 2.1. The 
process $\left(\mathcal{I}^{\prime}(y), \mathcal{J}^{\prime}(y)\right)_{y \geq 0}$ is such that

$\Delta \mathcal{J}^{\prime}(y)=\mathcal{J}^{\prime}(y)-\mathcal{J}^{\prime}(y-)=\left(\Delta \mathcal{I}^{\prime}(y)\right)^{2}=\left(\mathcal{I}^{\prime}(y)-\mathcal{I}^{\prime}(y-)\right)^{2}$, for each $y \geq 0$.

The next theorem characterises the class $F C_{0}$.

Theorem 2.3. The following are equivalent:

(i) $X_{t} \in F C_{0}$ at 0 .

(ii) There is a nonstochastic function $b(t)>0$ such that every sequence $t_{k} \downarrow 0$ contains a subsequence $t_{k^{\prime}} \downarrow 0$ for which the bivariate process

$$
\left(\frac{X_{y t}}{b(t)}, \frac{V_{y t}}{b^{2}(t)}\right)_{0 \leq y \leq 1}
$$

indexed by $t>0$, converges weakly as $t \downarrow 0$ through the subsequence $t_{k^{\prime}}$, in the Skorokhod topology on $D_{0}[0,1] \times D_{0}[0,1]$, to a bivariate Lévy process $\left(\mathcal{I}^{\prime}, \mathcal{J}^{\prime}\right)$ as described in Theorem 2.2, except that the shift 2-vector $\gamma_{\mathcal{I}^{\prime}, \mathcal{J}^{\prime}}$ now has the form $\left(\beta^{\prime},\left(\tau^{\prime}\right)^{2}\right)$, for a constant $\beta^{\prime} \in \mathbb{R}$.

$$
\limsup _{x \downarrow 0} \frac{x^{2} \bar{\Pi}(x)+x|\nu(x)|}{V(x)}<\infty .
$$

Remarks. (i) Provided we take $x \uparrow \infty$ rather than $x \downarrow 0$ in them, and make appropriate correspondences between the functions $\bar{\Pi}(x), \nu(x)$ and $V(x)$, and functionals of the cdf of the increments of a random walk, conditions (2.8) and (2.11) are precisely the necessary and sufficient conditions for the random walk to be in $F C$ or $F C_{0}$, where now the compactness is interpreted as being for large values of the time parameter; see for example Feller [15, Giné and Mason [19], Griffin and Maller [22, 23. However, the extra convergences concerning $V_{t}$ are usually not mentioned in this context.

(ii) Conditions (i)-(iii) in Theorem 2.3 remain equivalent if the convergence in Part (ii) is only required to hold for $y=1$, just as in Theorem 2.1.

The next theorem characterises attraction to normality of $X_{t}$ at 0 . Write $X_{t} \in$ $D(N)$ at 0 if there are nonstochastic functions $a(t), b(t)>0$, such that

$$
\frac{X_{t}-a(t)}{b(t)} \stackrel{\mathrm{D}}{\longrightarrow} \tau Z \text { as } t \downarrow 0,
$$

where $\tau>0$ is a constant and $Z$ is a standard normal rv, and say that $X_{t} \in D_{0}(N)$ at 0 if (2.12) holds with $a(t) \equiv 0$.

Theorem 2.4. The following are equivalent:

(i) $X_{t} \in D(N)$ at 0 .

(ii)

$$
\lim _{x \downarrow 0} \frac{x^{2} \bar{\Pi}(x)}{V(x)}=0 .
$$

(iii) There are nonstochastic functions $a(t), b(t)>0$, such that every sequence $t_{k} \downarrow 0$ contains a subsequence $t_{k^{\prime}} \downarrow 0$ with

$$
\frac{X_{t_{k^{\prime}}}-a\left(t_{k^{\prime}}\right)}{b\left(t_{k^{\prime}}\right)} \stackrel{\mathrm{D}}{\longrightarrow} \tau^{\prime} Z \text {, as } k^{\prime} \rightarrow \infty,
$$

where $\tau^{\prime}>0$ is a constant and $Z$ is a standard normal $r v$. 
(iv) There is a nonstochastic function $b(t)>0$ such that every sequence $t_{k} \downarrow 0$ contains a subsequence $t_{k^{\prime}} \downarrow 0$ with

$$
\frac{X_{t_{k^{\prime}}}}{b\left(t_{k^{\prime}}\right)} \stackrel{\mathrm{D}}{\longrightarrow} \tau^{\prime} Z, \text { as } k^{\prime} \rightarrow \infty
$$

where $\tau^{\prime}>0$ is a constant and $Z$ is a standard normal $r v$.

(v) $X_{t} \in D_{0}(N)$ at 0 .

(vi)

$$
\lim _{x \downarrow 0} \frac{x^{2} \bar{\Pi}(x)+x|\nu(x)|}{V(x)}=0 .
$$

(vii) There is a nonstochastic function $b(t)>0$ such that every sequence $t_{k} \downarrow 0$ contains a subsequence $t_{k^{\prime}} \downarrow 0$ with

$$
\frac{V_{t_{k^{\prime}}}}{b^{2}\left(t_{k^{\prime}}\right)} \stackrel{\mathrm{P}}{\longrightarrow}\left(\tau^{\prime}\right)^{2}, \text { as } k^{\prime} \rightarrow \infty
$$

where $\left(\tau^{\prime}\right)^{2} \in(0, \infty)$ is a constant.

When (i)-(vii) hold, the function $b(t)$ can be chosen to be continuous and strictly increasing, with $b(0)=0$, and thus $b(t) \downarrow 0$ as $t \downarrow 0$. Also, $b(t)$ is regularly varying with index $1 / 2$ as $t \downarrow 0$, and $a(t)=o(b(t))$ as $t \downarrow 0$. The functions $V(x)$ and $U(x)$ are slowly varying as $x \downarrow 0$.

Remarks. (i) There are functional versions of (2.12) and for $D_{0}(N)$, with $a(t) \equiv 0$, and similarly in Theorem 3.6 below, where the limiting process is a nondegenerate Brownian motion. These follow in just the same way as for Theorems 2.1 and 2.2

(ii) The equivalence of $D_{0}(N)$ with (2.16) is in Theorem 2.5 of [11, but the extra subsequential equivalences in (2.14) and (2.15) were not given there, nor was $V_{t}$ considered. The distinguishing feature of $D_{0}(N)$, as compared with $D(N)$, ostensibly, is that no centering function is necessary, but as Theorem 2.4 shows, in fact $D_{0}(N)=D(N)$ (at zero!). This was not noticed in 11]. In [13, the class $D_{0}(N)$ is connected with the stability of the two-sided exit time $T(r):=\inf \{t>$ $\left.0:\left|X_{t}\right|>r\right\}$, for $r>0$.

(iii) It might be enquired why we don't allow centering of the quadratic variation process $\left(V_{t}\right)_{t \geq 0}$, in Theorem 2.1, and elsewhere. This would not be appropriate for the applications to follow in Section 3, as we require the limit of the normalised $V_{t}$ to stay positive. The question may have some interest otherwise, but we do not investigate it further here.

In what follows we denote the class of slowly varying functions by " $S V$ ", with a descriptor "at 0" or "at infinity" as appropriate. Likewise, functions which are regularly varying with index $\alpha$ are in " $R V(\alpha)$ " (at 0 or at infinity, as appropriate).

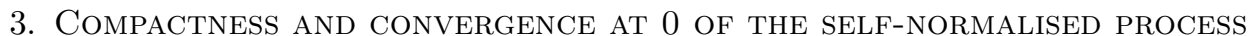

Our main result in this section is Theorem 3.6, which gives a central limit theorem for the self-normalised process $X_{t} / \sqrt{V_{t}}$ at 0 . As an intermediate step in its proof, we need an equivalence for the relative compactness of $X_{t} / \sqrt{V_{t}}$. This is a "small time" analogue of a key result of Griffin 21. We state this first, then give a criterion for the stability of $X_{t} / \sqrt{V_{t}}$ as $t \downarrow 0$, i.e., for when $X_{t} / \sqrt{V_{t}} \stackrel{\mathrm{P}}{\longrightarrow} c$ as $t \downarrow 0$ for a finite nonzero constant $c$. Recall our blanket assumption that $\bar{\Pi}(0+)=\infty$, as a result of 
which $V_{t}>0$ a.s. for all $t>0$ and $V(x)>0$ for all $x>0$, and similarly for $U(x)$ (see (2.4) ).

Theorem 3.1. We have that

$$
\frac{X_{t}}{\sqrt{V_{t}}} \text { is relatively compact as } t \downarrow 0 \text { if and only if } \limsup _{x \downarrow 0} \frac{x|\nu(x)|}{U(x)}<\infty \text {. }
$$

Remark. $X_{t} / \sqrt{V_{t}}$ is not always relatively compact. For example, from Proposition 4.1 in Section 4 , we can deduce that

$$
\left(\frac{X_{t}}{t}, \frac{V_{t}}{t^{2}}\right) \stackrel{\mathrm{P}}{\longrightarrow}(\delta, 0), \text { as } t \downarrow 0
$$

if $X_{t}$ is a subordinator with drift $\delta$. Thus, if $\delta \neq 0, X_{t} / \sqrt{V_{t}}$ is not relatively compact at 0 . As another kind of example, take $\sigma^{2}=0$ and $\Pi$ so that $\bar{\Pi}(x)=$ $\mathbf{1}_{\left\{0<x<e^{-1}\right\}} /(x|\log x|)$. Then $\nu(x) \sim \log (|\log x|)$, and $U(x) \sim 2 x /|\log x|$, as $x \downarrow 0$, so $\lim _{x \downarrow 0} x|\nu(x)| / U(x)=\infty$, and again $X_{t} / \sqrt{V_{t}}$ is not relatively compact at 0 .

However, $X_{t} / \sqrt{V_{t}}$ can always be rendered relatively compact by deterministic centering; see Corollary 5.2 in Section 5 .

Since $\nu(x) \equiv 0$ when $X_{t}$ is symmetric, we see that, then, $X_{t} / \sqrt{V_{t}}$ is always relatively compact as $t \downarrow 0$. In fact, if $X_{t}$ is symmetric, $X_{t} / \sqrt{V_{t}}$ is always stochastically compact, as $t \downarrow 0$ :

Theorem 3.2. Suppose $X_{t}$ is symmetric. Then $X_{t} / \sqrt{V_{t}}$ is stochastically compact, as $t \downarrow 0$. In particular, there is no subsequence $t_{k} \downarrow 0$ such that $X_{t_{k}} / \sqrt{V_{t_{k}}} \stackrel{\mathrm{P}}{\longrightarrow} c$, for a finite or infinite constant $c$.

Remark. After the proof of Theorem 3.2, we remark that $X_{t_{k}} / \sqrt{V_{t_{k}}} \stackrel{\mathrm{P}}{\longrightarrow} 0$ is not possible for any $t_{k} \downarrow 0$, whether or not $X$ is symmetric. We also have

Theorem 3.3. Let $X_{t} \in F C_{0}$ at 0 . Then each sequence $t_{k} \downarrow 0$ contains a subsequence $t_{k^{\prime}} \downarrow 0$ for which $X_{t_{k^{\prime}}} / \sqrt{V\left(t_{k^{\prime}}\right)}$ converges in distribution to an a.s. finite $r v$, and the distribution of any such subsequential limit $r v$ cannot be concentrated on a finite number of points; in particular, cannot be degenerate. Thus, $X_{t} / \sqrt{V_{t}}$ is stochastically compact at 0 .

As part of studying the compactness properties of $X_{t} / \sqrt{V_{t}}$, we need to investigate its possible degenerate limits. The next theorem looks at the stability in probability of $X_{t} / \sqrt{V_{t}}$. It shows that this kind of behavior is restricted to a subset of bounded variation processes.

Theorem 3.4. The following are equivalent:

(a) $\sigma^{2}=0, \bar{\Pi}(x) \in S V$ at $0, X$ is of bounded variation with no drift, and $\bar{\Pi}^{-}(x)=o\left(\bar{\Pi}^{+}(x)\right)$, as $x \downarrow 0$;

(b) $X_{t} / \sqrt{V_{t}} \stackrel{\mathrm{P}}{\longrightarrow}+1$, as $t \downarrow 0$;

(c) $X_{t} / \sqrt{V_{t}} \stackrel{\mathrm{P}}{\longrightarrow}$ c, as $t \downarrow 0$, for a constant $c \in(0, \infty)$.

Remarks (Applications). The case when $\bar{\Pi}(x) \in S V$ at 0 is by no means an anomalous one, in practice. The variance gamma model (31), widely used in financial modelling, has Lévy density

$$
\Pi(\mathrm{d} x)=\frac{1}{|x| \nu} e^{\alpha x / \beta^{2}} e^{\frac{-|x|}{\beta} \sqrt{\frac{2}{\nu}+\frac{\alpha^{2}}{\beta^{2}}}} \mathrm{~d} x
$$


for $x \in \mathbb{R} \backslash\{0\}$, and certain parameters $\nu>0, \alpha>0$, and $\beta>0$. Thus $\bar{\Pi}(x)$ has a logarithmic singularity at 0 and in fact is $S V$ at 0 . Other Lévy processes are also used in financial modelling, see [1, and a discretised version of self-normalisation of the process is a useful statistical tool; see for example the references in [34. 40] gives a good recent overview of estimation issues to do with the " $p$-variation" of a time-changed Lévy process. We expect that the issue of small-time behavior of the self-normalised process will be of interest in these applications (cf. also [6]).

Corollary 3.5 (Corollary to Theorem $\underline{3.4}$ ). $\left(X_{t}-a(t)\right) / \sqrt{V_{t}} \stackrel{\mathrm{P}}{\longrightarrow}+1$, as $t \downarrow 0$, for a deterministic function $a(t)$, if and only if $\sigma^{2}=0, \bar{\Pi}(x) \in S V$ at $0, \bar{\Pi}^{-}(x)=$ $o\left(\bar{\Pi}^{+}(x)\right)$, as $x \downarrow 0$, and $a(t)=t \delta+o_{P}\left(\sqrt{V_{t}}\right)$, as $t \downarrow 0$, where $\delta$ is the drift of $X$.

By sign reversal we get a version of Theorem 3.4 with $X_{t} / \sqrt{V_{t}} \stackrel{\mathrm{P}}{\longrightarrow}-1$, as $t \downarrow 0$, and $\bar{\Pi}^{+}(x)=o\left(\bar{\Pi}^{-}(x)\right)$, as $x \downarrow 0$, in Part (a), and with $c \in(-\infty, 0)$ in Part (c), and similarly in the corollary. Next comes our main result in this section.

Theorem 3.6. We have $X_{t} / \sqrt{V_{t}} \stackrel{\mathrm{D}}{\longrightarrow} N(0,1)$ as $t \downarrow 0$ if and only if $X_{t} \in D_{0}(N)$ at 0 .

Remarks (Concluding Remarks). Our investigation opens many interesting new lines of enquiry. For example: from Lemma 5.3 in Section 5 we see that $\sigma^{2}=0$ and $\bar{\Pi} \in S V$ at 0 imply $\left|X_{t}\right| / \sqrt{V_{t}} \stackrel{\mathrm{P}}{\longrightarrow} 1$ as $t \downarrow 0$. Is the converse true?

It would be useful to allow centering in Theorem 3.6. does $\left(X_{t}-a(t)\right) / \sqrt{V_{t}} \stackrel{\mathrm{D}}{\longrightarrow}$ $N(0,1)$, as $t \downarrow 0$, for a given deterministic function $a(t)$, imply $X_{t} \in D_{0}(N)$ at 0 ?

Can we give necessary and sufficient conditions for $X_{t} / \sqrt{V_{t}}$ to be stochastically compact at 0 ? For this, a subsequential version of Theorem 3.4 would be useful.

As we mentioned in Section [1 currently unattempted is to characterise the possible limit laws and domains of attraction of $X_{t} / \sqrt{V_{t}}$ and $\left(X_{t}-a(t)\right) / \sqrt{V_{t}}$ as $t \downarrow 0$. The reader will think of many more possibilities.

\section{Proofs for Section 2}

Recall the formal setup in Section 1, In the sequel we will often use the following decomposition, or a variant of it, which can be obtained from (1.1): for any $b>0$ we can write

$$
X_{t}=t \nu(b)+\sigma Z_{t}+X_{t}^{(S, b)}+X_{t}^{(B, b)}, t \geq 0,
$$

where $Z_{t}$ is a standard Brownian motion (SBM), $X_{t}^{(S, b)}$ is the compensated sum of "small" jumps, i.e.

$$
X_{t}^{(S, b)}=\text { a.s. } \lim _{\varepsilon \downarrow 0}\left(\sum_{0<s \leq t} \Delta X_{s} 1_{\left\{\varepsilon<\left|\Delta X_{s}\right| \leq b\right\}}-t \int_{\varepsilon<|x| \leq b} x \Pi(\mathrm{d} x)\right), t \geq 0,
$$

and $X_{t}^{(B, b)}$ denotes the "big" jumps, i.e.,

$$
X_{t}^{(B, b)}=\sum_{0<s \leq t} \Delta X_{s} 1_{\left\{\left|\Delta X_{s}\right|>b\right\}}, t \geq 0 .
$$

Further, the processes $\left(Z_{t}\right)_{t \geq 0},\left(X_{t}^{(S, b)}\right)_{t \geq 0}$ and $\left(X_{t}^{(B, b)}\right)_{t \geq 0}$ are all independent. 
The following preliminary proposition, and its corollary, concerning functional convergence of $X_{t}$ and $V_{t}$, after deterministic norming (and centering, for $X_{t}$ ), generalise Theorem 2.2 and Corollary 2.1 of [34].

Proposition 4.1. Suppose there are nonstochastic sequences $t_{k} \downarrow 0, b_{k}>0, b_{k} \rightarrow 0$ as $k \rightarrow \infty$, such that

$$
\lim _{k \rightarrow \infty} t_{k} \bar{\Pi}^{ \pm}\left(x b_{k}\right)=\bar{\Lambda}^{ \pm}(x), x>0
$$

at continuity points of $\bar{\Lambda}^{ \pm}$, where $\Lambda$ is a Lévy measure with tails defined as in (2.3); and, for some $\tau^{2} \in[0, \infty)$,

$$
\lim _{\varepsilon \downarrow 0} \liminf _{k \rightarrow \infty} \frac{t_{k} V\left(\varepsilon b_{k}\right)}{b_{k}^{2}}=\lim _{\varepsilon \downarrow 0} \limsup _{k \rightarrow \infty} \frac{t_{k} V\left(\varepsilon b_{k}\right)}{b_{k}^{2}}=\tau^{2} .
$$

Then as $k \rightarrow \infty$, the processes

$$
\left(\mathcal{I}_{t_{k}}(y), \mathcal{J}_{t_{k}}(y)\right)_{0 \leq y \leq 1}:=\left(\frac{X_{y t_{k}}-y t_{k} \nu\left(b_{k}\right)}{b_{k}}, \frac{V_{y t_{k}}}{b_{k}^{2}}\right)_{0 \leq y \leq 1}
$$

converge weakly in the Skorokhod topology on $D_{0}[0,1] \times D_{0}[0,1]$ to a bivariate Lévy process $(\mathcal{I}(y), \mathcal{J}(y))_{y \geq 0}$, where $(\mathcal{I}(y))_{y \geq 0}$ is Lévy with canonical triplet $\left(0, \tau^{2}, \Lambda\right)$, and $(\mathcal{J}(y))_{y \geq 0}$ is Lévy with canonical triplet $\left(\tau^{2}, 0, \Lambda_{\mathcal{J}}\right)$, with $\bar{\Lambda}_{\mathcal{J}}(x)=\bar{\Lambda}(\sqrt{x})$ for $x>0$, and is such that

$$
\Delta \mathcal{J}(y)=\mathcal{J}(y)-\mathcal{J}(y-)=(\Delta \mathcal{I}(y))^{2}=(\mathcal{I}(y)-\mathcal{I}(y-))^{2}, \text { for each } y \geq 0 .
$$

Conversely, suppose there exist nonstochastic sequences $t_{k} \downarrow 0, a_{k} \in \mathbb{R}$ and $b_{k}>$ $0, b_{k} \rightarrow 0$ as $k \rightarrow \infty$, such that $\left(X_{t_{k}}-a_{k}\right) / b_{k} \stackrel{\mathrm{D}}{\longrightarrow} Y$, where $Y$ is a finite rv, a.s. Then $Y$ is an inf. div. rv with canonical triplet $\left(\beta, \tau^{2}, \Lambda\right)$, for some constants $\beta \in \mathbb{R}$ and $\tau^{2} \geq 0$, and some Lévy measure $\Lambda$; further, (4.2) and (4.3) hold, and $a_{k}$ satisfies

$$
a_{k}=t_{k} \nu\left(b_{k}\right)-b_{k} \beta+o\left(b_{k}\right) \text {, as } k \rightarrow \infty .
$$

Corollary 4.2. Suppose there are nonstochastic sequences $t_{k} \downarrow 0, b_{k}>0, b_{k} \rightarrow 0$ as $k \rightarrow \infty$, such that (4.2) and (4.3), and hence (4.4), hold, and also that $\sigma^{2}>0$.

(i) If $b_{k} \sim c \sqrt{t_{k}}$ as $k \rightarrow \infty$, for some $c>0$, then $\tau^{2}=\sigma^{2} / c^{2}>0$, and the bivariate process $\left(X_{y t_{k}} / \sqrt{t_{k}}, V_{y t_{k}} / t_{k}\right)_{0 \leq y \leq 1}$ converges weakly in the Skorokhod topology on $D_{0}[0,1] \times D_{0}[0,1]$ to the process $\left(\sigma \bar{W}(y), \delta_{\sigma^{2}}(y)\right)_{0 \leq y \leq 1}$, where $(W(y))_{y \geq 0}$ is an $S B M$, and $\left(\delta_{\sigma^{2}}(y)\right)_{y \geq 0}$ is a process degenerate on the line $\left\{\sigma^{2} y: y \geq 0\right\}$. This holds if $\tau^{2}>0$ in (4.3), in which case $b_{k} \sim \sigma \sqrt{t_{k}} / \tau$, as $k \rightarrow \infty$.

(ii) In particular, (4.2) and (4.3) always hold with $t_{k}$ replaced by $t, b_{k}$ replaced by $\sqrt{t}, \Lambda \equiv 0$, and $\tau^{2}=\sigma^{2}$. Thus (since we can delete the centering in (4.4) in this case), we always have that the process $\left(X_{y t} / \sqrt{t}, V_{y t} / t\right)_{0 \leq y \leq 1}$ converges weakly in the Skorokhod topology on $D_{0}[0,1] \times D_{0}[0,1]$ to the process $\left(\sigma W(y), \delta_{\sigma^{2}}(y)\right)_{0 \leq y \leq 1}$, as $t \downarrow 0$, when $\sigma^{2}>0$.

(iii) If $\tau^{2}=0$ in (4.3), then as $k \rightarrow \infty$ the process $\left(X_{y t_{k}} / b_{k}, V_{y t_{k}} / b_{k}^{2}\right)_{0 \leq y \leq 1}$ converges in probability for each $y>0$ to the process degenerate at $(0,0)$.

Remarks. (i) Proposition 4.1 via its converse, shows that if the process in (4.4) converges in distribution for the single value $y=1$, then in fact the process converges in $D_{0}[0,1]$. Similar comments apply to the other results in Section 2 . 
(ii) In [34] it is shown that the converse part of Proposition 4.1] can be strengthened, namely, when (4.2) and (4.3) hold, we have that the limit

$$
\lim _{k \rightarrow \infty} \frac{t_{k} V\left(x b_{k}\right)}{b_{k}^{2}}=\tau^{2}+\int_{|y| \leq x} y^{2} \Lambda(\mathrm{d} y)
$$

(where $x>0$ and $\pm x$ are continuity points of $\Lambda$ ) exists, rather than just (4.3).

(iii) If (4.2) and (4.3) hold in continuous time, in the sense that there is a nonstochastic function $b(t)>0, b(t) \downarrow 0$ as $t \downarrow 0$, such that (4.2) and (4.3) hold with $b_{k}$ replaced by $b\left(t_{k}\right)$ and $t_{k}$ replaced by $t$, then (4.4) holds with $t_{k}$ replaced by $t$ as $t \downarrow 0$. Also, (4.6) holds with $a_{k}$ replaced by a nonstochastic function $a(t)$. Similar comments apply to Corollary 4.2 .

(iii) The functional convergence in $D_{0}[0,1]$ in Proposition 4.1, and in the other results in Section 2. can be extended to convergence in $D_{0}[0, \infty)$ by the methods of Lindvall [29].

Proof of Proposition 4.1 and Corollary 4.2. When $y=1$ the results follow directly from Theorem 2.2 of 34 , so we need only extend them to the functional versions. These in turn follow by modifying the methods of Gikhman and Skorokhod [17, Ch. IX, Sect. 5. Thus, we prove (a), convergence of the finite-dimensional distributions of, and, (b), tightness of, the process in (4.4), as $t_{k} \downarrow 0$.

(a) Assume we have the convergence of $\left(\mathcal{I}_{t_{k}}(1), \mathcal{J}_{t_{k}}(1)\right)$, as $t_{k} \downarrow 0$, to a proper inf. div. rv, denoted $(\mathcal{I}(1), \mathcal{J}(1))$, where $\left(\mathcal{I}_{t}(y), \mathcal{J}_{t}(y)\right)$ is the process

$$
\left(\mathcal{I}_{t}(y), \mathcal{J}_{t}(y)\right):=\left(\frac{X_{y t}-y t \nu(b(t))}{b(t)}, \frac{V_{y t}}{b^{2}(t)}\right)_{0 \leq y \leq 1}
$$

indexed by $t>0$; that is, we have convergence of the process in (4.4) at $y=1$, when we set $b_{k}=b\left(t_{k}\right)$. Then it is simple to check via (2.5) that $\left(\mathcal{I}_{t_{k}}(y), \mathcal{J}_{t_{k}}(y)\right) \stackrel{\mathrm{D}}{\longrightarrow}$ $(\mathcal{I}(y), \mathcal{J}(y))$ for each fixed $y>0$. Thus, the increments of $\left(\mathcal{I}_{t_{k}}(y), \mathcal{J}_{t_{k}}(y)\right)$, that is, $\left(\mathcal{I}_{t_{k}}\left(y_{2}\right)-\mathcal{I}_{t_{k}}\left(y_{1}\right), \mathcal{J}_{t_{k}}\left(y_{2}\right)-\mathcal{J}_{t_{k}}\left(y_{1}\right)\right)$, where $0 \leq y_{1}<y_{2}$, converge in distribution to the corresponding increments of $(\mathcal{I}(y), \mathcal{J}(y))$, and from this it is easy to see that finite linear combinations of the rvs $\left(\mathcal{I}_{t_{k}}\left(y_{i}\right), \mathcal{J}_{t_{k}}\left(y_{i}\right)\right)$, for any $0 \leq y_{1}<y_{2}<\ldots<$ $y_{N}, N=2,3, \ldots$, written as linear combinations of the (independent) increments, converge in distribution to the corresponding linear combinations of $\left(\mathcal{I}\left(y_{i}\right), \mathcal{J}\left(y_{i}\right)\right)$, $i=1,2, \ldots, N$. Thus we get the required finite-dimensional convergence.

(b) For tightness, we can consider the $\mathcal{I}$ and $\mathcal{J}$ components separately, so we will restrict the discussion to $\mathcal{I}$. We need the following lemma, which generalises Lemma 1 of [17, p.480.

Lemma 4.3. Given a nontrivial Lévy process $\xi$ on $D_{0}[0, \infty)$ and a constant $b>0$, define

$$
\Gamma_{b}(\xi)=\sup _{0 \leq u<y<w \leq b} \min (|\xi(y)-\xi(u)|,|\xi(w)-\xi(y)|) .
$$

Then for any $\varepsilon>0$ we have

$$
P\left(\Gamma_{b}(\xi)>\varepsilon\right) \leq P^{2}\left(\sup _{0 \leq y \leq b}|\xi(y)| \geq \varepsilon / 2\right) .
$$

Proof of Lemma 4.3. Take $\varepsilon>0$ and suppose the event $\left\{\Gamma_{b}(\xi)>\varepsilon\right\}$ occurs. Then there is a $y \in[0, b)$ and values $u \in[0, y)$ and $w \in(y, b]$ such that

$$
|\xi(y)-\xi(u)|>\varepsilon \text { and }|\xi(w)-\xi(y)|>\varepsilon .
$$


Clearly for such a $y$ and $u \leq y$,

$$
|\xi(y)|>\varepsilon / 2 \text { or }|\xi(u)|>\varepsilon / 2 ;
$$

otherwise we would have $|\xi(y)-\xi(u)| \leq \varepsilon$, which contradicts (4.10). We also claim that for all $0 \leq s \leq y$,

$$
|\xi(y)-\xi(s)|>\varepsilon / 2 \text { or }|\xi(w)-\xi(s)|>\varepsilon / 2 .
$$

If on the contrary there existed a $0 \leq s \leq y$ such that (4.12) does not hold, then we would get $|\xi(w)-\xi(y)| \leq \varepsilon$, contradicting (4.10).

Define the passage time

$$
Y=\inf \{t>0:|\xi(t)| \geq \varepsilon / 2\}
$$

(with $\inf \phi=\infty$ ). Since $\lim \sup _{t \rightarrow \infty}|\xi(t)|=\infty$ a.s., we have $Y<\infty$ a.s., and by right continuity of $\xi$ we have $|\xi(Y)| \geq \varepsilon / 2$. Notice that in the event $\left\{\Gamma_{b}(\xi)>\varepsilon\right\}$, on account of (4.11), $Y \in(0, y]$, and thus since $\xi(0)=0$,

$$
Y \in(0, b) \text {, a.s. }
$$

Furthermore, due to (4.12), we must also have

$$
\sup _{x \in(Y, b]}|\xi(x)-\xi(Y)| \geq \varepsilon / 2 .
$$

To see this, note that, on $\{Y=y\}$ we have by (4.12) with $s=Y$ that

$$
|\xi(w)-\xi(Y)|=|\xi(w)-\xi(y)|>\varepsilon / 2,
$$

while on $\{Y<y\}$, (4.12) gives

$$
|\xi(y)-\xi(Y)|>\varepsilon / 2 \text { or }|\xi(w)-\xi(Y)|>\varepsilon / 2,
$$

with $w>Y$. Therefore in any case (4.14) is true. Hence, by (4.13) and (4.14),

$$
P\left(\Gamma_{b}(\xi)>\varepsilon\right) \leq P\left(Y \in[0, b), \sup _{x \in(Y, b]}|\xi(x)-\xi(Y)| \geq \varepsilon / 2\right) .
$$

By the strong Markov property for $\xi$, the right-hand side of (4.15) does not exceed

$$
P(Y \in[0, b]) P\left(\sup _{x \in(0, b]}|\xi(x)| \geq \varepsilon / 2\right)=P^{2}\left(\sup _{x \in(0, b]}|\xi(x)| \geq \varepsilon / 2\right),
$$

thus completing the proof of Lemma 4.3 .

Next, for reals $0<c \leq a<b \leq 1-c$, define

$$
\Delta_{a, b, c}(\xi)=\sup _{a \leq y \leq b} \sup _{y-c \leq u<y<w \leq y+c} \min (|\xi(y)-\xi(u)|,|\xi(w)-\xi(y)|) .
$$

For any integer $m>3$, let $\delta_{m}=1 / m$, and take $\varepsilon>0$. Then we see that

$$
P\left(\Delta_{\delta_{m}, 1-\delta_{m}, \delta_{m}}(\xi)>\varepsilon\right) \leq \sum_{k=1}^{m-1} P\left(\Delta_{k \delta_{m},(k+1) \delta_{m}, \delta_{m}}(\xi)>\varepsilon\right) .
$$

Observing that

$$
\Delta_{k \delta_{m},(k+1) \delta_{m}, \delta_{m}}(\xi) \stackrel{\mathrm{D}}{=} \Delta_{\delta_{m}, 2 \delta_{m}, \delta_{m}}(\xi) \text { for each } 1 \leq k \leq m-1,
$$

we get

$$
P\left(\Delta_{\delta_{m}, 1-\delta_{m}, \delta_{m}}(\xi)>\varepsilon\right) \leq(m-1) P\left(\Delta_{\delta_{m}, 2 \delta_{m}, \delta_{m}}(\xi)>\varepsilon\right) .
$$


Next,

$$
\begin{aligned}
& \Delta_{\delta_{m}, 2 \delta_{m}, \delta_{m}}(\xi)=\sup _{\delta_{m} \leq y \leq 2 \delta_{m}} \sup _{y-\delta_{m} \leq u<y<w \leq y+\delta_{m}} \min (|\xi(y)-\xi(u)|,|\xi(w)-\xi(y)|) \\
& \quad \leq \sup _{0 \leq u<y<w \leq 3 \delta_{m}} \min (|\xi(y)-\xi(u)|,|\xi(w)-\xi(y)|) .
\end{aligned}
$$

Thus, by (4.9), for all $\varepsilon>0$,

$$
P\left(\Delta_{\delta_{m}, 2 \delta_{m}, \delta_{m}}(\xi)>\varepsilon\right) \leq P\left(\Gamma_{3 \delta_{m}}(\xi)>\varepsilon\right) \leq P^{2}\left(\sup _{0 \leq y \leq 3 \delta_{m}}|\xi(y)|>\varepsilon / 2\right),
$$

which gives

$$
P\left(\Delta_{\delta_{m}, 1-\delta_{m}, \delta_{m}}(\xi)>\varepsilon\right) \leq(m-1) P^{2}\left(\sup _{0 \leq y \leq 3 \delta_{m}}|\xi(y)|>\varepsilon / 2\right) .
$$

Apply this to the process $\mathcal{I}_{t}(y)=\left(X_{y t}-y t \nu(b(t))\right) / b(t)$ defined in (4.8), using (4.1) with $b=b(t)$. The small jump process $X_{t}^{(S, b(t))}$ is a mean 0 martingale with respect to its natural filtration, with variance

$$
t\left(\sigma^{2}+\int_{|x| \leq b(t)} x^{2} \Pi(\mathrm{d} x)\right)=t V(b(t)) .
$$

Keeping $\delta_{m}=1 / m$, with $m=1,2, \ldots$, the large jump process satisfies

$$
\begin{aligned}
P\left(\sup _{0 \leq y \leq 3 \delta_{m}}\left|X_{y t}^{(B, b(t))}\right|=0\right) & \geq P\left(\text { no }\left|\Delta X_{s}\right| \text { exceeds } b(t) \text { up until time } 3 \delta_{m} t\right) \\
& =e^{-3 \delta_{m} t \bar{\Pi}(b(t))} \\
& \geq 1-3 \delta_{m} t \bar{\Pi}(b(t)), t>0 .
\end{aligned}
$$

Using these, together with Doob's inequality, (4.2), and (4.3), gives, for $\varepsilon>0$,

$$
P\left(\sup _{0 \leq y \leq 3 \delta_{m}}\left|\mathcal{I}_{t_{k}}(y)\right|>\varepsilon / 2\right) \leq \frac{96 t_{k} \delta_{m} V\left(b\left(t_{k}\right)\right)}{\varepsilon^{2} b^{2}\left(t_{k}\right)}+3 \delta_{m} t_{k} \bar{\Pi}\left(b\left(t_{k}\right)\right) \leq \frac{C \delta_{m}}{\varepsilon^{2}},
$$

for some $C>0$ and small enough $t_{k}$. Substituting for $\xi$ in (4.16) then gives

$$
\lim _{m \rightarrow \infty} \limsup _{t_{k} \downarrow 0} P\left(\Delta_{\delta_{m}, 1-\delta_{m}, \delta_{m}}(\mathcal{I})>\varepsilon\right) \leq \lim _{m \rightarrow \infty} C^{2} \delta_{m}^{2} m / \varepsilon^{4}=\lim _{m \rightarrow \infty} C^{2} \delta_{m} / \varepsilon^{4}=0 .
$$

The last is the key result in establishing tightness. It only remains, by [17], p.481, to show that

$$
\lim _{m \rightarrow \infty} \limsup _{t_{k} \downarrow 0} P\left(\sup _{0 \leq y \leq \delta_{m}}\left|\mathcal{I}_{t_{k}}(y)-\mathcal{I}_{t_{k}}(0)\right|+\sup _{1-\delta_{m} \leq y \leq 1}\left|\mathcal{I}_{t_{k}}(1)-\mathcal{I}_{t_{k}}(y)\right|>\varepsilon\right)=0
$$

for every $\varepsilon>0$. This is easily done using Doob's inequality again and completes Proposition 4.1 and Corollary 4.2 .

Proof of Theorem 2.1. If $\sigma^{2}>0$, let $b(t)=\sqrt{t}$ and $b_{k}=b\left(t_{k}\right)$, for any $t_{k} \downarrow 0$. Then (4.2) and (4.3) hold with $\Lambda \equiv 0$ and $\tau^{2}=\sigma^{2}>0$, because (see the note after (2.4)) $\lim _{x \downarrow 0} x^{2} \bar{\Pi}(x)=0$ (for any Lévy measure), $\lim _{x \downarrow 0} x \nu(x)=0$, and $\lim _{x \downarrow 0} V(x)=$ $\sigma^{2}$. These imply (2.8), and in fact (2.11) holds since $x \nu(x)=o(1)=o(V(x))$ as $x \downarrow 0$. Then Proposition 4.1 with $\tau^{2}=\sigma^{2}$ and $\Lambda_{\mathcal{I}}=\Lambda_{\mathcal{J}}=0$ gives (2.6); in fact, $\left(X_{t} / \sqrt{t}, V_{t} / t\right) \stackrel{\mathrm{D}}{\longrightarrow}\left(N\left(0, \sigma^{2}\right), \delta_{\sigma^{2}}\right)$, as $t \downarrow 0$, by Part (ii) of Corollary 4.2, Thus 
$X_{t} \in F C_{0} \subseteq F C$ at 0 . The dominated variation of $b(t), V(x)$ and $U(x)$ at 0 is obvious in this case. So we can and will assume $\sigma^{2}=0$ for the rest of the proof.

(i) Suppose $X_{t} \in F C$ at 0 , so there are $a(t), b(t)>0$, such that each $t_{k} \downarrow 0$ contains $t_{k^{\prime}} \downarrow 0$ for which (2.1) holds. Then we show that (2.8) must hold. Let

$$
R(x):=x^{2} \bar{\Pi}(x) / V(x) .
$$

Suppose in fact that (2.8) fails, so we have $\lim \sup _{x \downarrow 0} R(x)=\infty$. We first show this implies the existence of a sequence $\zeta_{k} \downarrow 0$ such that

$$
\lim _{k \rightarrow \infty} \inf _{0<\lambda_{1} \leq \lambda \leq \lambda_{2}} R\left(\lambda \zeta_{k}\right)=\infty, \text { for each } 0<\lambda_{1}<1<\lambda_{2}<\infty .
$$

To this end, fix $0<\lambda_{1}<1<\lambda_{2}<\infty$, choose $c_{n} \downarrow 0$ such that $R\left(c_{n}\right) \uparrow \infty$, let $n_{1}=\min \left\{m \geq 1: c_{m}<1 / 2\right.$, and $\left.R\left(c_{m}\right)>2^{3}\right\}$, and then for $k=1,2, \ldots$, set

$$
n_{k+1}=\min \left\{m>n_{k}: c_{m}<2^{-k / 2-1} c_{n_{k}} \text {, and } R\left(c_{m}\right)>2^{3(k+1)}\right\} .
$$

Then put $\zeta_{k}=2^{-k} c_{n_{k}}$, so that $\zeta_{k} \downarrow 0$ as $k \rightarrow \infty$. Note that $R(x) / x^{2}$ is nonincreasing on $(0, \infty)$. Choose $\lambda \in\left[1, \lambda_{2}\right]$ and $k$ such that $2^{k} \geq \lambda_{2}$. Then $\lambda \leq 2^{k}$ and

$$
\frac{R\left(\lambda \zeta_{k}\right)}{\lambda^{2}}=\frac{\zeta_{k}^{2} R\left(\lambda \zeta_{k}\right)}{\left(\lambda \zeta_{k}\right)^{2}} \geq \frac{\zeta_{k}^{2} R\left(2^{k} \zeta_{k}\right)}{\left(2^{k} \zeta_{k}\right)^{2}}=2^{-2 k} R\left(2^{k} \zeta_{k}\right)=2^{-2 k} R\left(c_{n_{k}}\right) \geq 2^{k},
$$

so $\inf _{1 \leq \lambda \leq \lambda_{2}} R\left(\lambda \zeta_{k}\right) \geq 2^{k} \rightarrow \infty$. Thus $R\left(\zeta_{k}\right) \geq 2^{k} \rightarrow \infty$, and for $\lambda \in\left[\lambda_{1}, 1\right]$, $\lambda_{1}^{2} R\left(\zeta_{k}\right) \leq \lambda^{2} R\left(\zeta_{k}\right) \leq R\left(\lambda \zeta_{k}\right)$, so $\inf _{\lambda_{1} \leq \lambda \leq 1} R\left(\lambda \zeta_{k}\right) \rightarrow \infty$. Hence (4.19) holds.

Let $t_{k}=\inf \left\{t: b(t) \geq \zeta_{k}\right\}$, so $t_{k} \downarrow 0$ and, since $b(t)$ is right continuous, $b\left(t_{k} / 2\right) \leq$ $\zeta_{k} \leq b\left(t_{k}\right)$. Now we need the following lemma.

Lemma 4.4. Suppose there is a nonstochastic function $b(t)>0$ such that every sequence $t_{k} \downarrow 0$ contains a subsequence $t_{k^{\prime}} \downarrow 0$ with

$$
\frac{X_{t_{k^{\prime}}}}{b\left(t_{k^{\prime}}\right)} \stackrel{\mathrm{D}}{\longrightarrow} Y^{\prime} \text {, as } k^{\prime} \rightarrow \infty
$$

where $Y^{\prime}$ is an a.s. finite rv, possibly degenerate, but not degenerate at 0 . Then for every $\lambda>0, b(\lambda t) \asymp b(t)$ as $t \downarrow 0$; thus there are constants $0<c_{-}(\lambda)<c_{+}(\lambda)<\infty$, for which

$$
c_{-}(\lambda) \leq \frac{b(\lambda t)}{b(t)} \leq c_{+}(\lambda), \text { once } t \leq \text { some } t_{0}(\lambda)>0 .
$$

Proof of Lemma 4.4. Assume (4.20), and, by way of contradiction, that there is a $\lambda>0$ and a sequence $t_{k} \downarrow 0$ such that $b\left(\lambda t_{k}\right) / b\left(t_{k}\right) \rightarrow 0$ or $\infty$, as $t_{k} \downarrow 0$. By (4.20), taking a further subsequence $t_{k^{\prime}}$ if necessary, we can assume that $X_{t_{k^{\prime}}} / b\left(t_{k^{\prime}}\right) \stackrel{\mathrm{D}}{\longrightarrow} Y^{\prime}$, where $Y^{\prime}$ is an a.s. finite (infinitely divisible) rv, possibly degenerate, but not degenerate at 0 . Let $y_{k^{\prime}}:=\lambda t_{k^{\prime}}$. By taking a further subsequence of $y_{k^{\prime}}$ if necessary, we can assume that $X_{y_{k^{\prime}}} / b\left(y_{k^{\prime}}\right) \stackrel{\mathrm{D}}{\longrightarrow} Y^{\prime \prime}$, where $Y^{\prime \prime}$ is an a.s. finite rv, possibly degenerate, but not degenerate at 0 . Now, because

$$
E e^{\mathrm{i} \theta X_{\lambda t} / b(t)}=\left(E e^{\mathrm{i} \theta X_{t} / b(t)}\right)^{\lambda}, \theta \in \mathbb{R}, \lambda>0, t>0,
$$

we have that $X_{\lambda t_{k^{\prime}}} / b\left(t_{k^{\prime}}\right) \stackrel{\mathrm{D}}{\longrightarrow} Y^{\prime}(\lambda)$, where $Y^{\prime}(\lambda)$ has infinitely divisible characteristic function satisfying $E e^{\mathrm{i} \theta Y^{\prime}(\lambda)}=\left(E e^{\mathrm{i} \theta Y^{\prime}}\right)^{\lambda}$, and is (finite a.s.) and not degenerate at 0 . Write

$$
\frac{X_{y_{k^{\prime}}}}{b\left(y_{k^{\prime}}\right)}=\frac{X_{\lambda t_{k^{\prime}}}}{b\left(\lambda t_{k^{\prime}}\right)}=\frac{X_{\lambda t_{k^{\prime}}}}{b\left(t_{k^{\prime}}\right)} \frac{b\left(t_{k^{\prime}}\right)}{b\left(\lambda t_{k^{\prime}}\right)}
$$


On the left, $X_{y_{k^{\prime}}} / b\left(y_{k^{\prime}}\right)$ has limit in distribution $Y^{\prime \prime}$. On the right, $X_{\lambda t_{k^{\prime}}} / b\left(t_{k^{\prime}}\right)$ has limit in distribution $Y^{\prime}(\lambda)$. $Y^{\prime}(\lambda)$ and $Y^{\prime \prime}$ are a.s. finite, and neither is degenerate at 0 . Thus $b\left(t_{k^{\prime}}\right) / b\left(\lambda t_{k^{\prime}}\right)$ cannot tend either to 0 or to $\infty$, giving a contradiction, and proving that $b(\lambda t) \asymp b(t)$ as $t \downarrow 0$.

Now to return to the proof of the theorem. Invoking the stochastic compactness, take a subsequence $t_{k^{\prime}}$ of $t_{k}$ if necessary so that (2.1) holds, where $Y^{\prime}$ is an inf. div. rv with triplet $\left(0,\left(\tau^{\prime}\right)^{2}, \Lambda^{\prime}\right)$. Then by (4.2) and (4.7), and taking a further subsequence if necessary, we have for all $x>0$ which are continuity points of the limits:

$$
t_{k^{\prime}} \bar{\Pi}\left(x b\left(t_{k^{\prime}}\right)\right) \rightarrow \bar{\Lambda}^{\prime}(x) \quad \text { and } \quad \frac{t_{k^{\prime}} V\left(x b\left(t_{k^{\prime}}\right)\right)}{b^{2}\left(t_{k^{\prime}}\right)} \rightarrow v^{\prime}(x):=\left(\tau^{\prime}\right)^{2}+\int_{0<|y| \leq x} y^{2} \Lambda^{\prime}(\mathrm{d} y) .
$$

Let $\left(\widetilde{X}_{t}\right)_{t>0}$ be a process with the same finite-dimensional distributions as, but independent of, $\left(X_{t}\right)_{t \geq 0}$, and put $X_{t}^{S}=X_{t}-\widetilde{X}_{t}$, for $t \geq 0$. Clearly, every sequence $t_{k} \downarrow 0$ contains a subsequence $t_{k^{\prime}} \downarrow 0$ such that $X_{t_{k^{\prime}}}^{S} / b\left(t_{k^{\prime}}\right)$ converges in distribution to an a.s. finite $\mathrm{rv}$ which is not degenerate at 0 (since $Y^{\prime}$ is not degenerate at any constant). The sequence $t_{k}$ was chosen so that $b\left(t_{k} / 2\right) \leq \zeta_{k} \leq b\left(t_{k}\right)$; hence by Lemma 4.4 there is a constant $c_{-}>0$ such that $c_{-} b\left(t_{k}\right) \leq \zeta_{k} \leq b\left(t_{k}\right)$ for small enough $t_{k}$. If $\Lambda^{\prime} \equiv 0$, then $\left(\tau^{\prime}\right)^{2}>0$ since $Y^{\prime}$ is nondegenerate; hence $v^{\prime}(x)>0$ for all $x>0$. Otherwise, there is an $x_{0}^{\prime}>0$, with $x_{0}^{\prime} c_{-}$a continuity point of $\bar{\Lambda}^{\prime}$, such that $v^{\prime}\left(x_{0}^{\prime} c_{-}\right) \geq \int_{|y| \leq x_{0}^{\prime} c_{-}} y^{2} \Lambda^{\prime}(\mathrm{d} y)>0$. In either case,

$$
R\left(x_{0}^{\prime} \zeta_{k^{\prime}}\right)=\frac{\left(x_{0}^{\prime} \zeta_{k^{\prime}}\right)^{2} \bar{\Pi}\left(x_{0}^{\prime} \zeta_{k^{\prime}}\right)}{V\left(x_{0}^{\prime} \zeta_{k^{\prime}}\right)} \leq\left(\frac{\left(x_{0}^{\prime}\right)^{2} b^{2}\left(t_{k^{\prime}}\right)}{t_{k^{\prime}} V\left(x_{0}^{\prime} c_{-} b\left(t_{k^{\prime}}\right)\right)}\right)\left(t_{k^{\prime}} \bar{\Pi}\left(x_{0}^{\prime} c_{-} b\left(t_{k^{\prime}}\right)\right)\right) .
$$

Now

consequently

$$
t_{k^{\prime}} \bar{\Pi}\left(x_{0}^{\prime} c_{-} b\left(t_{k^{\prime}}\right)\right) \rightarrow \bar{\Lambda}^{\prime}\left(x_{0}^{\prime} c_{-}\right)
$$

$$
\limsup _{k^{\prime} \rightarrow \infty} R\left(x_{0}^{\prime} \zeta_{k^{\prime}}\right) \leq \frac{\left(x_{0}^{\prime}\right)^{2} \bar{\Lambda}\left(x_{0}^{\prime} c_{-}\right)}{v^{\prime}\left(x_{0}^{\prime} c_{-}\right)}<\infty
$$

contradicting (4.19). Thus (2.8) holds.

Conversely, suppose (2.8) holds. Note that, after integrating by parts,

$$
U(x)=V(x)+x^{2} \bar{\Pi}(x), x>0,
$$

where $U(x)$ is defined following (2.4). So (2.8) implies the existence of a constant $c_{1} \in(0,1)$ such that

$$
\frac{x^{2} \bar{\Pi}(x)}{U(x)} \leq c_{1}<1, \text { for all small } x,
$$

$x \leq x_{0}$, say. Thus for $\lambda>1$ and $x \leq x_{0} / \lambda$ we have

$$
\frac{U(\lambda x)}{U(x)}=\exp \left(\int_{x}^{\lambda x} \frac{2 y \bar{\Pi}(y)}{U(y)} \mathrm{d} y\right) \leq e^{2 c_{1} \log \lambda}=\lambda^{2 c_{1}} .
$$

The function $U(x)$ is continuous, in fact, differentiable, at each $x>0$, with

$$
\frac{\mathrm{d}}{\mathrm{d} x}\left(\frac{U(x)}{x^{2}}\right)=\frac{-2\left(U(x)-x^{2} \bar{\Pi}(x)\right)}{x^{3}} .
$$


Further,

$$
U(x)-x^{2} \bar{\Pi}(x)=\sigma^{2}+2 \int_{0}^{x} y(\bar{\Pi}(y)-\bar{\Pi}(x)) \mathrm{d} y \geq 2 \int_{0}^{x} y(\bar{\Pi}(y)-\bar{\Pi}(x)) \mathrm{d} y .
$$

The right-hand side here could be 0 only if $\bar{\Pi}(y)$ is constant on $(0, x]$, but under our assumption $\bar{\Pi}(0+)=\infty$, this is not possible. Also in Section 1 we ensured that $\bar{\Pi}(x)>0$ for all $0<x \leq 1$, so we see that $x^{-2} U(x)$ is strictly decreasing for $x \in[0,1]$, with $x^{-2} U(x) \uparrow \infty$ as $x \downarrow 0$, while $x^{-2} U(x) \downarrow 0$ as $x \uparrow \infty$.

Now we use the following construction, which we give in slightly more generality than we need at the moment. In view of the monotonicity of $x^{-2} U(x)$ just established, for each $\lambda>0$ and $t>0$, the function

$$
b_{\lambda}(t):=\inf \left\{x>0: x^{-2} U(x) \leq(\lambda t)^{-1}\right\}
$$

is finite, positive, is such that $b_{\lambda}(t) \downarrow 0$ as $t \downarrow 0$, and is such that

$$
\frac{t U\left(b_{\lambda}(t)\right)}{b_{\lambda}^{2}(t)}=\frac{1}{\lambda}
$$

Further, $x^{-2} U(x)$, has no intervals of constancy in $[0,1]$, because of its strict monotonicity, so $b_{\lambda}(t)$ is continuous and strictly increasing in $t \in[0,1]$ for each $\lambda>0$.

For the norming function in (2.6) we will use $b(t):=b_{1}(t)$, as in (4.24) with $\lambda=1$. Take any sequence $t_{k}$, with $t_{k} \downarrow 0$ as $k \rightarrow \infty$, and let $b_{k}=b\left(t_{k}\right)$. Then for $0<x<1$,

$$
t_{k} \bar{\Pi}\left(x b_{k}\right) \leq \frac{t_{k} U\left(x b_{k}\right)}{x^{2} b_{k}^{2}} \leq \frac{t_{k} U\left(b_{k}\right)}{x^{2} b_{k}^{2}}=\frac{1}{x^{2}},
$$

since $U(x) \geq x^{2} \bar{\Pi}(x), x>0$. Also, by (4.23), for $x>1$ and $k$ so large that $x b_{k} \leq x_{0}$, we have

$$
t_{k} \bar{\Pi}\left(x b_{k}\right) \leq \frac{t_{k} U\left(x b_{k}\right)}{x^{2} b_{k}^{2}} \leq \frac{x^{2 c_{1}-2} t_{k} U\left(b_{k}\right)}{b_{k}^{2}}=x^{2 c_{1}-2},
$$

where $c_{1}<1$ does not depend on the choice of subsequence. It follows that $\lim \sup _{t_{k} \downarrow 0} t_{k}\left(\bar{\Pi}\left(x b_{k}\right)+U\left(x b_{k}\right) / b_{k}^{2}\right)<\infty$ for all $x>0$. By Helly's theorem we can take a subsequence $t_{k^{\prime}}$ of $t_{k}$ so that, as $k^{\prime} \rightarrow \infty$,

$$
t_{k^{\prime}} \bar{\Pi}^{ \pm}\left(x b_{k^{\prime}}\right) \rightarrow \bar{\Lambda}^{ \pm}(x) \quad \text { and } \quad \frac{t_{k^{\prime}} U\left(x b_{k^{\prime}}\right)}{b_{k^{\prime}}^{2}} \rightarrow u^{\prime}(x)
$$

at continuity points of the limits, where $\bar{\Lambda}^{\prime \pm}(x)$ are finite nonincreasing functions, and $u^{\prime}(x)$ is a finite nondecreasing function, on $(0, \infty)$. Then $\bar{\Lambda}^{\prime}(x):=\bar{\Lambda}^{\prime+}(x)+$ $\bar{\Lambda}^{\prime-}(x) \leq x^{2 c_{1}-2}$ for all $x>1$, so $\bar{\Lambda}^{\prime}(+\infty)=0$. Also, for $0<x<1$, by Fatou's lemma,

$$
u^{\prime}(x)=\lim _{k^{\prime} \rightarrow \infty} t_{k^{\prime}} b_{k^{\prime}}^{-2} U\left(b_{k^{\prime}}\right)=\lim _{k^{\prime} \rightarrow \infty} 2 t_{k^{\prime}} \int_{0}^{x} y \bar{\Pi}\left(y b_{k^{\prime}}\right) \mathrm{d} y \geq 2 \int_{0}^{x} y \bar{\Lambda}^{\prime}(y) \mathrm{d} y
$$

(recall $\left.\sigma^{2}=0\right)$, so we have $\int_{(0, \infty)}\left(y^{2} \wedge 1\right)\left|\mathrm{d} \bar{\Lambda}^{\prime}(y)\right|<\infty$. Thus $\bar{\Lambda}^{\prime \pm}$ are the tails of a Lévy measure, $\Lambda^{\prime}$. Further, since $b(t) \equiv b_{1}(t)$ as defined in (4.24), for any $x>0$ we have

$$
u^{\prime}(x)=\lim _{k^{\prime} \rightarrow \infty} \frac{t_{k^{\prime}} U\left(x b_{k^{\prime}}\right)}{b_{k^{\prime}}^{2}}=1+2 \lim _{k^{\prime} \rightarrow \infty} t_{k^{\prime}} \int_{1}^{x} y \bar{\Pi}\left(y b_{k^{\prime}}\right) \mathrm{d} y=1+2 \int_{1}^{x} y \bar{\Lambda}^{\prime}(y) \mathrm{d} y,
$$


by dominated convergence. Consequently, by (4.26), after an integration by parts,

$$
\lim _{k^{\prime} \rightarrow \infty} \frac{t_{k^{\prime}} V\left(x b_{k^{\prime}}\right)}{b_{k^{\prime}}^{2}}=\left(\tau^{\prime}\right)^{2}+\int_{0<|y| \leq x} y^{2} \Lambda^{\prime}(\mathrm{d} y)
$$

where $\left(\tau^{\prime}\right)^{2}:=1-2 \int_{0}^{1} y \bar{\Lambda}^{\prime}(y) \mathrm{d} y$. Note that $\left(\tau^{\prime}\right)^{2} \geq 0$ as we see by letting $x \downarrow 0$ in (4.27). We have (4.2) and (4.3) for the subsequence $t_{k^{\prime}}$, so Proposition 4.1 gives that $\left(X_{t_{k^{\prime}}}-a\left(t_{k^{\prime}}\right)\right) / b_{k^{\prime}} \stackrel{\mathrm{D}}{\longrightarrow} \mathcal{I}^{\prime}$ as $k^{\prime} \rightarrow \infty$, where $\mathcal{I}^{\prime}$ is an inf. div. rv with triplet $\left(0,\left(\tau^{\prime}\right)^{2}, \Lambda^{\prime}\right)$. If $\mathcal{I}^{\prime}$ is degenerate it must be degenerate at 0 , in which case $\tau^{\prime}=0 \equiv \Lambda^{\prime}$. But then $\left(\tau^{\prime}\right)^{2}=1$, a contradiction. Hence all subsequential limits are nondegenerate, and so $X \in F C$ at 0 .

Next we show the joint convergence, i.e., that (2.6) holds when $X \in F C$. Assume each $t_{k} \downarrow 0$ contains $t_{k^{\prime}} \downarrow 0$ for which (2.1) holds with $Y^{\prime}$ finite and nondegenerate. By Proposition 4.1, this implies the joint convergence in (2.6), where $a(t)$ can be chosen as $t \nu(b(t))$, and $\mathcal{I}^{\prime}$ is nondegenerate. Also, $P\left(\left(\tau^{\prime}\right)^{2}+\mathcal{J}^{\prime} \leq 0\right)=0$, as follows. By Proposition 4.1 we know that $\left(\tau^{\prime}\right)^{2}+\mathcal{J}^{\prime}$ is inf. div. with triplet $\left(\left(\tau^{\prime}\right)^{2}, 0, \Lambda_{\mathcal{J}^{\prime}}\right)$, where $\bar{\Lambda}_{\mathcal{J}^{\prime}}(x)=\bar{\Lambda}^{\prime}(\sqrt{x})$ for $x>0$. A similar argument to 4.23) shows that $U(x \lambda) / U(x) \geq \lambda^{2 c_{1}}$ for $\lambda<1, x \leq x_{0}$. Thus, with $b(t) \equiv b_{1}(t)$ as defined in (4.24),

$$
\frac{t U(\lambda b(t))}{b^{2}(t)} \geq \lambda^{2 c_{1}} \frac{t U(b(t))}{b^{2}(t)}=\lambda^{2 c_{1}}, \text { for } 0<\lambda<1, b(t) \leq x_{0} .
$$

From (4.26) we get, for $\lambda>0$,

$$
u^{\prime}(\lambda)=\lim _{k^{\prime} \rightarrow \infty} \frac{t_{k^{\prime}} U\left(\lambda b_{k^{\prime}}\right)}{b_{k^{\prime}}^{2}}=\left(\tau^{\prime}\right)^{2}+2 \int_{0}^{\lambda} y \bar{\Lambda}^{\prime}(y) \mathrm{d} y
$$

and so

$$
\left(\tau^{\prime}\right)^{2}+\lambda^{2} \bar{\Lambda}^{\prime}(0+) \geq\left(\tau^{\prime}\right)^{2}+2 \int_{0}^{\lambda} y \bar{\Lambda}^{\prime}(y) \mathrm{d} y \geq \lambda^{2 c_{1}} .
$$

Since $c_{1}<1$, letting $\lambda \downarrow 0$ here gives $\tau^{\prime}>0$ or $\bar{\Lambda}^{\prime}(0+)=\infty$. By Lemma 4.3 in [34, this implies $P\left(\left(\tau^{\prime}\right)^{2}+\mathcal{J}^{\prime}=0\right)=0$.

That $b(t)$ has the continuity and monotonicity properties claimed follows from our choice of $b(t)=b_{1}(t)$, as described following (4.24). Note that $b(t)$ can also be defined as the (unique) functional inverse of the strictly decreasing function $f(x):=x^{-2} U(x), x>0$, evaluated at $x=1 / t$. The dominated variation condition established using (4.23) can be written as $\lim \sup _{x \downarrow 0} U(\lambda x) / U(x) \leq \lambda^{2-\alpha}$, for $\lambda>1$, with $\alpha=2-2 c_{1} \in(0,2]$. This implies a corresponding condition on the inverse of $f(\cdot)$, giving rise to the inequality $\limsup _{t \downarrow 0} b(\lambda t) / b(t) \leq \lambda^{1 / \alpha}$, for $\lambda>1$. That $a(t)=t \nu(b(t))+O(b(t))$ follows from (4.6), because every $t_{k} \downarrow 0$ contains a $t_{k^{\prime}} \downarrow 0$ such that $a\left(t_{k^{\prime}}\right)=t_{k^{\prime}} \nu\left(b\left(t_{k^{\prime}}\right)\right)+\left(\beta^{\prime}+o(1)\right) b\left(t_{k^{\prime}}\right)$ for some $\beta^{\prime} \in(-\infty, \infty)$.

Now $x^{2} \bar{\Pi}(x) \leq c_{1} U(x)=c_{1}\left(x^{2} \bar{\Pi}(x)+V(x)\right)$ gives $x^{2} \bar{\Pi}(x) \leq c_{2} V(x)$, where $c_{2}:=c_{1} /\left(1-c_{1}\right)$, so

$$
V(\lambda x) \leq U(\lambda x) \leq \lambda^{2-\alpha} U(x) \leq\left(1+c_{2}\right) \lambda^{2-\alpha} V(x)=\lambda^{2-\alpha} V(x) /\left(1-c_{1}\right)
$$

for $\lambda>1$ and $x \leq x_{0}$. That $\bar{\Lambda}^{\prime}(x) \leq x^{2-\alpha}$ for $x>1$ follows from (4.25).

Finally, we prove that (2.6) is equivalent to (2.7). Of course (2.6) implies (2.7), so let (2.7) hold; thus, each $t_{k} \downarrow 0$ contains $t_{k^{\prime}} \downarrow 0$ for which $V_{t_{k^{\prime}}} / b_{k^{\prime}}^{2} \stackrel{\mathrm{D}}{\longrightarrow} \mathcal{K}^{\prime}$, as $k^{\prime} \rightarrow \infty$, where $b_{k}:=b\left(t_{k}\right)$, and $P\left(\mathcal{K}^{\prime} \leq 0\right)=0$. We will prove that (4.2) and (4.3), in fact, (4.7), hold through the subsequence. $\left(V_{t}\right)_{t \geq 0}$ is itself a Lévy process, so by 
Lemma 4.1 of 34, $\mathcal{K}^{\prime}$ is inf. div., and $b_{k^{\prime}} \rightarrow 0$ as $k^{\prime} \rightarrow \infty$. Take $\sigma^{2}=0, \theta_{1}=0$ and $\theta_{2}=\theta / b_{k^{\prime}}^{2}$ in (2.5) to get

$$
\begin{aligned}
& E e^{\mathrm{i} \theta V_{k_{k^{\prime}}} / b_{k^{\prime}}^{2}}=\exp \left(t_{k^{\prime}} \int_{(-\infty, \infty)}\left(e^{\mathrm{i} \theta x^{2} / b_{k^{\prime}}^{2}}-1\right) \Pi(\mathrm{d} x)\right) \\
& =\exp \left(t_{k^{\prime}} \int_{(0, \infty)}\left(e^{\mathrm{i} \theta y}-1\right) \Pi_{V}\left(b_{k^{\prime}} \mathrm{d} y\right)\right),
\end{aligned}
$$

where $\Pi_{V}$ satisfies $\bar{\Pi}_{V}(y):=\bar{\Pi}(\sqrt{y})$ for each $y>0$. We are told that the right-hand side of (4.28) converges, as $k^{\prime} \rightarrow \infty$, to the c.f. of an inf. div. rv, $\mathcal{K}^{\prime}$, which is concentrated on $(0, \infty)$, a.s., thus, is of the form

$$
\exp \left(\mathrm{i} \theta\left(\tau^{\prime}\right)^{2}+\int_{(0, \infty)}\left(e^{\mathrm{i} \theta y}-1\right) \Lambda_{\mathcal{J}^{\prime}}(\mathrm{d} y)\right)
$$

where $\left(\tau^{\prime}\right)^{2} \geq 0$ and $\Lambda_{\mathcal{J}^{\prime}}$ is a measure on $(0, \infty)$ such that $\int_{(0, \infty)}(x \wedge 1) \Lambda_{\mathcal{J}^{\prime}}(\mathrm{d} x)<\infty$ (see Feller, 16], p.571). Using criteria for the convergence of inf. div. distributions (Kallenberg, 27, Thm. 15.14, Part (ii), p.295), this means that, as $k^{\prime} \rightarrow \infty$, for each $h>0$,

$$
a_{k^{\prime}}^{h}:=t_{k^{\prime}} \int_{0<|y| \leq h} y \Pi_{V}\left(b_{k^{\prime}}^{2} \mathrm{~d} y\right) \rightarrow a^{h}:=\left(\tau^{\prime}\right)^{2}+\int_{0<|y| \leq h} y \Lambda_{\mathcal{J}^{\prime}}(\mathrm{d} y),
$$

and

$$
t_{k^{\prime}} \bar{\Pi}_{V}\left(b_{k^{\prime}}^{2} h\right) \rightarrow \bar{\Lambda}_{\mathcal{J}^{\prime}}(h),
$$

vaguely, on $(0, \infty)$. Now, define $\bar{\Lambda}(h)=\bar{\Lambda}_{\mathcal{J}^{\prime}}\left(h^{2}\right)$, for $h>0$. This is the tail of a Lévy measure, $\Lambda$, and (4.29) gives

$$
t_{k^{\prime}} b_{k^{\prime}}^{-2} \int_{0<|y| \leq h b_{k^{\prime}}} y^{2} \Pi(\mathrm{d} y) \rightarrow\left(\tau^{\prime}\right)^{2}+\int_{0<|y| \leq h} y^{2} \Lambda^{\prime}(\mathrm{d} y),
$$

while by (4.30), taking a further subsequence if necessary, we can ensure that

$$
t_{k^{\prime}} \bar{\Pi}^{ \pm}\left(b_{k^{\prime}} h\right)^{\prime} \rightarrow \bar{\Lambda}^{ \pm}(h),
$$

at continuity points. These imply (4.2) and (4.7), hence (4.3); thus we have (2.1), and $X_{t} \in F C$ at 0 . Note that $V$ has no Gaussian component, so $\mathcal{K}^{\prime}$ has triplet $\left(\left(\tau^{\prime}\right)^{2}, 0, \Lambda_{\mathcal{J}^{\prime}}\right)$.

To summarize, we have shown that (i) $\Longrightarrow$ (iv), (iv) $\Longrightarrow$ (i), (i) $\Longrightarrow$ (ii), (ii) $\Longrightarrow$ (iii) and (iii) $\Longrightarrow$ (i), completing the proof of Theorem 2.1.

Proof of Theorem 2.2. Let (2.8) hold. Then by Part (i) of Theorem 2.1] we have the convergence in (2.1), so (4.2) and (4.3) hold along a subsequence by the converse part of Proposition 4.1. Hence the process in (4.4) converges along the subsequence to the limit specified in Theorem 2.2

Proof of Theorem 2.3. Now we prove the results concerning $F C_{0}$. As shown at the start of the proof of Theorem 2.1, we can assume $\sigma^{2}=0$. Assume (2.11), so (2.8) holds, and so, as shown in the proof of Theorem 2.1, there is a function $b(t)>0$ such that every sequence $t_{k} \downarrow 0$ contains a subsequence $t_{k^{\prime}} \downarrow 0$ for which (2.6) holds, in 
which we can choose $b(t)$ to satisfy (4.24) with $\lambda=1$, and $a(t)=t \nu(b(t))+O(b(t))$. (2.11) also implies that

$$
\limsup _{k^{\prime} \rightarrow \infty} \frac{t_{k^{\prime}}\left|\nu\left(b_{k^{\prime}}\right)\right|}{b_{k^{\prime}}}=\limsup _{k^{\prime} \rightarrow \infty} \frac{b_{k^{\prime}}\left|\nu\left(b_{k^{\prime}}\right)\right|}{U\left(b_{k^{\prime}}\right)}<\infty
$$

so, taking a further subsequence if necessary, we can make $a\left(b_{k^{\prime}}\right) / b_{k^{\prime}} \rightarrow \beta^{\prime}$, for some $\beta^{\prime} \in \mathbb{R}$. Then (2.6) gives $X_{t_{k^{\prime}}} / b_{k^{\prime}} \stackrel{\mathrm{D}}{\longrightarrow} \mathcal{I}^{\prime}+\beta^{\prime}$, as $k^{\prime} \rightarrow 0$, and $\mathcal{I}^{\prime}+\beta^{\prime}$ is a nondegenerate inf. div. rv with triplet $\left(\beta^{\prime},\left(\tau^{\prime}\right)^{2}, \Lambda^{\prime}\right)$. Thus (2.10) holds, for the case $y=1$, so $X_{t} \in F C_{0}$. The functional version of (2.10) follows from Proposition 4.1 .

Conversely, suppose $X \in F C_{0}$, so there is a function $b(t)>0$ such that $X_{t} / b(t)$ is stochastically compact as $t \downarrow 0$, but that 2.11) fails. By Theorem 2.1 with $a(t)=0$ we have (2.8), so it must be the case that

$$
\limsup _{x \downarrow 0} \frac{x|\nu(x)|}{V(x)}=\infty .
$$

Choose $x_{k} \downarrow 0$ such that $\lim _{x_{k} \downarrow 0} x_{k}\left|\nu\left(x_{k}\right)\right| / V\left(x_{k}\right)=\infty$, and define $t_{k} \downarrow 0$ by $t_{k}=$ $\inf \left\{t>0: b(t) \geq x_{k}\right\}$. Then, as shown in the proof of Theorem 2.1. $c_{-} b\left(t_{k}\right) \leq$ $x_{k} \leq b\left(t_{k}\right)$ for some $c_{-}>0$ and all small $t_{k}$. Invoking the stochastic compactness of $X_{t} / b(t)$, we can find a subsequence $t_{k^{\prime}} \downarrow 0$ for which $X_{t_{k^{\prime}}} / b_{k^{\prime}} \stackrel{\mathrm{D}}{\longrightarrow} Y^{\prime}$, as $k^{\prime} \rightarrow \infty$, where $Y^{\prime}$ is finite and nondegenerate, and $b_{k^{\prime}}=b\left(t_{k^{\prime}}\right)$. Then by (4.2), (4.6) and (4.7), we have, for $x>0$ such that $\pm x$ are continuity points of $\Lambda^{\prime}$,

$$
\begin{aligned}
& \lim _{k^{\prime} \rightarrow \infty} t_{k^{\prime}} \bar{\Pi}\left(x b_{k^{\prime}}\right)=\bar{\Lambda}^{\prime}(x), \\
& \lim _{k^{\prime} \rightarrow \infty} \frac{t_{k^{\prime}} V\left(x b_{k^{\prime}}\right)}{b_{k^{\prime}}^{2}}=\left(\tau^{\prime}\right)^{2}+\int_{|y| \leq x} y^{2} \Lambda^{\prime}(\mathrm{d} y)
\end{aligned}
$$

and

$$
\lim _{k^{\prime} \rightarrow \infty} \frac{t_{k^{\prime}} \nu\left(x b_{k^{\prime}}\right)}{b_{k^{\prime}}}=\beta^{\prime}+\int_{x<|y| \leq 1} y \Lambda^{\prime}(\mathrm{d} y)
$$

for some $\tau^{\prime} \geq 0, \beta^{\prime} \in \mathbb{R}$, and Lévy measure $\Lambda^{\prime}$. Since $c_{-} b_{k^{\prime}} \leq x_{k^{\prime}} \leq b_{k^{\prime}}$ for all large $k^{\prime}$, this means that $t_{k^{\prime}}\left|\nu\left(x_{k^{\prime}}\right)\right|=O\left(b_{k^{\prime}}\right)$ as $k^{\prime} \rightarrow \infty$. Thus, if $\tau^{\prime}>0$, then as $k^{\prime} \rightarrow \infty$,

$$
\frac{t_{k^{\prime}}\left|\nu\left(x_{k^{\prime}}\right)\right|}{b_{k^{\prime}}} \geq \frac{\left(\left(\tau^{\prime}\right)^{2}+o(1)\right) b_{k^{\prime}}\left|\nu\left(x_{k^{\prime}}\right)\right|}{V\left(c_{-} b_{k^{\prime}}\right)} \geq \frac{\left(\left(\tau^{\prime}\right)^{2}+o(1)\right) x_{k^{\prime}}\left|\nu\left(x_{k^{\prime}}\right)\right|}{V\left(x_{k^{\prime}}\right)} \rightarrow \infty,
$$

giving a contradiction. If $\tau^{\prime}=0$, then $\bar{\Lambda}^{\prime}(0+)=\infty$, as shown in Theorem 2.1, so $\Lambda^{\prime}$ has points of increase arbitrarily near 0 . Thus $\int_{|y| \leq 1} y^{2} \Lambda^{\prime}(\mathrm{d} y)>0$, and a similar argument to (4.31) again gives a contradiction. Hence (2.11) holds.

Proof of Theorem 2.4. If $\sigma^{2}>0$, we have $X_{t} / \sqrt{t} \stackrel{\mathrm{D}}{\longrightarrow} N\left(0, \sigma^{2}\right)$ and $V_{t} / t \stackrel{\mathrm{P}}{\longrightarrow} \sigma^{2}$ as shown at the start of the proof of Theorem 2.1 so we have $X \in D(N)$ and $X \in$ $D_{0}(N)$, while $\lim _{x \downarrow 0} x^{2} \bar{\Pi}(x)=0, \lim _{x \downarrow 0} V(x)=\sigma^{2}$ and $x \nu(x)=o(1)=o(V(x))$, as $x \downarrow 0$, and hence all of (i)-(vii) hold. So we can and will assume throughout the proof that $\sigma^{2}=0$.

The equivalence of (i) and (ii), and of (v) and (vi), is in Theorem 2.5 of [11. Further, the proof of that theorem shows that $b(t)$ may be chosen according to (4.24) with $\lambda=1$, so it is continuous and strictly increasing, and further, shows that $U(x)$ and hence $V(x)$ are slowly varying at 0 . Thus the function $f(x):=x^{-2} U(x), x>0$, 
is in $R V(-2)$ at 0 (recall that this means, is regularly varying with index -2 at 0 ); hence the inverse function $f^{\leftarrow}(x) \in R V(-1 / 2)$ at 0 (see [5], Prop. 1.5.15, p.29). This means that $b(t)=f^{\leftarrow}(1 / t) \in R V(1 / 2)$ at 0 , as claimed.

Clearly (v) implies (i), and next we show that (i) implies (vi), hence (v). Assume (i), so (2.13) holds; thus $V(x) \sim U(x)$ and $U \in S V$ at 0 . Now, as $x \downarrow 0$,

$$
\frac{x|\nu(x)|}{U(x)}=\frac{x\left|\gamma-\int_{x<|y| \leq 1} y \Pi(\mathrm{d} y)\right|}{U(x)}=\frac{x\left|\int_{x<|y| \leq 1} y \Pi(\mathrm{d} y)\right|}{U(x)}+o(1),
$$

because $U \in S V$ at 0 means $\lim _{x \downarrow 0} U(x) / x=\infty$. So consider

$$
x \int_{x<|y| \leq 1}|y| \Pi(\mathrm{d} y)=x \int_{x<y \leq 1} y|\mathrm{~d} \bar{\Pi}(y)| \leq x^{2} \bar{\Pi}(x)+x \int_{x}^{1} \bar{\Pi}(y) \mathrm{d} y,
$$

in which $x^{2} \bar{\Pi}(x)=o(U(x))$ by (2.13). So we look at

$$
\begin{aligned}
2 x \int_{x}^{1} \bar{\Pi}(y) \mathrm{d} y & =x \int_{x}^{1} y^{-1} \mathrm{~d} U(y) \\
& =x \int_{x}^{1} y^{-2} U(y) \mathrm{d} y-U(x)+x U(1) \\
& =x \int_{x}^{1} y^{-2}(U(y)-U(x)) \mathrm{d} y-x(U(x)-U(1)) \\
& =\int_{1}^{1 / x} y^{-2}(U(x y)-U(x)) \mathrm{d} y+o(U(x)) .
\end{aligned}
$$

It will complete the proof to show that the last integral is $o(U(x))$ as $x \downarrow 0$. This is done as follows. Since $U \in S V$ at 0 , there is a uniform bound of the form: given $A>1$ and $\varepsilon \in(0,1)$, there exists $x_{0}=x_{0}(A, \varepsilon) \in(0,1)$, such that for all $0<x \leq x_{0}$ and $y \geq 1$,

$$
1 \leq \frac{U(x y)}{U(x)} \leq A y^{\varepsilon}
$$

(see [5], p.25). Thus for all $0<x<z<x_{0} \leq 1$ we have

$$
\begin{aligned}
\int_{1}^{1 / x}\left(\frac{U(x y)}{U(x)}-1\right) \frac{\mathrm{d} y}{y^{2}} & \leq \int_{1}^{1 / z}\left(\frac{U(x y)}{U(x)}-1\right) \frac{\mathrm{d} y}{y^{2}}+A \int_{1 / z}^{1 / x} \frac{\mathrm{d} y}{y^{2-\varepsilon}} \\
& \leq\left(\frac{U(x / z)}{U(x)}-1\right)+A \frac{z^{1-\varepsilon}}{1-\varepsilon} .
\end{aligned}
$$

Let $x \downarrow 0$. Then the first term on the right-hand side tends to 0 , by the slow variation of $U(x)$ at 0 . Then let $z \downarrow 0$. The second term tends to 0 because $\varepsilon<1$. Hence the last integral in (4.32) is $o(U(x))$ as $x \downarrow 0$, and we have shown that (2.16) holds.

(v) implies (iv), and (iv) implies (iii), clearly, and next we prove that (iii) implies (i). Let (iii) hold. Take any sequence $t_{k} \downarrow 0$ and choose $t_{k^{\prime}} \downarrow 0$ so that $\left(X_{t_{k^{\prime}}}-\right.$ $a\left(t_{k^{\prime}}\right) / b\left(t_{k^{\prime}}\right) \stackrel{\mathrm{D}}{\longrightarrow} N\left(0,\left(\tau^{\prime}\right)^{2}\right)$, where $\tau^{\prime}>0$. Proposition 4.1 (more precisely, the remark following it) gives

$$
t_{k^{\prime}} \bar{\Pi}^{ \pm}\left(\lambda b\left(t_{k^{\prime}}\right)\right) \rightarrow 0 \text { and } \frac{t_{k^{\prime}} V\left(\lambda b\left(t_{k^{\prime}}\right)\right)}{b^{2}\left(t_{k^{\prime}}\right)} \rightarrow\left(\tau^{\prime}\right)^{2}
$$

thus $U\left(\lambda b\left(t_{k^{\prime}}\right)\right) / U\left(b\left(t_{k^{\prime}}\right)\right) \rightarrow 1$, for all $\lambda>0$, as $k^{\prime} \rightarrow \infty$. Consequently we have $U(\lambda b(t)) / U(b(t)) \rightarrow 1$ for all $\lambda>0$, as $t \downarrow 0$. Given $x>0$, let $t=t(x)=\inf \{y>0$ : 
$b(y) \geq x\}$. Then, using Lemma 4.4, $c_{-} b(t) \leq b(t / 2) \leq x \leq b(t)$, for some $c_{-}>0$, for all small $x$. Hence

$$
\frac{U\left(\lambda c_{-} b(t)\right)}{U(b(t))} \leq \frac{U(\lambda x)}{U(x)} \leq \frac{U(\lambda b(t))}{U(b(t))} \frac{U(b(t))}{U\left(c_{-} b(t)\right)}
$$

shows that $U(\lambda x) / U(x) \rightarrow 1$ for all $\lambda>0$, as $x \downarrow 0$. Thus $U \in S V$ at 0 . Then

$$
0=\lim _{t \downarrow 0}\left(\frac{U(2 x)}{U(x)}-1\right) \geq \limsup _{t \downarrow 0} \frac{3 x^{2} \bar{\Pi}(2 x)}{U(x)},
$$

together with $U \in S V$ as $t \downarrow 0$, shows that (2.13) holds.

It remains only to prove the equivalence of (vii) with the rest. Let (2.12) hold with $\tau>0$. Then by Proposition 4.1, taken in continuous time (cf. Remark (iii) following Corollary 4.2), we have $V_{t} / b^{2}(t) \stackrel{\mathrm{P}}{\longrightarrow} \tau^{2} \in(0, \infty)$, as $t \downarrow 0$, so (vii) holds.

Conversely, let (vii) hold with the given $b(t)$, take a sequence $t_{k} \downarrow 0$, and choose a subsequence $t_{k^{\prime}} \downarrow 0$ with $V_{t_{k^{\prime}}} / b^{2}\left(t_{k^{\prime}}\right) \stackrel{\mathrm{P}}{\longrightarrow}\left(\tau^{\prime}\right)^{2}$, as $k^{\prime} \rightarrow \infty$, where $\tau^{\prime} \in(0, \infty)$. Now by using the representation of the c.f. in (2.5), we see that $V_{t}$ has canonical triplet $\left(\gamma_{V}, 0, \Pi_{V}\right)$, where $\gamma_{V}:=\int_{|x| \leq 1} x^{2} \Pi(\mathrm{d} x)$, and (cf. (4.28) $), \bar{\Pi}_{V}(x)=\bar{\Pi}(\sqrt{x}), x>0$. In this case

$$
\nu_{V}(x):=\gamma_{V}-\int_{x<y \leq 1} y \Pi_{V}(\mathrm{~d} y)=\int_{0<|y| \leq x} y^{2} \Pi(\mathrm{d} y)=V(x), x>0
$$

(recall $\sigma^{2}=0$ ), so we can apply (4.2) and (4.6) to get, for $x>0$, as $k^{\prime} \rightarrow \infty$,

$$
t_{k^{\prime}} \bar{\Pi}_{V}\left(x b^{2}\left(t_{k^{\prime}}\right)\right)=t_{k^{\prime}} \bar{\Pi}\left(\sqrt{x} b\left(t_{k^{\prime}}\right)\right) \rightarrow 0
$$

and

$$
\frac{t_{k^{\prime}} \nu_{V}\left(x b^{2}\left(t_{k^{\prime}}\right)\right)}{b^{2}\left(t_{k^{\prime}}\right)}=\frac{t_{k^{\prime}} V\left(\sqrt{x} b^{2}\left(t_{k^{\prime}}\right)\right)}{b^{2}\left(t_{k^{\prime}}\right)} \rightarrow\left(\tau^{\prime}\right)^{2} .
$$

Thus by Proposition 4.1. $\left(X_{t_{k^{\prime}}}-t_{k^{\prime}} \nu\left(b\left(t_{k^{\prime}}\right)\right)\right) / b\left(t_{k^{\prime}}\right) \stackrel{\mathrm{D}}{\longrightarrow} N\left(0,\left(\tau^{\prime}\right)^{2}\right)$. Consequently, (2.14) holds, so $X_{t} \in D(N)$.

Remark. The same argument as in Part (iv) of Theorem 2.4 shows that we may omit the centering term for $X$ in Theorem 2.1 provided the index $\alpha$ defined in Part (iv) of Theorem 2.1 is in $(1,2]$. In other words, (2.8) is equivalent to (2.11) when $U(x \lambda) / U(x)=O\left(\lambda^{2-\alpha}\right)$, as $x \downarrow 0$, for each $\lambda>1$, provided $\alpha \in(1,2]$.

\section{Proofs for Section 3}

We first set out some preliminary results, then prove Theorems 3.1 3.6. Note at first that the big jump component of $X$ cannot contribute to the weak convergence behavior at 0 , in the following sense. Let $\tau$ be the (exponentially distributed) time of the first jump of $X_{t}$ exceeding 1 in modulus. Using (4.1) with $b=1$ (and $\nu(1)=\gamma)$, the inequality

$$
P\left(\sum_{0<s \leq t}\left|\Delta X_{s}\right| \mathbf{1}_{\left\{\left|\Delta X_{s}\right|>1\right\}}>0\right) \leq P(\tau \leq t),
$$

together with $\lim _{t \downarrow 0} P(\tau \leq t)=0$, shows that jumps larger than 1 in modulus can be ignored when discussing the weak convergence behavior of $X_{t}$ at 0 . Consequently, throughout the remainder of the paper, we can and will assume that $\Pi(\cdot)$ is 
supported on $[-1,1]$. Recall that we always assume $\bar{\Pi}(0+)=\infty$; thus, in addition, we have $\bar{\Pi}(1)=0$, but $\bar{\Pi}(x)>0$ for $x \in(0,1)$, throughout.

We continue to use (4.1) with $b=1$. Since the big jump component is absent, we can write $X_{t}=$ a.s. $\lim _{\varepsilon \downarrow 0} X_{t}(\varepsilon)$, where for $t>0, \varepsilon \in(0,1)$,

$$
\begin{aligned}
X_{t}(\varepsilon) & =\sigma Z_{t}+t \gamma+\sum_{0<s \leq t} \Delta X_{s} \mathbf{1}_{\left\{\varepsilon<\left|\Delta X_{s}\right| \leq 1\right\}}-t \int_{\varepsilon<|x| \leq 1} x \Pi(\mathrm{d} x) \\
& =\sigma Z_{t}+t \nu(\varepsilon)+\sum_{i=1}^{N_{t}(\varepsilon)} J_{i}(\varepsilon),
\end{aligned}
$$

for $\left(J_{i}(\varepsilon)\right)_{i=1,2, \ldots}$ i.i.d. and distributed as $\Pi(\mathrm{d} x) \mathbf{1}_{\{\varepsilon<|x| \leq 1\}} / \bar{\Pi}(\varepsilon)$, and $\left(N_{t}(\varepsilon)\right)_{t \geq 0}$ independently distributed as a Poisson process with rate $\bar{\Pi}(\varepsilon)$.

Also define, for $t>0, \varepsilon \in(0,1)$,

$$
V_{t}(\varepsilon):=\sigma^{2} t+\sum_{0<s \leq t}\left(\Delta X_{s}\right)^{2} \mathbf{1}_{\left\{\varepsilon<\left|\Delta X_{s}\right| \leq 1\right\}}=\sigma^{2} t+\sum_{i=1}^{N_{t}(\varepsilon)} J_{i}^{2}(\varepsilon),
$$

so $V_{t}=$ a.s. $\lim _{\varepsilon \downarrow 0} V_{t}(\varepsilon)$.

Recall the definition of $b_{\lambda}(t)$ in (4.24). We will deduce Theorem 3.1 from the following analogue of Theorem 2 of [21], and Corollary 5.2 is immediate from it.

Proposition 5.1. There is a nonstochastic function a $(t)$ such that

$$
\frac{X_{t}-a(t)}{\sqrt{V_{t}}} \text { is relatively compact as } t \downarrow 0
$$

if and only if

$$
\limsup _{t \downarrow 0} \frac{\left|t \nu\left(b_{\lambda}(t)\right)-a(t)\right|}{b_{\lambda}(t)}<\infty,
$$

for all small, and hence, all, $\lambda>0$.

Corollary 5.2 (Corollary to Proposition 5.1). (i) $\left(X_{t}-t \nu\left(b_{\lambda}(t)\right) / \sqrt{V_{t}}\right.$ is always relatively compact as $t \downarrow 0$, for any $\lambda>0$.

(ii) If $X_{t}$ is symmetric, then $X_{t} / \sqrt{V_{t}}$ is always relatively compact as $t \downarrow 0$.

Proof of Proposition [5.1. Fix $\lambda>0$ and define $b_{\lambda}(t)$ by (4.24).

First suppose $\sigma^{2}>0$. From (4.24) we see that $b_{\lambda}(t) \sim \sigma \sqrt{\lambda t}$ as $t \downarrow 0$. From Part (ii) of Corollary 4.2, we have $X_{t} / \sqrt{\sigma^{2} t} \stackrel{\mathrm{D}}{\longrightarrow} N(0,1)$ and $V_{t} / t \stackrel{\mathrm{P}}{\longrightarrow} \sigma^{2}$, as $t \downarrow 0$. So $\left(X_{t}-a(t)\right) / \sqrt{V_{t}}$ is relatively compact as $t \downarrow 0$ if and only if $a(t)=O(\sqrt{t})$, as $t \downarrow 0$. Likewise, $t \nu(t)=o(1)$ as $t \downarrow 0$, so $t\left|\nu\left(b_{\lambda}(t)\right)\right| / b_{\lambda}(t)=o\left(t / b_{\lambda}^{2}(t)\right)=o(1)$, and (5.4) holds if and only if $a(t)=O\left(b_{\lambda}(t)\right)=O(\sqrt{t})$ as $t \downarrow 0$. So Proposition 5.1 is true when $\sigma^{2}>0$.

Next suppose $\sigma^{2}=0$, and take $\lambda>0, t>0$ small enough for $b_{\lambda}(t)<1$, and $\varepsilon \in\left(0, b_{\lambda}(t)\right)$. We further decompose $X_{t}(\varepsilon)$ in (5.1) as

$$
X_{t}(\varepsilon)=t \nu(\varepsilon)+T_{t}(\varepsilon, \lambda)+R_{t}(\varepsilon, \lambda),
$$

where

$$
T_{t}(\varepsilon, \lambda):=\sum_{i=1}^{N_{t}(\varepsilon)} J_{i}(\varepsilon) \mathbf{1}_{\left\{\left|J_{i}(\varepsilon)\right| \leq b_{\lambda}(t)\right\}}
$$


and

$$
R_{t}(\varepsilon, \lambda):=\sum_{i=1}^{N_{t}(\varepsilon)} J_{i}(\varepsilon) \mathbf{1}_{\left\{b_{\lambda}(t)<\left|J_{i}(\varepsilon)\right| \leq 1\right\}}=\sum_{i=1}^{N_{t}(\varepsilon)} J_{i}(\varepsilon) \mathbf{1}_{\left\{\left|J_{i}(\varepsilon)\right|>b_{\lambda}(t)\right\}}
$$

(the last equality holding since $\bar{\Pi}(1)=0)$. Then we can calculate

$$
E\left(T_{t}(\varepsilon, \lambda)\right)=t \bar{\Pi}(\varepsilon) \int_{\varepsilon<|x| \leq b_{\lambda}(t)} x \Pi(\mathrm{d} x) / \bar{\Pi}(\varepsilon)=t \int_{\varepsilon<|x| \leq b_{\lambda}(t)} x \Pi(\mathrm{d} x)=: \alpha_{t}(\varepsilon, \lambda)
$$

and

$$
\operatorname{Var}\left(T_{t}(\varepsilon, \lambda)\right) \leq t \int_{\varepsilon<|x| \leq b_{\lambda}(t)} x^{2} \Pi(\mathrm{d} x)
$$

Note that

$$
\begin{aligned}
t \nu(\varepsilon)+\alpha_{t}(\varepsilon, \lambda) & =t\left(\gamma-\int_{\varepsilon<|x| \leq 1} x \Pi(\mathrm{d} x)+\int_{\varepsilon<|x| \leq b_{\lambda}(t)} x \Pi(\mathrm{d} x)\right) \\
& =t\left(\gamma-\int_{b_{\lambda}(t)<|x| \leq 1} x \Pi(\mathrm{d} x)\right)=t \nu\left(b_{\lambda}(t)\right) .
\end{aligned}
$$

We can thus write, for any $L>0$,

$$
\begin{aligned}
& P\left(\left|t \nu\left(b_{\lambda}(t)\right)-a(t)\right|>3 L^{2} b_{\lambda}(t)\right)=P\left(\left|t \nu(\varepsilon)+\alpha_{t}(\varepsilon, \lambda)-a(t)\right|>3 L^{2} b_{\lambda}(t)\right) \\
& \leq P\left(\left|T_{t}(\varepsilon, \lambda)-\alpha_{t}(\varepsilon, \lambda)\right|>L^{2} b_{\lambda}(t)\right)+P\left(\left|t \nu(\varepsilon)+T_{t}(\varepsilon, \lambda)-a(t)\right|>2 L^{2} b_{\lambda}(t)\right),
\end{aligned}
$$

and we proceed by estimating the quantities on the right-hand side.

By Chebychev's inequality, for any $L>0, K>0$,

$$
\begin{aligned}
P\left(\left|T_{t}(\varepsilon, \lambda)-\alpha_{t}(\varepsilon, \lambda)\right|>L K b_{\lambda}(t)\right) & \leq \frac{\operatorname{Var}\left(T_{t}(\varepsilon, \lambda)\right)}{L^{2} K^{2} b_{\lambda}^{2}(t)} \leq \frac{t \int_{\varepsilon<|x| \leq b_{\lambda}(t)} x^{2} \Pi(\mathrm{d} x)}{L^{2} K^{2} b_{\lambda}^{2}(t)} \\
& \leq \frac{t U\left(b_{\lambda}(t)\right)}{L^{2} K^{2} b_{\lambda}^{2}(t)}=\frac{1}{L^{2} K^{2} \lambda} .
\end{aligned}
$$

This gives a bound for the first term on the right-hand side of (5.8). The second term on the right-hand side of (5.8) does not exceed

$$
P\left(\left|t \nu(\varepsilon)+T_{t}(\varepsilon, \lambda)-a(t)\right|>2 L^{2} b_{\lambda}(t), L b_{\lambda}(t) \geq \sqrt{V_{t}(\varepsilon)}\right)+P\left(L b_{\lambda}(t)<\sqrt{V_{t}(\varepsilon)}\right) .
$$

Let

$$
U_{t}(\varepsilon, \lambda):=\sum_{i=1}^{N_{t}(\varepsilon)}\left(J_{i}^{2}(\varepsilon) \wedge b_{\lambda}^{2}(t)\right) .
$$

It's not hard to check that $E\left(J_{1}^{2}(\varepsilon) \wedge b_{\lambda}^{2}(t)\right)=\varepsilon^{2}+\left(U\left(b_{\lambda}(t)\right)-U(\varepsilon)\right) / \bar{\Pi}(\varepsilon)$, so we can use Wald's lemma to obtain

$$
E\left(U_{t}(\varepsilon, \lambda)\right)=\lambda^{-1} b_{\lambda}^{2}(t)-t V(\varepsilon) .
$$


In the event $\left\{\max _{1 \leq i \leq N_{t}(\varepsilon)}\left|J_{i}(\varepsilon)\right| \leq b_{\lambda}(t)\right\}$ we have $V_{t}(\varepsilon)=U_{t}(\varepsilon, \lambda)$, so

$$
\begin{aligned}
& P\left(\sqrt{V_{t}(\varepsilon)}>L b_{\lambda}(t)\right) \leq P\left(V_{t}(\varepsilon)>L^{2} b_{\lambda}^{2}(t), \max _{1 \leq i \leq N_{t}(\varepsilon)}\left|J_{i}(\varepsilon)\right| \leq b_{\lambda}(t)\right) \\
& +1-P\left(\max _{1 \leq i \leq N_{t}(\varepsilon)}\left|J_{i}(\varepsilon)\right| \leq b_{\lambda}(t)\right) \\
\leq & P\left(U_{t}(\varepsilon, \lambda)>L^{2} b_{\lambda}^{2}(t)\right)+1-\sum_{n \geq 0} P^{n}\left(\left|J_{1}(\varepsilon)\right| \leq b_{\lambda}(t)\right) P\left(N_{t}(\varepsilon)=n\right) \\
\leq & \frac{E\left(U_{t}(\varepsilon, \lambda)\right)}{L^{2} b_{\lambda}^{2}(t)}+1-\sum_{n \geq 0} e^{-t \bar{\Pi}(\varepsilon)}\left(t \bar{\Pi}(\varepsilon) P\left(\left|J_{1}(\varepsilon)\right| \leq b_{\lambda}(t)\right)\right)^{n} / n ! \\
= & \frac{\left(\lambda^{-1} b_{\lambda}^{2}(t)-t V(\varepsilon)\right)}{L^{2} b_{\lambda}^{2}(t)}+1-e^{-t \bar{\Pi}(\varepsilon) P\left(\left|J_{1}(\varepsilon)\right|>b_{\lambda}(t)\right)} \\
\leq & \lambda^{-1} L^{-2}+1-e^{-t \bar{\Pi}\left(b_{\lambda}(t)\right)} \leq \lambda^{-1} L^{-2}+1-e^{-\lambda^{-1}} .
\end{aligned}
$$

(In the last inequality, recall that $x^{2} \bar{\Pi}(x) \leq U(x)$, so $t \bar{\Pi}\left(b_{\lambda}(t)\right) \leq 1 / \lambda$.) This is our bound for the second term on the right-hand side of (5.10).

Next, to estimate $R_{t}(\varepsilon, \lambda)$ in (5.6), put

$$
S_{t}(\varepsilon, \lambda):=\sum_{i=1}^{N_{t}(\varepsilon)} \mathbf{1}_{\left\{b_{\lambda}(t)<\left|J_{i}(\varepsilon)\right| \leq 1\right\}}=\sum_{i=1}^{N_{t}(\varepsilon)} \mathbf{1}_{\left\{\left|J_{i}(\varepsilon)\right|>b_{\lambda}(t)\right\}} .
$$

Then by Cauchy-Schwarz,

$$
\left|R_{t}(\varepsilon, \lambda)\right|^{2} \leq\left(\sum_{i=1}^{N_{t}(\varepsilon)} J_{i}^{2}(\varepsilon)\right)\left(\sum_{i=1}^{N_{t}(\varepsilon)} \mathbf{1}_{\left\{b_{\lambda}(t)<\left|J_{i}(\varepsilon)\right| \leq 1\right\}}\right)=V_{t}(\varepsilon) S_{t}(\varepsilon, \lambda) .
$$

Thus, for $L>0$,

$$
\begin{aligned}
P\left(\left|R_{t}(\varepsilon, \lambda)\right|>L \sqrt{V_{t}(\varepsilon)}\right) & \leq P\left(S_{t}(\varepsilon, \lambda)>L^{2}\right) \leq L^{-2} E\left(S_{t}(\varepsilon, \lambda)\right) \\
& =L^{-2}(t \bar{\Pi}(\varepsilon)) \bar{\Pi}\left(b_{\lambda}(t)\right) / \bar{\Pi}(\varepsilon) \leq L^{-2} \lambda^{-1} .
\end{aligned}
$$

So (cf. (5.5)) we see that the first term on the right-hand side of (5.10) does not exceed

$$
\begin{aligned}
& P\left(\left|t \nu(\varepsilon)+T_{t}(\varepsilon, \lambda)-a(t)\right|>2 L \sqrt{V_{t}(\varepsilon)}\right) \\
\leq & P\left(\left|X_{t}(\varepsilon)-a(t)\right|>L \sqrt{V_{t}(\varepsilon)}\right)+P\left(\left|R_{t}(\varepsilon, \lambda)\right|>L \sqrt{V_{t}(\varepsilon)}\right) \\
\leq & P\left(\left|X_{t}(\varepsilon)-a(t)\right|>L \sqrt{V_{t}(\varepsilon)}\right)+L^{-2} \lambda^{-1},
\end{aligned}
$$

by (5.14). Going back to (5.8), we put together (5.9) with $K=L$, and the bounds in (5.13) and (5.10), to deduce that

$$
\begin{aligned}
& P\left(\left|t \nu\left(b_{\lambda}(t)\right)-a(t)\right|>3 L^{2} b_{\lambda}(t)\right)=\mathbf{1}_{\left\{\left|t \nu\left(b_{\lambda}(t)\right)-a(t)\right|>3 L^{2} b_{\lambda}(t)\right\}} \\
\leq & \lambda^{-1} L^{-4}+\lambda^{-1} L^{-2}+1-e^{-\lambda^{-1}}+P\left(\left|X_{t}(\varepsilon)-a(t)\right|>L \sqrt{V_{t}(\varepsilon)}\right)+L^{-2} \lambda^{-1} .
\end{aligned}
$$


As $\varepsilon \downarrow 0, X_{t}(\varepsilon) \rightarrow X_{t}$ and $V_{t}(\varepsilon) \rightarrow V_{t}$, a.s. Choose $L$ so large that $\lambda^{-1} L^{-4}+$ $2 L^{-2} \lambda^{-1}<e^{-\lambda^{-1}} / 2$. Then, letting $\varepsilon \downarrow 0$, gives

$$
\mathbf{1}_{\left\{\left|t \nu\left(b_{\lambda}(t)\right)-a(t)\right|>3 L^{2} b_{\lambda}(t)\right\}} \leq P\left(\left|X_{t}-a(t)\right|>L \sqrt{V_{t}}\right)+1-e^{-\lambda^{-1}} / 2 .
$$

Now assume (5.3), i.e., that $\left|X_{t}-a(t)\right| / \sqrt{V_{t}}$ is relatively compact. Letting $t \downarrow 0$ then $L \rightarrow \infty$ gives

$$
\lim _{L \rightarrow \infty} \limsup _{t \downarrow 0} \mathbf{1}_{\left\{\left|t \nu\left(b_{\lambda}(t)\right)-a(t)\right|>3 L^{2} b_{\lambda}(t)\right\}} \leq 1-e^{-\lambda^{-1}} / 2<1 .
$$

So, for $L \geq$ some $L_{0}(\lambda)>0$, and $t \leq$ some $t_{0}(L, \lambda)>0$, the indicator function $\mathbf{1}_{\left\{\left|t \nu\left(b_{\lambda}(t)\right)-a(t)\right|>3 L^{2} b_{\lambda}(t)\right\}}$ is strictly less than 1 ; hence we have $\left|t \nu\left(b_{\lambda}(t)\right)-a(t)\right| \leq$ $3 L^{2} b_{\lambda}(t)$. Thus for $t \leq t_{0}\left(L_{0}, \lambda\right)$,

$$
\frac{\left|t \nu\left(b_{\lambda}(t)\right)-a(t)\right|}{b_{\lambda}(t)} \leq 3 L_{0}^{2}
$$

which implies (5.4).

For the converse, suppose (5.4) holds for all sufficiently small $\lambda>0$. Fix $\lambda \in$ $(0,1)$ so small that

$$
c(\lambda):=\sup _{0<t \leq \lambda} \frac{\left|t \nu\left(b_{\lambda}(t)\right)-a(t)\right|}{b_{\lambda}(t)}<\infty .
$$

Then for $t>0, \varepsilon>0$, and $L>0$,

$$
\begin{aligned}
& P\left(\left|X_{t}(\varepsilon)-a(t)\right|>3 L \sqrt{V_{t}(\varepsilon)}\right) \\
\leq & P\left(\left|X_{t}(\varepsilon)-t \nu\left(b_{\lambda}(t)\right)\right|>3 L \sqrt{V_{t}(\varepsilon)}-c(\lambda) b_{\lambda}(t)\right) \\
\leq & P\left(\left|X_{t}(\varepsilon)-t \nu\left(b_{\lambda}(t)\right)\right|>2 L \sqrt{V_{t}(\varepsilon)}\right)+P\left(L \sqrt{V_{t}(\varepsilon)} \leq c(\lambda) b_{\lambda}(t)\right) .
\end{aligned}
$$

To deal with the first term on the right-hand side, take $K \in\left(0, \lambda^{-1 / 2}\right)$, and suppose $\varepsilon>0$ is chosen so small that $t V(\varepsilon)<\left(\lambda^{-1}-K^{2}\right) b_{\lambda}^{2}(t)$. Recall that $t \nu\left(b_{\lambda}(t)\right)=$ $t \nu(\varepsilon)+\alpha_{t}(\varepsilon, \lambda)$, by (5.7), while $X_{t}(\varepsilon)=t \nu(\varepsilon)+T_{t}(\varepsilon, \lambda)+R_{t}(\varepsilon, \lambda)$ by (5.5), and argue as follows:

$$
\begin{aligned}
& P\left(\left|X_{t}(\varepsilon)-t \nu(\varepsilon)-\alpha_{t}(\varepsilon, \lambda)\right|>2 L \sqrt{V_{t}(\varepsilon)}\right) \\
\leq & P\left(\left|T_{t}(\varepsilon, \lambda)-\alpha_{t}(\varepsilon, \lambda)\right|>L \sqrt{V_{t}(\varepsilon)}\right)+P\left(\left|R_{t}(\varepsilon, \lambda)\right|>L \sqrt{V_{t}(\varepsilon)}\right) \\
\leq & P\left(\left|T_{t}(\varepsilon, \lambda)-\alpha_{t}(\varepsilon, \lambda)\right|>L K b_{\lambda}(t)\right)+P\left(\sqrt{V_{t}(\varepsilon)} \leq K b_{\lambda}(t)\right) \\
& +P\left(\left|R_{t}(\varepsilon, \lambda)\right|>L \sqrt{V_{t}(\varepsilon)}\right) \\
\leq & \left.\frac{1}{\lambda L^{2} K^{2}}+P\left(\sqrt{V_{t}(\varepsilon)} \leq K b_{\lambda}(t)\right)+\frac{1}{\lambda L^{2}} \text { (by (15.9) and (5.14) }\right) .
\end{aligned}
$$

Now $V_{t}(\varepsilon) \geq U_{t}(\varepsilon, \lambda)$, so for $t>0$,

$$
P\left(\sqrt{V_{t}(\varepsilon)}>K b_{\lambda}(t)\right) \geq P\left(U_{t}(\varepsilon, \lambda)>K^{2} b_{\lambda}^{2}(t)\right) .
$$


Using a second moment version of Wald's lemma we see that

$$
\begin{aligned}
\operatorname{Var}\left(U_{t}(\varepsilon, \lambda)\right) & \leq t \bar{\Pi}(\varepsilon) E\left(J_{1}^{4}(\varepsilon) \wedge b_{\lambda}^{4}(t)\right) \\
& =t b_{\lambda}^{4}(t) \bar{\Pi}\left(b_{\lambda}(t)\right)+t \int_{\varepsilon \leq|y| \leq b_{\lambda}(t)} y^{4} \Pi(d y) \\
& \leq t b_{\lambda}^{2}(t)\left(b_{\lambda}^{2}(t) \bar{\Pi}\left(b_{\lambda}(t)\right)+\int_{\varepsilon \leq|y| \leq b_{\lambda}(t)} y^{2} \Pi(d y)\right) \\
& \leq t b_{\lambda}^{2}(t) U\left(b_{\lambda}(t)\right)=\lambda^{-1} b_{\lambda}^{4}(t) .
\end{aligned}
$$

Recall that we keep $K^{2}<\lambda^{-1}$ and $t V(\varepsilon)<\left(\lambda^{-1}-K^{2}\right) b_{\lambda}^{2}(t)$. There is a onesided Chebychev inequality of the form $P(Y-E Y<x) \geq x^{2} /\left(x^{2}+\operatorname{Var} Y\right)$, for any rv $Y$ and $x>0$ (e.g., [2, p.70). Apply this with $Y=-U_{t}(\varepsilon, \lambda)$, recalling that $E\left(U_{t}(\varepsilon, \lambda)\right)=\lambda^{-1} b_{\lambda}^{2}(t)-t V(\varepsilon)$, by (5.12), to get

$$
\begin{aligned}
P\left(\sqrt{V_{t}(\varepsilon)}>K b_{\lambda}(t)\right) & \geq P\left(U_{t}(\varepsilon, \lambda)>K^{2} b_{\lambda}^{2}(t)\right) \\
& =P\left(-U_{t}(\varepsilon, \lambda)+E\left(U_{t}(\varepsilon, \lambda)\right)<\left(\lambda^{-1}-K^{2}\right) b_{\lambda}^{2}(t)-t V(\varepsilon)\right) \\
& \geq \frac{\left(\left(\lambda^{-1}-K^{2}\right) b_{\lambda}^{2}(t)-t V(\varepsilon)\right)^{2}}{\left(\left(\lambda^{-1}-K^{2}\right) b_{\lambda}^{2}(t)-t V(\varepsilon)\right)^{2}+\operatorname{Var}\left(U_{t}(\varepsilon, \lambda)\right)} \\
& \geq \frac{\left(1-K^{2} \lambda\right)^{2} b_{\lambda}^{4}(t)-2 t \lambda\left(1-K^{2} \lambda\right) b_{\lambda}^{2}(t) V(\varepsilon)}{b_{\lambda}^{4}(t)(1+\lambda)+t^{2} \lambda^{2} V^{2}(\varepsilon)} .
\end{aligned}
$$

For the second term on the right-hand side of (5.15), use (5.17) with $K$ replaced by $K_{\lambda}:=c(\lambda) L^{-1}$, with $L>c(\lambda) \sqrt{\lambda}$, so $K_{\lambda}<\lambda^{-1 / 2}$. Thus, finally,

$$
\begin{gathered}
P\left(\left|X_{t}(\varepsilon)-a(t)\right|>3 L \sqrt{V_{t}(\varepsilon)}\right) \\
\leq \frac{1}{\lambda L^{2} K^{2}}+\left[1-\frac{\left(1-K^{2} \lambda\right)^{2} b_{\lambda}^{4}(t)-2 t \lambda\left(1-K^{2} \lambda\right) b_{\lambda}^{2}(t) V(\varepsilon)}{b_{\lambda}^{4}(t)(1+\lambda)+t^{2} \lambda^{2} V^{2}(\varepsilon)}\right] \\
+\left[1-\frac{\left(1-K_{\lambda}^{2} \lambda\right)^{2} b_{\lambda}^{4}(t)-2 t \lambda\left(1-K_{\lambda}^{2} \lambda\right) b_{\lambda}^{2}(t) V(\varepsilon)}{b_{\lambda}^{4}(t)(1+\lambda)+t^{2} \lambda^{2} V^{2}(\varepsilon)}\right]+\frac{1}{\lambda L^{2}} .
\end{gathered}
$$

Let $\varepsilon \downarrow 0$, recalling that $X_{t}(\varepsilon) \rightarrow X_{t}$ and $V_{t}(\varepsilon) \rightarrow V_{t}$, a.s. Then let $t \downarrow 0$, then let $L \rightarrow \infty$, noting that $K_{\lambda}=c(\lambda) L^{-1} \rightarrow 0$, and then let $K \downarrow 0$, to see that

$$
\lim _{L \rightarrow \infty} \limsup _{t \downarrow 0} P\left(\left|X_{t}-a(t)\right|>3 L \sqrt{V_{t}}\right) \leq \frac{2 \lambda}{1+\lambda} .
$$

Then let $\lambda \downarrow 0$ to get (5.3).

Proof of Theorem 3.1. Recall that we have $\bar{\Pi}(1)=0$ and $\bar{\Pi}(0+)=\infty$. Suppose $X_{t} / \sqrt{V_{t}}$ is relatively compact but there is a sequence $x_{k} \downarrow 0$ such that

$$
\frac{x_{k}\left|\nu\left(x_{k}\right)\right|}{U\left(x_{k}\right)} \rightarrow \infty, \text { as } k \rightarrow \infty .
$$

Let $t_{k}=x_{k}^{2} / U\left(x_{k}\right)$, so $x_{k}=b_{1}\left(t_{k}\right)$, in the notation of (4.24). Then

$$
\frac{t_{k}\left|\nu\left(b_{1}\left(t_{k}\right)\right)\right|}{b_{1}\left(t_{k}\right)}=\frac{t_{k}\left|\nu\left(x_{k}\right)\right|}{x_{k}}=\frac{x_{k}\left|\nu\left(x_{k}\right)\right|}{U\left(x_{k}\right)} \rightarrow \infty
$$

which contradicts (5.4) with $a(t)=0$ and $\lambda=1$. 
Conversely, suppose $\lim \sup _{x \downarrow 0} x|\nu(x)| / U(x)<c<\infty$. Then with $a(t) \equiv 0$ we have

$$
\limsup _{t \downarrow 0} \frac{\left|a(t)-t \nu\left(b_{\lambda}(t)\right)\right|}{b_{\lambda}(t)}=\limsup _{t \downarrow 0} \frac{b_{\lambda}(t)\left|\nu\left(b_{\lambda}(t)\right)\right|}{\lambda U\left(b_{\lambda}(t)\right)} \leq \frac{c}{\lambda}
$$

so (5.4) holds with $a(t)=0$, and $X_{t} / \sqrt{V_{t}}$ is relatively compact as $t \downarrow 0$, by Proposition 5.1

Proof of Theorem 3.2. Assume $X_{t}$ is symmetric. We show that $Y_{t}=X_{t} / \sqrt{V_{t}}$ is stochastically compact, at 0 . If $\sigma^{2}>0$, then $Y_{t} \stackrel{\mathrm{D}}{\longrightarrow} N(0,1)$ as $t \downarrow 0$, so we can assume $\sigma^{2}=0$.

As observed in Section 3, $Y_{t}$ is always relatively compact when $X_{t}$ is symmetric. Take $t_{k} \downarrow 0$ such that $Y_{t_{k}} \stackrel{\mathrm{D}}{\longrightarrow} Y$, where $Y$ is finite a.s. From Proposition 5.1 of [34] we have the representation

$$
Y_{t} \stackrel{\mathrm{D}}{=} \frac{\sum_{i=1}^{\infty} s_{i} \varphi\left(S_{i} / 2 t\right)}{\sqrt{\sum_{i=1}^{\infty} \varphi^{2}\left(S_{i} / 2 t\right)}}
$$

where

$$
S_{i}=\sum_{j=1}^{i} \varpi_{j} i=1,2, \ldots,
$$

and $\varpi_{1}, \varpi_{2}, \ldots$ is a sequence of i.i.d. exponential random variables with mean 1 ; also, $s_{1}, s_{2}, \ldots$ is a sequence of i.i.d. random signs, independent of $\varpi_{1}, \varpi_{2}, \ldots$, with $P\left\{s_{1}=1\right\}=P\left\{s_{1}=-1\right\}=1 / 2$, and, further,

$$
\varphi(s)=\sup \{y: \bar{\Pi}(y)>s\}, s>0,
$$

where the supremum of the empty set is taken as 0 . (5.18) shows that $Y_{t}$ is symmetrically distributed, so if $Y$ is degenerate at a constant $y$, then $y=0$, and $Y_{t_{k}} \stackrel{\mathrm{P}}{\longrightarrow} 0$. As also outlined in [34] (see p.444), $E Y_{t}=0, E Y_{t}^{2}=1$ and $E Y_{t}^{4} \leq 3$. Now $Y_{t_{k}} \stackrel{\mathrm{P}}{\longrightarrow} 0$ implies by uniform integrability that $E Y_{t_{k}}^{2} \rightarrow 0$, a contradiction. So all subsequential limits of $Y_{t}$ as $t \downarrow 0$ are nondegenerate; thus $Y_{t}$ is stochastically compact.

Proof of Theorem 3.3. If $\sigma^{2}>0$, then $X_{t} / \sqrt{V_{t}} \stackrel{\mathrm{D}}{\longrightarrow} N(0,1)$, hence is stochastically compact. So suppose $\sigma^{2}=0$. Let $X_{t} \in F C_{0}$ at 0 . Then by (2.10) there is a nonstochastic function $b(t)$, such that each sequence $t_{k} \downarrow 0$ contains a subsequence $t_{k^{\prime}} \downarrow 0$ for which

$$
\left(\frac{X_{t_{k^{\prime}}}}{b\left(t_{k^{\prime}}\right)}, \frac{V_{t_{k^{\prime}}}}{b^{2}\left(t_{k^{\prime}}\right)}\right) \stackrel{\mathrm{D}}{\longrightarrow}\left(\beta^{\prime}+\tau^{\prime} Z(1)+\mathcal{I}^{\prime}(1),\left(\tau^{\prime}\right)^{2}+\mathcal{J}^{\prime}(1)\right),
$$

for some constants $\beta^{\prime} \in \mathbb{R}$ and $\left(\tau^{\prime}\right)^{2} \geq 0$, with $Z(1)$ a standard normal rv, independent of $\left(\mathcal{I}^{\prime}(1), \mathcal{J}^{\prime}(1)\right)$. Further, $\mathcal{I}^{\prime}(1)$ is a nondegenerate inf. div. rv with triplet $\left(0,0, \Lambda^{\prime}(\mathrm{d} x)\right)$, and

$$
P\left(\left(\tau^{\prime}\right)^{2}+\mathcal{J}^{\prime}(1) \leq 0\right)=0
$$

We claim that

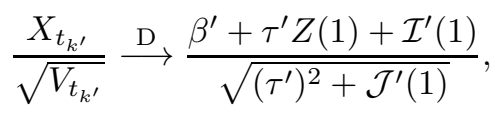


a finite rv, a.s. To see this, set

$$
\left(\beta^{\prime}+\tau^{\prime} Z(1)+\mathcal{I}(1)^{\prime},\left(\tau^{\prime}\right)^{2}+\mathcal{J}(1)^{\prime}\right)=(W, S) .
$$

Notice that for each $\varepsilon>0$, by continuity of the function $g_{\varepsilon}(x, y)=x / \sqrt{|y| \vee \varepsilon}$ on $\mathbb{R}^{2}$, we have

$$
g_{\varepsilon}\left(X_{t_{k^{\prime}}} / b\left(t_{k^{\prime}}\right), V_{t_{k^{\prime}}} / b^{2}\left(t_{k^{\prime}}\right)\right) \stackrel{\mathrm{D}}{\longrightarrow} g_{\varepsilon}(W, S) .
$$

Observe that (5.19) and (5.20) imply that

$$
\begin{gathered}
\lim _{\varepsilon \downarrow 0} \limsup _{k \rightarrow \infty} P\left(g_{\varepsilon}\left(X_{t_{k^{\prime}}} / b\left(t_{k^{\prime}}\right), V_{t_{k^{\prime}}} / b^{2}\left(t_{k^{\prime}}\right) \leq \varepsilon\right)\right) \\
\leq \lim _{\varepsilon \downarrow 0} \limsup _{k \rightarrow \infty} P\left(\left|V_{t_{k^{\prime}}} / b^{2}\left(t_{k^{\prime}}\right)\right| \leq \varepsilon\right)=0 .
\end{gathered}
$$

Furthermore, from (5.20) we get

$$
\lim _{\varepsilon \downarrow 0} P\left(W / \sqrt{S} \neq g_{\varepsilon}(W, \sqrt{S})\right) \leq \lim _{\varepsilon \downarrow 0} P\{S \leq \varepsilon\}=0 .
$$

Using (5.22), (5.23) and (5.24) we can readily verify that (5.21) holds.

Finally, apply the argument on pp.364-365 of Giné and Mason [19] to see that the distribution of any such subsequential limit rv cannot be concentrated on a finite number of points, in particular, cannot be degenerate.

Before proving Theorem 3.4, we need some preliminaries. Let $\widetilde{\Delta}_{t}^{(1)}$ be the jump $\Delta X_{s}$ of largest modulus in $(0, t]$. More, precisely, recall the definition of $X_{t}(\varepsilon)$, $N_{t}(\varepsilon)$, and the $J_{i}(\varepsilon)$ in (5.1). Let $J_{N_{t}(\varepsilon)}^{(1)}(\varepsilon)$ be the $J_{i}(\varepsilon)$ such that

$$
\max _{1 \leq k \leq i-1}\left|J_{k}(\varepsilon)\right|<\left|J_{i}(\varepsilon)\right| \geq \max _{i+1 \leq k \leq N_{t}(\varepsilon)}\left|J_{k}(\varepsilon)\right|
$$

(if there is such an $i$; set $J_{N_{t}(\varepsilon)}^{(1)}(\varepsilon)=J_{1}(\varepsilon)$ if $\left|J_{1}(\varepsilon)\right| \geq \max _{2 \leq k \leq N_{t}(\varepsilon)}\left|J_{k}(\varepsilon)\right|$, and $J_{N_{t}(\varepsilon)}^{(1)}(\varepsilon)=J_{N_{t}(\varepsilon)}(\varepsilon)$ if $\left.\left|J_{N_{t}(\varepsilon)}(\varepsilon)\right|>\max _{1 \leq k<N_{t}(\varepsilon)}\left|J_{k}(\varepsilon)\right|\right)$. Then set

$$
\widetilde{\Delta}_{t}^{(1)}=\text { a.s. } \lim _{\varepsilon \downarrow 0} J_{N_{t}(\varepsilon)}^{(1)}(\varepsilon) .
$$

Notice that this limit exists. In fact, once $\varepsilon$ is small enough for $N_{t}(\varepsilon) \geq 1$, then $J_{N_{t}(\varepsilon)}^{(1)}(\varepsilon)$ is well defined, and does not depend on $\varepsilon$. For definiteness, we have selected the $J_{N_{t}(\varepsilon)}^{(1)}(\varepsilon)$ which occurs first, if there is more than one.

Lemma 5.3. Assume $\sigma^{2}=0$ and suppose $\bar{\Pi}(x) \in S V$ at 0 . Then $X$ is of bounded variation. Assume further that it has drift $\delta=0$. Then we have

$$
\frac{X_{t}}{\widetilde{\Delta}_{t}^{(1)}} \stackrel{\mathrm{P}}{\longrightarrow} 1 \text { and } \frac{V_{t}}{\left(\widetilde{\Delta}_{t}^{(1)}\right)^{2}} \stackrel{\mathrm{P}}{\longrightarrow} 1 \text {, as } t \downarrow 0 .
$$

If in addition $X$ is symmetric, then $Y_{t}=X_{t} / \sqrt{V_{t}} \stackrel{\mathrm{D}}{\longrightarrow} Y$, where $Y= \pm 1$, each with probability $1 / 2$.

Proof of Lemma 5.3. Assume that $\sigma^{2}=0$ and that $\bar{\Pi}(x) \in S V$ at 0 . Then $\int_{0}^{1} \bar{\Pi}(x) \mathrm{d} x<\infty$, so $X$ is of bounded variation with drift $\delta$, say. By assumption, $\delta=0$. Write ${ }^{(1)} \widetilde{X}_{t}:=X_{t}-\widetilde{\Delta}_{t}^{(1)}$. It suffices to show that ${ }^{(1)} \widetilde{X}_{t} / \widetilde{\Delta}_{t}^{(1)} \stackrel{\mathrm{P}}{\longrightarrow} 0$, as $t \downarrow 0$. 
We will use (5.1), in which $\sigma^{2}=0$, and the centering term $\gamma-\int_{\varepsilon<|x| \leq 1} x \Pi(\mathrm{d} x) \rightarrow$ $\delta=0$ as $\varepsilon \downarrow 0$. So we can write $X_{t}=$ a.s. $\lim _{\varepsilon \downarrow 0} X_{t}(\varepsilon)$, where

$$
X_{t}(\varepsilon):=\sum_{i=1}^{N_{t}(\varepsilon)} J_{i}(\varepsilon)
$$

and the $J_{i}(\varepsilon)$ and $N_{t}(\varepsilon)$ have the properties listed after (5.1).

On the event $\left\{N_{t}(\varepsilon)=n\right\}$, set

$$
R_{n}(\varepsilon):=\frac{\sum_{i=1}^{n}\left|J_{i}(\varepsilon)\right|-\left|J_{n}^{(1)}(\varepsilon)\right|}{\left|J_{n}^{(1)}(\varepsilon)\right|} .
$$

As $\varepsilon \downarrow 0$,

$$
R_{N_{t}(\varepsilon)}(\varepsilon) \rightarrow \frac{\sum_{0<s \leq t}\left|\Delta X_{s}\right|-\left|\widetilde{\Delta}_{t}^{(1)}\right|}{\left|\widetilde{\Delta}_{t}^{(1)}\right|}=: R_{t}, \text { a.s. }
$$

and for ${ }^{(1)} \widetilde{X}_{t} / \widetilde{\Delta}_{t}^{(1)} \stackrel{\mathrm{P}}{\longrightarrow} 0$ it suffices that $R_{t} \stackrel{\mathrm{P}}{\longrightarrow} 0$, as $t \downarrow 0$.

We proceed by finding an expression for the Laplace transform of $R_{t}$. We make use of results for "trimmed sums" set out in Goldie and Maller [20] noting that the numerator in $R_{n}(\varepsilon)$ is the "1-trimmed" sum of the i.i.d., nonnegative, rvs $\left|J_{1}(\varepsilon)\right|, \ldots,\left|J_{n}(\varepsilon)\right|$. Let

$$
S_{n}^{(\varepsilon)}(u)=\sum_{i=1}^{n}\left|J_{i}(\varepsilon)(u)\right|, u>0,
$$

where the $J_{i}(\varepsilon)(u)$ are i.i.d. with the distribution of $J_{1}(\varepsilon)$, conditioned on $\left|J_{1}(\varepsilon)\right| \leq$ $u$; similarly, set

$$
S_{n}^{(\varepsilon)}(u-)=\sum_{i=1}^{n}\left|J_{i}(\varepsilon)(u-)\right|, u>0,
$$

where the $J_{i}(\varepsilon)(u-)$ are i.i.d. with the distribution of $J_{1}(\varepsilon)$, conditional on the event $\left|J_{1}(\varepsilon)\right|<u$, and let $h_{\varepsilon}(u):=P\left(\left|J_{1}(\varepsilon)\right| \leq u\right)$. Using similar arguments as in [20], p.245, we can then write, for $x>0$,

$$
n \int_{(\varepsilon, \infty)} P\left(S_{n-1}^{(\varepsilon)}(u) / u \leq x\right) h_{\varepsilon}^{n-1}(u) h_{\varepsilon}(\mathrm{d} u) \leq P\left(R_{n}(\varepsilon) \leq x\right)
$$

and

$$
P\left(R_{n}(\varepsilon) \leq x\right) \leq n \int_{(\varepsilon, \infty)} P\left(S_{n-1}^{(\varepsilon)}(u-) / u \leq x\right) h_{\varepsilon}^{n-1}(u) h_{\varepsilon}(\mathrm{d} u) .
$$

The Laplace transform of $R_{n}(\varepsilon)$ is

$$
\phi_{R_{n}(\varepsilon)}(\lambda)=\int_{[0, \infty)} e^{-\lambda x} P\left(R_{n}(\varepsilon) \in \mathrm{d} x\right)=\lambda \int_{0}^{\infty} e^{-\lambda x} P\left(R_{n}(\varepsilon) \leq x\right) \mathrm{d} x,
$$

hence satisfies, by (5.29) and (5.30),

$$
n \int_{(\varepsilon, \infty)}\left(\phi_{u}^{(\varepsilon)}(\lambda)\right)^{n-1} h_{\varepsilon}^{n-1}(u) \mathrm{d} h_{\varepsilon}(u) \leq \phi_{R_{n}(\varepsilon)}(\lambda)
$$

and

$$
\phi_{R_{n}(\varepsilon)}(\lambda) \leq n \int_{(\varepsilon, \infty)}\left(\phi_{u-}^{(\varepsilon)}(\lambda)\right)^{n-1} h_{\varepsilon}^{n-1}(u) \mathrm{d} h_{\varepsilon}(u)
$$


with $\phi_{u}^{(\varepsilon)}(\lambda)$ and $\phi_{u-}^{(\varepsilon)}(\lambda)$ the Laplace transforms of $\left|J_{1}(\varepsilon)(u)\right| / u$ and $\left|J_{1}(\varepsilon)(u-)\right| / u$. Multiply each side of (5.31) and the following equation by $P\left(N_{t}(\varepsilon)=n\right)$, and sum over $n \geq 0$. Note that

$$
\begin{aligned}
& \sum_{n \geq 0} n \int_{(\varepsilon, \infty)}\left(\phi_{u}^{(\varepsilon)}(\lambda)\right)^{n-1} h_{\varepsilon}^{n-1}(u) h_{\varepsilon}(\mathrm{d} u) P\left(N_{t}(\varepsilon)=n\right) \\
= & t \bar{\Pi}(\varepsilon) e^{-t \bar{\Pi}(\varepsilon)} \int_{(\varepsilon, \infty)} \sum_{n \geq 1}\left(\frac{\int_{\varepsilon<x<u} e^{-\lambda x / u}|\mathrm{~d} \bar{\Pi}(x)|}{\bar{\Pi}(\varepsilon)}\right)^{n-1} \frac{(t \bar{\Pi}(\varepsilon))^{n-1}}{(n-1) !} \frac{|\mathrm{d} \bar{\Pi}(u)|}{\bar{\Pi}(\varepsilon)} \\
= & t \int_{(\varepsilon, \infty)} e^{-t \int_{(\varepsilon, u)}\left(1-e^{-\lambda x / u}\right)|\mathrm{d} \bar{\Pi}(x)|-t \bar{\Pi}(u)|\mathrm{d} \bar{\Pi}(u)|,}
\end{aligned}
$$

with a similar expression for $\phi_{u-}^{(\varepsilon)}(\lambda)$. Define, for $u>0$,

$$
g_{\lambda}(u):=\int_{(0, u)}\left(1-e^{-\lambda x / u}\right)|\mathrm{d} \bar{\Pi}(x)|+\bar{\Pi}(u)
$$

and

$$
\widetilde{g}_{\lambda}(u):=\int_{(0, u]}\left(1-e^{-\lambda x / u}\right)|\mathrm{d} \bar{\Pi}(x)|+\bar{\Pi}(u-) .
$$

Then, letting $\varepsilon \downarrow 0$ in (5.31) and the following equation, and using (5.32), we get the following inequality for the Laplace transform of $R_{t}$ :

$$
t \int_{(0, \infty)} e^{-t \widetilde{g}_{\lambda}(u)}|\mathrm{d} \bar{\Pi}(u)| \leq \phi_{R_{t}}(\lambda) \leq t \int_{(0, \infty)} e^{-t g_{\lambda}(u)}|\mathrm{d} \bar{\Pi}(u)| .
$$

We aim to show that $\bar{\Pi}(x) \in S V$ at 0 implies $\phi_{R_{t}}(\lambda) \rightarrow 1$ for each $\lambda>0$, which will give $R_{t} \stackrel{\mathrm{P}}{\longrightarrow} 0$, as $t \downarrow 0$. For this, first consider the upper bound in (5.33). Integration by parts (Fubini's theorem) and a change of variables shows that

$$
g_{\lambda}(u)=e^{-\lambda} \bar{\Pi}(u)+\lambda \int_{0}^{1} e^{-\lambda x} \bar{\Pi}(x u) \mathrm{d} x .
$$

Thus $g_{\lambda}(u)$ is nonincreasing and right-continuous on $(0, \infty)$, with $g_{\lambda}(0+)=+\infty$

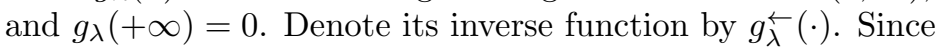

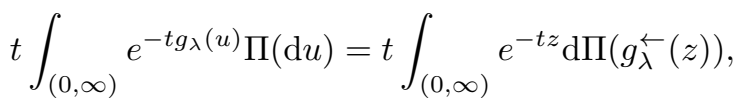

the upper bound in (5.33) tends to 1 as $t \downarrow 0$ if and only if the right-hand side of (5.34) tends to 1 as $t \downarrow 0$, equivalently, by Bingham et al. [5], Theorem 1.7.1, p.37, if $\bar{\Pi}\left(g_{\lambda}^{\leftarrow}(z)\right) \sim z$ as $z \uparrow \infty$, equivalently, again, if $\bar{\Pi}(y) \sim g_{\lambda}(y)$ as $y \downarrow 0$. But, since $\bar{\Pi}(y) \in S V$ at 0 , this is true, because

$$
\frac{g_{\lambda}(y)}{\bar{\Pi}(y)} \rightarrow e^{-\lambda}+\lambda \int_{0}^{1} e^{-\lambda x} \mathrm{~d} x=1
$$

as $y \downarrow 0$. Note that $\bar{\Pi} \in S V$ at 0 implies $\bar{\Pi}(y-) / \bar{\Pi}(y) \rightarrow 1$, as $y \downarrow 0$, because, for all $\eta \in(0,1)$

$$
0 \leq \frac{\bar{\Pi}(y-)-\bar{\Pi}(y)}{\bar{\Pi}(y)} \leq \frac{\bar{\Pi}(y(1-\eta))}{\bar{\Pi}(y)}-1 \rightarrow 0 .
$$

Then similar working shows that the lower bound in (5.33) tends to 1 as $t \downarrow 0$ iff $\bar{\Pi}(y) \sim \widetilde{g}_{\lambda}(y)$ as $y \downarrow 0$, which is the case when $\bar{\Pi}(y) \in S V$ at 0 . Putting the upper 
and lower bounds together, we see that $\phi_{R_{t}}(\lambda) \rightarrow 1$ as $t \downarrow 0$ for each $\lambda>0$, as required.

For the convergence of $V_{t} /\left(\widetilde{\Delta}_{t}^{(1)}\right)^{2}$ to 1 , as $t \downarrow 0$, just note that $V_{t}$ is a Lévy process of bounded variation with zero drift and no normal component, its Lévy measure $\bar{\Pi}_{V}(x)=\bar{\Pi}(\sqrt{x}), x>0$, is also SV at 0 , while $\left(\widetilde{\Delta}_{t}^{(1)}\right)^{2}$ is the jump of $V_{t}$ of largest modulus in $(0, t]$. Now apply the result just obtained for $X$.

Finally, if in addition $X$ is symmetric, then $P\left(\widetilde{\Delta}_{t}^{(1)}<0\right)=P\left(\widetilde{\Delta}_{t}^{(1)}>0\right)=1 / 2$, and the convergence of $Y_{t}$ to $Y$, where $Y= \pm 1$, each with probability $1 / 2$, follows easily.

For the next lemma, define $\Delta \bar{\Pi}(y)=\bar{\Pi}(y-)-\bar{\Pi}(y) \geq 0, y>0$.

Lemma 5.4. For all $x>0$,

$$
\begin{aligned}
& P\left(\widetilde{\Delta}_{t}^{(1)}>x\right) \\
& =\int_{(x, \infty)}\left[t e^{-t \bar{\Pi}(y)} 1_{\{\Delta \bar{\Pi}(y)=0\}}+\left(\frac{e^{-t \bar{\Pi}(y)}-e^{-t \bar{\Pi}(y-)}}{\Delta \bar{\Pi}(y)}\right) 1_{\{\Delta \bar{\Pi}(y) \neq 0\}}\right] \Pi(\mathrm{d} y) .
\end{aligned}
$$

Proof of Lemma 5.4. Now for $x>0$,

$$
P\left(\widetilde{\Delta}_{t}^{(1)}>x\right)=\lim _{\varepsilon \downarrow 0} P\left(J_{N_{t}(\varepsilon)}^{(1)}(\varepsilon)>x\right)=\lim _{\varepsilon \downarrow 0} \sum_{n \geq 0} P\left(J_{n}^{(1)}(\varepsilon)>x\right) P\left(N_{t}(\varepsilon)=n\right) .
$$

A similar calculation as in Kesten and Maller ([28, Eq. 4.22, p.1837) shows that the last term is the limit as $\varepsilon \rightarrow 0$ of

$$
\sum_{n \geq 1} \int_{(x, \infty)} \sum_{j=0}^{n-1} h_{\varepsilon}^{j}(y) h_{\varepsilon}^{n-1-j}(y-) \mathrm{d} F_{\varepsilon}(y) P\left(N_{t}(\varepsilon)=n\right),
$$

where, as previously, $h_{\varepsilon}(x)=P\left(\left|J_{1}(\varepsilon)\right| \leq x\right), x>0$, and we set $F_{\varepsilon}(x)=P\left(J_{1}(\varepsilon) \leq\right.$ $x), x \in \mathbb{R}$. Choose $\varepsilon \in(0, x)$. Straightforward calculations give the last expression equal to

$$
\begin{gathered}
\sum_{n \geq 1}\left(\frac{e^{-t \bar{\Pi}(\varepsilon)}(t \bar{\Pi}(\varepsilon))^{n}}{n !}\right) \int_{(x, \infty)}\left[n h_{\varepsilon}^{n-1}(y) 1_{\left\{\Delta h_{\varepsilon}(y)=0\right\}}\right. \\
\left.+\left(\frac{h_{\varepsilon}^{n}(y)-h_{\varepsilon}^{n}(y-)}{\Delta h_{\varepsilon}(y)}\right) 1_{\left\{\Delta h_{\varepsilon}(y) \neq 0\right\}}\right] \mathrm{d} F_{\varepsilon}(y) \\
=e^{-t \bar{\Pi}(\varepsilon)} \int_{(x, \infty)}\left[t \bar{\Pi}(\varepsilon) e^{t \bar{\Pi}(\varepsilon) h_{\varepsilon}(y)} 1_{\left\{\Delta h_{\varepsilon}(y)=0\right\}}\right. \\
\left.+\left(\frac{e^{t \bar{\Pi}(\varepsilon) h_{\varepsilon}(y)}-e^{t \bar{\Pi}(\varepsilon) h_{\varepsilon}(y-)}}{\Delta h_{\varepsilon}(y)}\right) 1_{\left\{\Delta h_{\varepsilon}(y) \neq 0\right\}}\right] \mathrm{d} F_{\varepsilon}(y) .
\end{gathered}
$$

Substituting

$$
F_{\varepsilon}(y)=1-\frac{\bar{\Pi}^{+}(y)}{\bar{\Pi}(\varepsilon)} 1_{\{y>\varepsilon\}} \text { and } h_{\varepsilon}(y)=1-\frac{\bar{\Pi}(y)}{\bar{\Pi}(\varepsilon)} 1_{\{|y|>\varepsilon\}}
$$

and noting that the resulting expression does not depend on $\varepsilon$, as long as $\varepsilon<x$, gives (5.35). 
Proof of Theorem 3.4. (i) Let the conditions in Part (a) hold, so $\sigma^{2}=0, \bar{\Pi} \in S V$ at 0 , and $\bar{\Pi}^{-}(x)=o\left(\bar{\Pi}^{+}(x)\right)$, as $x \downarrow 0$. Then $X$ is of bounded variation, and we assume its drift is zero. By Lemma 5.3. $X_{t} / \widetilde{\Delta}_{t}^{(1)} \stackrel{\mathrm{P}}{\longrightarrow} 1$ and $V_{t} /\left(\widetilde{\Delta}_{t}^{(1)}\right)^{2} \stackrel{\mathrm{P}}{\longrightarrow} 1$, as $t \downarrow 0$, so it remains to determine the sign of $\widetilde{\Delta}_{t}^{(1)}$.

Taking $x \downarrow 0$, we can write (5.35) in the form

$$
P\left(\widetilde{\Delta}_{t}^{(1)}>0\right)=t \int_{(0, \infty)} e^{-t \bar{\Pi}(y)} \Pi(\mathrm{d} y)-Q_{t},
$$

where

$$
Q_{t}:=\int_{(0, \infty)} e^{-t \bar{\Pi}(y)}\left(\frac{t \Delta \bar{\Pi}(y)+e^{-t \Delta \bar{\Pi}(y)}-1}{\Delta \bar{\Pi}(y)}\right) 1_{\{\Delta \bar{\Pi}(y) \neq 0\}} \Pi(\mathrm{d} y) .
$$

We will show that $Q_{t} \rightarrow 0$ as $t \downarrow 0$, under the assumption $\bar{\Pi} \in S V$ at 0 . As we showed earlier, $\bar{\Pi} \in S V$ at 0 implies $\bar{\Pi}(y-) \sim \bar{\Pi}(y)$ as $y \downarrow 0$; thus $\Delta \bar{\Pi}(y) / \bar{\Pi}(y) \rightarrow 0$, as $y \downarrow 0$. Given $\eta \in(0,1)$, choose $\zeta \in(0,1)$ such that $\Delta \bar{\Pi}(y) \leq \eta \bar{\Pi}(y)$ when $0<y \leq \zeta$. Then use the inequality $0<x+e^{-x}-1 \leq x^{2}$ for $x>0$, together with $\bar{\Pi}(1)=0$, to get

$$
\begin{aligned}
0 \leq Q_{t} & \leq t^{2} \int_{(0,1]} e^{-t \bar{\Pi}(y)} \Delta \bar{\Pi}(y) \Pi(\mathrm{d} y) \\
& \leq \eta t^{2} \int_{(0, \zeta)} e^{-t \bar{\Pi}(y)} \bar{\Pi}(y) \Pi(\mathrm{d} y)+t^{2} \int_{(\zeta, 1]} \Delta \bar{\Pi}(y) \Pi(\mathrm{d} y) .
\end{aligned}
$$

The last term is $o(1)$ as $t \downarrow 0$. In the first term,

$$
\Pi(\mathrm{d} y)=-\mathrm{d} \bar{\Pi}^{+}(y) \leq-\mathrm{d} \bar{\Pi}^{+}(y)-\mathrm{d} \bar{\Pi}^{-}(y)=|\mathrm{d} \bar{\Pi}(y)|,
$$

SO

$$
\begin{aligned}
0 \leq Q_{t} & \leq \eta t^{2} \int_{(0, \zeta)} e^{-t \bar{\Pi}(y)} \bar{\Pi}(y)|\mathrm{d} \bar{\Pi}(y)|+o(1) \\
& \leq \eta t^{2} \int_{0}^{\infty} z e^{-t z} \mathrm{~d} z+o(1)=\eta+o(1), \text { as } t \downarrow 0 .
\end{aligned}
$$

Since $\eta$ is arbitrary, we have $\lim _{t \downarrow 0} Q_{t}=0$ as claimed, so we conclude

$$
P\left(\widetilde{\Delta}_{t}^{(1)}>0\right)=t \int_{(0, \infty)} e^{-t \bar{\Pi}(y)} \Pi(\mathrm{d} y)+o(1), \text { as } t \downarrow 0 .
$$

Using this relation, we see that $P\left(\widetilde{\Delta}_{t}^{(1)}>0\right) \rightarrow 1$ as $t \downarrow 0$ if and only if

$$
t \int_{(0, \infty)} e^{-t z}\left|\mathrm{~d} \bar{\Pi}^{+}\left(\bar{\Pi}^{\leftarrow}(z)\right)\right| \rightarrow 1
$$

as $t \downarrow 0$. Once again using [5], Theorem 1.7.1, p.37, this is the case if and only if $v^{-1} \bar{\Pi}^{+}\left(\bar{\Pi}^{\leftarrow}(v)\right) \rightarrow 1$ as $v \uparrow \infty$, equivalently, if $\bar{\Pi}^{+}(y) \sim \bar{\Pi}(y)$ as $y \downarrow 0$, and this is true if and only if $\bar{\Pi}^{-}(y)=o\left(\bar{\Pi}^{+}(y)\right)$ as $y \downarrow 0$. This is assumed in Part (a), so we have Part (b) via

$$
\frac{X_{t}}{\sqrt{V_{t}}}=\frac{X_{t}}{\widetilde{\Delta}_{t}^{(1)}} \sqrt{\frac{\left(\widetilde{\Delta}_{t}^{(1)}\right)^{2}}{V_{t}}} \operatorname{sgn}\left(\widetilde{\Delta}_{t}^{(1)}\right) \stackrel{\mathrm{P}}{\longrightarrow} 1, \text { as } t \downarrow 0 .
$$

(ii) Conversely, suppose $Y_{t}=X_{t} / \sqrt{V_{t}} \stackrel{\mathrm{P}}{\longrightarrow} 1$ as $t \downarrow 0$. If $\sigma^{2}>0$, then $Y_{t} \stackrel{\mathrm{D}}{\longrightarrow}$ $N(0,1)$ as $t \downarrow 0$, so we must have $\sigma^{2}=0$. 
Symmetrise $X_{t}$ to $X_{t}^{S}:=X_{t}-\widetilde{X}_{t}$, where $\widetilde{X}_{t}$ is an independent copy of $X_{t}$, and let

$$
\begin{aligned}
V_{t}^{S}:=\sum_{0<s \leq t}\left(\Delta X_{s}^{S}\right)^{2} & =\sum_{0<s \leq t}\left(\Delta X_{s}-\Delta \tilde{X}_{s}\right)^{2} \\
& =\sum_{0<s \leq t}\left(\Delta X_{s}\right)^{2}+\sum_{0<s \leq t}\left(\Delta \tilde{X}_{s}\right)^{2}=: V_{t}+\widetilde{V}_{t},
\end{aligned}
$$

where the cross product term vanishes because the independent processes $X$ and $\widetilde{X}$ have no jumps in common, a.s. Write

$$
Y_{t}^{S}:=\frac{X_{t}^{S}}{\sqrt{V_{t}^{S}}}=\frac{X_{t}-\widetilde{X}_{t}}{\sqrt{V_{t}+\widetilde{V}_{t}}}=\frac{X_{t}}{\sqrt{V_{t}}} \sqrt{\frac{V_{t}}{V_{t}+\widetilde{V}_{t}}}-\frac{\widetilde{X}_{t}}{\sqrt{\widetilde{V}_{t}}} \sqrt{\frac{\widetilde{V}_{t}}{V_{t}+\widetilde{V}_{t}}}
$$

which is obviously a symmetric process. Take any sequence $t_{k} \downarrow 0$ and choose a subsequence $t_{k^{\prime}} \downarrow 0$ such that

$$
\frac{V_{t_{k^{\prime}}}}{V_{t_{k^{\prime}}}+\widetilde{V}_{t_{k^{\prime}}}} \stackrel{\mathrm{D}}{\longrightarrow} V^{\prime} \in[0,1]
$$

Since $X_{t} / \sqrt{V_{t}} \stackrel{\mathrm{P}}{\longrightarrow} 1$ and $\widetilde{X}_{t} / \sqrt{\widetilde{V}_{t}} \stackrel{\mathrm{P}}{\longrightarrow} 1$ as $t \downarrow 0$, we have, as $t_{k^{\prime}} \downarrow 0$,

$$
Y_{t_{k^{\prime}}}^{S} \stackrel{\mathrm{D}}{\longrightarrow}\left(Y^{\prime}\right)^{S}:=\sqrt{V^{\prime}}-\sqrt{1-V^{\prime}}=: g\left(V^{\prime}\right),
$$

where $g(v)=\sqrt{v}-\sqrt{1-v}$ is continuous and strictly increasing on $[0,1]$, with $g(0)=-1, g(1)=1$, and $\sup _{0 \leq v \leq 1}|g(v)| \leq 1$. As mentioned following (5.18), $E Y_{t_{k^{\prime}}}^{S}=0, E\left(Y_{t_{k^{\prime}}}^{S}\right)^{2}=1$ and $1 \leq E\left(Y_{t_{k^{\prime}}}^{S}\right)^{4} \leq 3$. So by uniform integrability we have

$$
E\left(g^{2}\left(V^{\prime}\right)\right)=\lim _{t_{k^{\prime}} \downarrow 0} E\left(Y_{t_{k^{\prime}}}^{S}\right)^{2}=1,
$$

which, since $\left|g\left(V^{\prime}\right)\right| \leq 1$, means $\left|g\left(V^{\prime}\right)\right|=1$, a.s. Also, $E\left(g\left(V^{\prime}\right)\right)=0$; hence $\left(Y^{\prime}\right)^{S}=g\left(V^{\prime}\right)= \pm 1$ with probability $1 / 2$ each. This is true for all subsequences, so $Y_{t}^{S} \stackrel{\mathrm{D}}{\longrightarrow} Y^{S}= \pm 1$ with probability $1 / 2$ each, as $t \downarrow 0$. From the case $\beta=0$ in Proposition 5.2 of 34 we now get that $\bar{\Pi}^{S}(x) \in S V$ at 0 , where $\bar{\Pi}^{S}$ is the Lévy measure of $X^{S}$. But $\bar{\Pi}^{S}(x)=2 \bar{\Pi}(x)$, so $\bar{\Pi}(x) \in S V$ at 0 .

Now $\bar{\Pi}(x) \in S V$ at 0 implies $\int_{0}^{1} \bar{\Pi}(x) \mathrm{d} x<\infty$. Therefore by Lemma 4.1 of [1] we can infer that $X_{t}$ is of bounded variation with drift $\delta$, say, and so $X_{t} / t \stackrel{\mathrm{P}}{\longrightarrow} \delta$ as $t \rightarrow 0$. Also, for $\varepsilon>0, t>0$,

$$
\begin{aligned}
P\left(\left|\widetilde{\Delta}_{t}^{(1)}\right|>\varepsilon t\right) & =1-P\left(\text { no jump in } X_{s} \text { until time } t \text { exceeds } \varepsilon t \text { in modulus }\right) \\
& =1-e^{-t \bar{\Pi}(\varepsilon t)} \rightarrow 0, \text { as } t \downarrow 0,
\end{aligned}
$$

since $\bar{\Pi} \in S V$ at 0 . Thus $\widetilde{\Delta}_{t}^{(1)} / t \stackrel{\mathrm{P}}{\longrightarrow} 0$ as $t \downarrow 0$. The slow variation of $\bar{\Pi}$ at 0 together with $\sigma^{2}=0$ also gives by Lemma 5.3 that $V_{t} /\left(\widetilde{\Delta}_{t}^{(1)}\right)^{2} \stackrel{\mathrm{P}}{\longrightarrow} 1$, as $t \downarrow 0$. Then

$$
\frac{X_{t}}{\left|\widetilde{\Delta}_{t}^{(1)}\right|}=\frac{X_{t}}{\sqrt{V_{t}}} \sqrt{\frac{V_{t}}{\left(\widetilde{\Delta}_{t}^{(1)}\right)^{2}}} \stackrel{\mathrm{P}}{\longrightarrow} 1, \text { as } t \downarrow 0,
$$


together with $X_{t} / t \stackrel{\mathrm{P}}{\longrightarrow} \delta$ and $\widetilde{\Delta}_{t}^{(1)} / t \stackrel{\mathrm{P}}{\longrightarrow} 0$ shows that $\delta=0$. But then from

$$
\frac{X_{t}}{\widetilde{\Delta}_{t}^{(1)}}=\frac{X_{t}}{\sqrt{V_{t}}} \sqrt{\frac{V_{t}}{\left(\widetilde{\Delta}_{t}^{(1)}\right)^{2}}} \operatorname{sgn}\left(\widetilde{\Delta}_{t}^{(1)}\right)
$$

we deduce that $\operatorname{sgn}\left(\widetilde{\Delta}_{t}^{(1)}\right) \stackrel{\mathrm{P}}{\longrightarrow} 1$, which we showed above is equivalent to $\bar{\Pi}^{-}(x)=$ $o\left(\bar{\Pi}^{+}(x)\right)$, under the assumption $\bar{\Pi} \in S V$ at 0 .

(iii) Assume (c), so that $X_{t} / \sqrt{V_{t}} \stackrel{\mathrm{P}}{\longrightarrow} c$, as $t \downarrow 0$, where $c \in(0, \infty)$. We can write

$$
\frac{X_{2 t}}{\sqrt{V_{2 t}}}=\frac{X_{t}+\left(X_{2 t}-X_{t}\right)}{\sqrt{V_{t}+\left(V_{2 t}-V_{t}\right)}}=\frac{X_{t}+\widetilde{X}_{t}}{\sqrt{V_{t}+\widetilde{V}_{t}}},
$$

where $\widetilde{X}_{t}$ and $\widetilde{V}_{t}$ are independent copies of $X_{t}$ and $V_{t}$. Thus

$$
\frac{X_{2 t}}{\sqrt{V_{2 t}}}=\frac{X_{t}}{\sqrt{V_{t}}} \sqrt{\frac{V_{t}}{V_{t}+\widetilde{V}_{t}}}+\frac{\widetilde{X}_{t}}{\sqrt{\widetilde{V}_{t}}} \sqrt{\frac{\widetilde{V}_{t}}{V_{t}+\widetilde{V}_{t}}} .
$$

Take any sequence $t_{k} \downarrow 0$ and choose a subsequence $t_{k^{\prime}} \downarrow 0$ such that

$$
\frac{V_{t_{k^{\prime}}}}{V_{t_{k^{\prime}}}+\widetilde{V}_{t_{k^{\prime}}}} \stackrel{\mathrm{D}}{\longrightarrow} V^{\prime} \in[0,1]
$$

Then

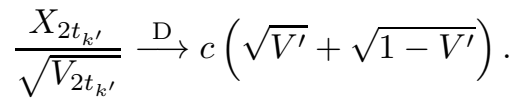

Now also $X_{2 t_{k^{\prime}}} / \sqrt{V_{2 t_{k^{\prime}}}} \stackrel{\mathrm{P}}{\longrightarrow} c$, as $t \downarrow 0$, so, with $h(v):=\sqrt{v}+\sqrt{1-v}$ for $0 \leq v \leq 1$, we have $h\left(V^{\prime}\right)=1$ a.s. But $h(v)>1$ on $(0,1)$, with $h(0)=h(1)=1$, so $h\left(V^{\prime}\right)=1$ a.s. implies $V^{\prime}=0$ a.s., $V^{\prime}=1$ a.s., or $V^{\prime}$ takes values 0 and 1 with probabilities $1 / 2$ each. The first two alternatives imply $V_{t} / \widetilde{V}_{t} \stackrel{\mathrm{P}}{\longrightarrow} 0$ or $V_{t} / \widetilde{V}_{t} \stackrel{\mathrm{P}}{\longrightarrow} \infty$, neither of which is possible because $V_{t} / \widetilde{V}_{t}$ must have mass $1 / 2$ below 1 and above 1 (as $\left.V_{t} \stackrel{\mathrm{D}}{=} \widetilde{V}_{t}\right)$. Thus $V^{\prime}=0$ and 1 with probabilities $1 / 2$ each. Now define

$$
\widehat{Y}_{t}^{S}:=\frac{X_{t}-\widetilde{X}_{t}}{\sqrt{V_{t}+\widetilde{V}_{t}}}=\frac{X_{t}}{\sqrt{V_{t}}} \sqrt{\frac{V_{t}}{V_{t}+\widetilde{V}_{t}}}-\frac{\widetilde{X}_{t}}{\sqrt{\widetilde{V}_{t}}} \sqrt{\frac{\widetilde{V}_{t}}{V_{t}+\widetilde{V}_{t}}} .
$$

The numerator is a symmetrisation of $X_{t}$, and

$$
\widehat{Y}_{t}^{S} \stackrel{\mathrm{D}}{\longrightarrow} c\left(\sqrt{V^{\prime}}-\sqrt{1-V^{\prime}}\right)= \pm c,
$$

with probabilities $1 / 2$ each. Then $E\left(\widehat{Y}_{t}^{S}\right)^{2}=1$ implies $c=1$, so (b) holds.

This completes the proof of Theorem 3.4 .

Remark. We see from (5.36) that $X_{t_{k}} / \sqrt{V_{t_{k}}} \stackrel{\mathrm{P}}{\longrightarrow} 0$, as $t_{k} \downarrow 0$, is not possible for any $t_{k} \downarrow 0$, as we would then have $X_{t_{k}}^{S} / \sqrt{V_{t_{k}}^{S}} \stackrel{\mathrm{P}}{\longrightarrow} 0$, as $t_{k} \downarrow 0$, which is not possible by Theorem 3.2 , 
Proof of Corollary 3.5. Suppose $\left(X_{t}-a(t)\right) / \sqrt{V_{t}} \stackrel{\mathrm{P}}{\longrightarrow} 1$, as $t \downarrow 0$, for a deterministic function $a(t)$. Then we must have $\sigma^{2}=0$. As in the proof of Theorem 3.4, we can take subsequences and find

$$
Y_{t_{k^{\prime}}}^{S} \stackrel{\mathrm{D}}{\longrightarrow} \sqrt{V^{\prime}}-\sqrt{1-V^{\prime}}
$$

and hence $Y_{t}^{S} \stackrel{\mathrm{D}}{\longrightarrow} Y= \pm 1$ with probabilities $1 / 2$ each. Then again from the case $\beta=0$ in Proposition 5.2 of 34 we get that $\bar{\Pi}(x) \in S V$ at 0 . Thus $X_{t}$ is of bounded variation with drift $\delta$, say, and so $X_{t}-t \delta$ is of bounded variation with drift 0 . Lemma 5.3 gives $\left(X_{t}-t \delta\right) / \widetilde{\Delta}_{t}^{(1)} \stackrel{\mathrm{P}}{\longrightarrow} 1$ and $V_{t} /\left(\widetilde{\Delta}_{t}^{(1)}\right)^{2} \stackrel{\mathrm{P}}{\longrightarrow} 1$ as $t \downarrow 0$. Thus

$$
\frac{X_{t}-t \delta}{\sqrt{V_{t}}}=\left(1+o_{P}(1)\right) \operatorname{sgn}\left(\widetilde{\Delta}_{t}^{(1)}\right)
$$

as $t \downarrow 0$. But then

$$
\begin{aligned}
\frac{a(t)-t \delta}{\sqrt{V_{t}}} & =\frac{\left(a(t)-X_{t}\right)+\left(X_{t}-t \delta\right)}{\sqrt{V_{t}}} \\
& =-1+\left(1+o_{P}(1)\right) \operatorname{sgn}\left(\widetilde{\Delta}_{t}^{(1)}\right)=\operatorname{sgn}\left(\widetilde{\Delta}_{t}^{(1)}\right)-1+o_{P}(1) .
\end{aligned}
$$

Now the left-hand side cannot oscillate in sign, $\operatorname{so~} \operatorname{sgn}\left(\widetilde{\Delta}_{t}^{(1)}\right) \stackrel{\mathrm{P}}{\longrightarrow} 1$ and therefore $(a(t)-t \delta) / \sqrt{V_{t}} \stackrel{\mathrm{P}}{\longrightarrow} 0$ as $t \downarrow 0$. We then get $\bar{\Pi}^{-}(x)=o\left(\bar{\Pi}^{+}(x)\right)$, as $x \downarrow 0$.

Conversely, these imply $\left(X_{t}-a(t)\right) / \sqrt{V_{t}} \stackrel{\mathrm{P}}{\longrightarrow}+1$, as $t \downarrow 0$.

Preparatory to proving Theorem 3.6. we generalise Prop. 1 of Mason [35].

Proposition 5.5. Suppose $T_{t}:=X_{t} / \sqrt{V_{t}}$ is relatively compact as $t \downarrow 0$, and also that $\lim \sup _{x \downarrow 0} x^{2} \bar{\Pi}(x) / V(x)=\infty$. Then there is a sequence $t_{k} \downarrow 0$ such that

$$
\lim _{\delta \downarrow 0} \limsup _{t_{k} \downarrow 0} P\left(|| T_{t_{k}}|-1| \leq \delta\right)>0 .
$$

Proof of Proposition 5.5. Recall that we assume $\bar{\Pi}(1)=0$ and $\bar{\Pi}(0+)=\infty$. As in (4.18), let $R(x):=x^{2} \bar{\Pi}(x) / V(x)$. Assume that $T_{t}$ is relatively compact as $t \downarrow 0$ and that $\lim \sup _{x \downarrow 0} R(x)=\infty$. Since we always have $x^{2} \bar{\Pi}(x) \rightarrow 0$ as $x \downarrow 0$, the latter implies that $\sigma^{2}=0$. Choose $\zeta_{k} \downarrow 0$ such that (4.19) holds. Recall that $U(x) \geq x^{2} \bar{\Pi}(x)$ for all $x>0$, so for $\lambda>0$,

$$
0 \leq 1-\frac{\left(\lambda \zeta_{k}\right)^{2} \bar{\Pi}\left(\lambda \zeta_{k}\right)}{U\left(\lambda \zeta_{k}\right)}=\frac{V\left(\lambda \zeta_{k}\right)}{U\left(\lambda \zeta_{k}\right)} \leq \frac{V\left(\lambda \zeta_{k}\right)}{\left(\lambda \zeta_{k}\right)^{2} \bar{\Pi}\left(\lambda \zeta_{k}\right)}=\frac{1}{R\left(\lambda \zeta_{k}\right)} \rightarrow 0,
$$

uniformly in $\lambda \in\left[\lambda_{1}, \lambda_{2}\right]$, where $0<\lambda_{1}<1<\lambda_{2}<\infty$. Now

$$
\int_{\lambda_{1}}^{\lambda_{2}} \frac{\left(s \zeta_{k}\right)^{2} \bar{\Pi}\left(s \zeta_{k}\right)}{U\left(s \zeta_{k}\right)} \frac{\mathrm{d} s}{s}=\frac{1}{2} \int_{\lambda_{1} \zeta_{k}}^{\lambda_{2} \zeta_{k}} \frac{\mathrm{d} U(s)}{U(s)}=\frac{1}{2} \log \left(\frac{U\left(\lambda_{2} \zeta_{k}\right)}{U\left(\lambda_{1} \zeta_{k}\right)}\right)
$$

(recall that $U(x)$ is continuous at each $x>0$ ). The left-hand side of the last expression tends to $\int_{\lambda_{1}}^{\lambda_{2}} \mathrm{~d} s / s=\log \left(\lambda_{2} / \lambda_{1}\right)$, so we have $U\left(\lambda_{2} \zeta_{k}\right) / U\left(\lambda_{1} \zeta_{k}\right) \rightarrow\left(\lambda_{2} / \lambda_{1}\right)^{2}$. Then by (5.38), $\bar{\Pi}\left(\lambda_{2} \zeta_{k}\right) / \bar{\Pi}\left(\lambda_{1} \zeta_{k}\right) \rightarrow 1$, and so we deduce

$$
\lim _{k \rightarrow \infty} \sup _{0<\lambda_{1} \leq \lambda \leq \lambda_{2}}\left|\frac{\bar{\Pi}\left(\lambda \zeta_{k}\right)}{\bar{\Pi}\left(\zeta_{k}\right)}-1\right|=0, \text { for each } 0<\lambda_{1}<\lambda_{2} .
$$


Recall the definition of $X_{t}(\varepsilon)$ in (5.1) (in which we take $\sigma^{2}=0$ ), and of $J_{N_{t}(\varepsilon)}^{(1)}(\varepsilon)$ in (5.25), and similarly let $J_{N_{t}(\varepsilon)}^{(2)}(\varepsilon)$ denote the term among $J_{1}(\varepsilon), \ldots, J_{N_{t}(\varepsilon)}$ of second largest modulus. Define

$$
{ }^{(1)} \widetilde{X}_{t}(\varepsilon):=X_{t}(\varepsilon)-J_{N_{t}(\varepsilon)}^{(1)}(\varepsilon)=t \nu(\varepsilon)+\sum_{i=1}^{N_{t}(\varepsilon)} J_{i}(\varepsilon)-J_{N_{t}(\varepsilon)}^{(1)}(\varepsilon)
$$

and

$$
{ }^{(1)} V_{t}(\varepsilon):=V_{t}(\varepsilon)-\left|J_{N_{t}(\varepsilon)}^{(1)}(\varepsilon)\right|^{2} .
$$

Put $t_{k}:=1 / \bar{\Pi}\left(\zeta_{k}\right)$, and recall that $\bar{\Pi}(0+)=\infty$, so $t_{k} \downarrow 0$. For $\varepsilon>0, \delta>0$, and $0<\lambda_{1}<\lambda_{2}$, define the events

$$
\begin{gathered}
A_{k}(\varepsilon):=\left\{\left|J_{N_{t_{k}}(\varepsilon)}^{(2)}(\varepsilon)\right| \leq \lambda_{1} \zeta_{k}<\lambda_{2} \zeta_{k}<\left|J_{N_{t_{k}}(\varepsilon)}^{(1)}(\varepsilon)\right|\right\}, \\
B_{k}(\varepsilon, \delta):=\left\{\left|{ }^{(1)} \widetilde{X}_{t_{k}}(\varepsilon)\right|>\delta\left|J_{N_{t_{k}}(\varepsilon)}^{(1)}(\varepsilon)\right|\right\}
\end{gathered}
$$

and

$$
C_{k}(\varepsilon, \delta):=\left\{{ }^{(1)} V_{t_{k}}(\varepsilon)>\delta^{2}\left|J_{N_{t_{k}}(\varepsilon)}^{(1)}(\varepsilon)\right|^{2}\right\} .
$$

In the following, we will keep $\varepsilon<\lambda_{1} \zeta_{k}$ (and, later, let $\varepsilon \downarrow 0$ before $k \rightarrow \infty$ ). A straightforward calculation gives

$$
P\left(A_{k}(\varepsilon)\right)=t_{k} \bar{\Pi}\left(\lambda_{2} \zeta_{k}\right) e^{-t_{k} \bar{\Pi}\left(\lambda_{1} \zeta_{k}\right)}=: \rho_{k}\left(\lambda_{1}, \lambda_{2}\right), \text { say. }
$$

Also, on $A_{k}(\varepsilon)$, we have

$$
\begin{aligned}
B_{k}(\varepsilon, \delta) & \subseteq\left\{\left|t_{k} \nu(\varepsilon)+\sum_{i=1}^{N_{t_{k}}(\varepsilon)} J_{i}(\varepsilon) \mathbf{1}_{\left\{\left|J_{i}(\varepsilon)\right| \leq \lambda_{1} \zeta_{k}\right\}}\right|>\delta \lambda_{2} \zeta_{k}\right\} \\
& =\left\{\left|t_{k} \nu(\varepsilon)+\sum_{i=1}^{N_{t_{k}}(\varepsilon)} J_{i}^{k}(\varepsilon)\right|>\delta \lambda_{2} \zeta_{k}\right\},
\end{aligned}
$$

where $J_{i}^{k}(\varepsilon):=J_{i}(\varepsilon) \mathbf{1}_{\left\{\left|J_{i}(\varepsilon)\right| \leq \lambda_{1} \zeta_{k}\right\}}$. Note that

$$
E\left(J_{1}^{k}(\varepsilon)\right)=\int_{\varepsilon<|x| \leq \lambda_{1} \zeta_{k}} x \Pi(\mathrm{d} x) / \bar{\Pi}(\varepsilon)
$$

and $E\left(N_{t_{k}}(\varepsilon)\right)=t_{k} \bar{\Pi}(\varepsilon)$, so we can write

$$
\begin{aligned}
t_{k} \nu(\varepsilon) & +\sum_{i=1}^{N_{t_{k}}(\varepsilon)} J_{i}^{k}(\varepsilon)=t_{k} \nu(\varepsilon)+\sum_{i=1}^{N_{t_{k}}(\varepsilon)}\left(J_{i}^{k}(\varepsilon)-E\left(J_{1}^{k}(\varepsilon)\right)\right) \\
& +\left(N_{t_{k}}(\varepsilon)-E\left(N_{t_{k}}(\varepsilon)\right)\right) E\left(J_{1}^{k}(\varepsilon)\right)+t_{k} \int_{\varepsilon<|x| \leq \lambda_{1} \zeta_{k}} x \Pi(\mathrm{d} x) .
\end{aligned}
$$

Now since $T_{t}$ is assumed relatively compact, we have $x|\nu(x)| \leq M\left(x^{2} \bar{\Pi}(x)+V(x)\right)$, for $x \leq$ some $x_{0}$, for some $M \in(0, \infty)$, by (3.1). Further, by (5.38), $V\left(\lambda \zeta_{k}\right) \leq$ 
$\left(\lambda \zeta_{k}\right)^{2} \bar{\Pi}\left(\lambda \zeta_{k}\right)$, for $k$ large, uniformly in $\lambda \in\left[\lambda_{1}, \lambda_{2}\right]$. Thus for $k$ large, firstly,

$$
\begin{aligned}
\left|t_{k} \nu(\varepsilon)+t_{k} \int_{\varepsilon<|x| \leq \lambda_{1} \zeta_{k}} x \Pi(\mathrm{d} x)\right| & =t_{k}\left|\gamma-\int_{\varepsilon<|x| \leq 1} x \Pi(\mathrm{d} x)+\int_{\varepsilon<|x| \leq \lambda_{1} \zeta_{k}} x \Pi(\mathrm{d} x)\right| \\
& =t_{k}\left|\nu\left(\lambda_{1} \zeta_{k}\right)\right| \\
& \leq 2 M t_{k}\left(\lambda_{1} \zeta_{k}\right) \overline{\Pi\left(\lambda_{1} \zeta_{k}\right)} \\
& \left.\leq 4 M \lambda_{1} \zeta_{k} \quad \text { by (15.40)}, \text { and } t_{k}=1 / \bar{\Pi}\left(\zeta_{k}\right)\right) \\
& \leq(\delta / 2) \lambda_{2} \zeta_{k},
\end{aligned}
$$

for $\lambda_{2}>\lambda_{1}$ large enough. Secondly,

$$
\begin{aligned}
\operatorname{Var}\left(\sum_{i=1}^{N_{t_{k}}(\varepsilon)}\left(J_{i}^{k}(\varepsilon)-E\left(J_{1}^{k}(\varepsilon)\right)\right)\right) & =E\left(N_{t_{k}}(\varepsilon)\right) \operatorname{Var}\left(J_{1}^{k}(\varepsilon)\right) \\
& \leq t_{k} \bar{\Pi}(\varepsilon) \int_{\varepsilon<|x| \leq \lambda_{1} \zeta_{k}} x^{2} \Pi(\mathrm{d} x) / \bar{\Pi}(\varepsilon) \\
& \leq t_{k} V\left(\lambda_{1} \zeta_{k}\right) .
\end{aligned}
$$

Third, using Cauchy-Schwarz,

$$
\begin{aligned}
\operatorname{Var}\left[\left(N_{t_{k}}(\varepsilon)-E\left(N_{t_{k}}(\varepsilon)\right)\right) E\left(J_{1}^{k}(\varepsilon)\right)\right] & =t_{k} \bar{\Pi}(\varepsilon)\left(\int_{\varepsilon<|x| \leq \lambda_{1} \zeta_{k}} x \Pi(\mathrm{d} x) / \bar{\Pi}(\varepsilon)\right)^{2} \\
& \leq t_{k} \int_{\varepsilon<|x| \leq \lambda_{1} \zeta_{k}} x^{2} \Pi(\mathrm{d} x) \\
& \leq t_{k} V\left(\lambda_{1} \zeta_{k}\right) .
\end{aligned}
$$

Putting the three estimates into (5.42) and using Chebychev's inequality, we find that for $\delta_{1}>0$,

$$
\begin{aligned}
& P\left(B_{k}\left(\varepsilon, \delta_{1}\right) \cap A_{k}(\varepsilon)\right) \\
& \leq P\left(\left|\sum_{i=1}^{N_{t_{k}}(\varepsilon)}\left(J_{i}^{k}(\varepsilon)-E\left(J_{1}^{k}(\varepsilon)\right)\right)+\left(N_{t_{k}}(\varepsilon)-E\left(N_{t_{k}}(\varepsilon)\right)\right) E\left(J_{1}^{k}(\varepsilon)\right)\right|>\frac{\delta_{1} \lambda_{2} \zeta_{k}}{2}\right) \\
& \leq \frac{32 t_{k} V\left(\lambda_{1} \zeta_{k}\right)}{\left(\delta_{1} \lambda_{2} \zeta_{k}\right)^{2}} .
\end{aligned}
$$

By a similar argument as in (5.42) and Markov's inequality we get for $\delta_{2}>0$,

$$
\begin{aligned}
P\left(C_{k}\left(\varepsilon, \delta_{2}\right) \cap A_{k}(\varepsilon)\right) & \leq P\left(\sum_{i=1}^{N_{t_{k}}(\varepsilon)}\left(J_{i}^{k}(\varepsilon)\right)^{2}>\left(\delta_{2} \lambda_{2} \zeta_{k}\right)^{2}\right) \\
& \leq \frac{E\left(N_{t_{k}}(\varepsilon)\right) E\left(\left(J_{1}^{k}(\varepsilon)\right)^{2}\right)}{\left(\delta_{2} \lambda_{2} \zeta_{k}\right)^{2}} \leq \frac{t_{k} V\left(\lambda_{1} \zeta_{k}\right)}{\left(\delta_{2} \lambda_{2} \zeta_{k}\right)^{2}}
\end{aligned}
$$

Putting these together gives

$$
\begin{aligned}
P\left(\left\{B_{k}\left(\varepsilon, \delta_{2}\right) \cap A_{k}(\varepsilon)\right\} \cup\left\{C_{k}\left(\varepsilon, \delta_{1}\right) \cap A_{k}(\varepsilon)\right\}\right) & \leq\left(\frac{1}{\delta_{1}^{2}}+\frac{1}{\delta_{2}^{2}}\right) \frac{32 t_{k} V\left(\lambda_{1} \zeta_{k}\right)}{\left(\lambda_{2} \zeta_{k}\right)^{2}} \\
& =: \eta_{k}\left(\delta_{1}, \delta_{2}\right), \text { say. }
\end{aligned}
$$


Now, since $V_{t}(\varepsilon)={ }^{(1)} V_{t}(\varepsilon)+\left|J_{N_{t}(\varepsilon)}^{(1)}(\varepsilon)\right|^{2} \geq\left|J_{N_{t}(\varepsilon)}^{(1)}(\varepsilon)\right|^{2}$, we can write, for $\delta>0$,

$$
\begin{aligned}
& P\left(\left|\frac{\left|X_{t_{k}}(\varepsilon)\right|}{\sqrt{V_{t_{k}}(\varepsilon)}}-1\right|>\delta\right) \\
\leq & P\left(|| X_{t_{k}}(\varepsilon)\left|-\sqrt{V_{t_{k}}(\varepsilon)}\right|>\delta \sqrt{V_{t_{k}}(\varepsilon)}\right) \\
\leq & P\left(\left\{|| X_{t_{k}}(\varepsilon)\left|-\sqrt{V_{t_{k}}(\varepsilon)}\right|>\delta\left|J_{N_{t_{k}}(\varepsilon)}^{(1)}(\varepsilon)\right|,{ }^{(1)} V_{t_{k}}(\varepsilon) \leq(\delta / 2)^{2}\left|J_{N_{t_{k}}(\varepsilon)}^{(1)}(\varepsilon)\right|^{2}\right\}\right. \\
& \left.\cup\left\{{ }^{(1)} V_{t_{k}}(\varepsilon)>(\delta / 2)^{2}\left|J_{N_{t_{k}}(\varepsilon)}^{(1)}(\varepsilon)\right|^{2}\right\}\right) .
\end{aligned}
$$

The latter does not exceed

$$
P\left(\left\{\left|X_{t_{k}}(\varepsilon)-J_{N_{t_{k}}(\varepsilon)}^{(1)}(\varepsilon)\right|>\delta\left|J_{N_{t_{k}}(\varepsilon)}^{(1)}(\varepsilon)\right| / 2\right\} \cup\left\{{ }^{(1)} V_{t_{k}}(\varepsilon)>(\delta / 2)^{2}\left|J_{N_{t}(\varepsilon)}^{(1)}(\varepsilon)\right|^{2}\right\}\right),
$$

because $\sqrt{{ }^{(1)} V_{t_{k}}(\varepsilon)} \leq(\delta / 2)\left|J_{N_{t_{k}}(\varepsilon)}^{(1)}(\varepsilon)\right|$, which implies

$$
\left|\sqrt{V_{t_{k}}(\varepsilon)}-\right| J_{N_{t_{k}}(\varepsilon)}^{(1)}(\varepsilon)|| \leq(\delta / 2)\left|J_{N_{t_{k}}(\varepsilon)}^{(1)}(\varepsilon)\right|,
$$

together with

$$
|| X_{t_{k}}(\varepsilon)\left|-\sqrt{V_{t_{k}}(\varepsilon)}\right|>\delta\left|J_{N_{t_{k}}(\varepsilon)}^{(1)}(\varepsilon)\right|>\left|\sqrt{V_{t_{k}}(\varepsilon)}-\right| J_{N_{t_{k}}(\varepsilon)}^{(1)}(\varepsilon)||,
$$

imply

$$
\begin{aligned}
& \left|X_{t_{k}}(\varepsilon)-J_{N_{t_{k}}(\varepsilon)}^{(1)}(\varepsilon)\right| \\
\geq & || X_{t_{k}}(\varepsilon)|-| J_{N_{t_{k}}(\varepsilon)}^{(1)}(\varepsilon)|| \geq||\left|X_{t_{k}}(\varepsilon)\right|-\sqrt{V_{t_{k}}(\varepsilon)}|-| \sqrt{V_{t_{k}}(\varepsilon)}-\left|J_{N_{t_{k}}(\varepsilon)}^{(1)}(\varepsilon)\right||| \\
= & || X_{t_{k}}(\varepsilon)\left|-\sqrt{V_{t_{k}}(\varepsilon)}\right|-\left|\sqrt{V_{t_{k}}(\varepsilon)}-\right| J_{N_{t_{k}}(\varepsilon)}^{(1)}(\varepsilon)|| \\
\geq & (\delta-\delta / 2)\left|J_{N_{t_{k}}(\varepsilon)}^{(1)}(\varepsilon)\right|=\delta\left|J_{N_{t}(\varepsilon)}^{(1)}(\varepsilon)\right| / 2 .
\end{aligned}
$$

Observe that (5.44) does not exceed $P\left(B_{k}(\varepsilon, \delta / 2) \cup C_{k}(\varepsilon, \delta / 2)\right)$. Argue that, by (5.43) and (5.41),

$$
\begin{aligned}
P\left(B_{k}\left(\varepsilon, \delta_{1}\right) \cup C_{k}\left(\varepsilon, \delta_{2}\right)\right) \leq & P\left(\left\{B_{k}\left(\varepsilon, \delta_{1}\right) \cap A_{k}(\varepsilon)\right\} \cup\left\{C_{k}\left(\varepsilon, \delta_{2}\right) \cap A_{k}(\varepsilon)\right\}\right) \\
& +1-P\left(A_{k}(\varepsilon)\right) \\
\leq & \eta_{k}\left(\delta_{1}, \delta_{2}\right)+1-\rho_{k}\left(\lambda_{1}, \lambda_{2}\right),
\end{aligned}
$$

provided $0<\varepsilon<\lambda_{1} \zeta_{k}$, as we have ensured. Thus by (5.44),

$$
P\left(\left|\frac{\left|X_{t_{k}}(\varepsilon)\right|}{\sqrt{V_{t_{k}}(\varepsilon)}}-1\right|>\delta\right) \leq \eta_{k}(\delta / 2, \delta / 2)+1-\rho_{k}\left(\lambda_{1}, \lambda_{2}\right) .
$$

Now by (5.38) and (5.40),

$$
t_{k} V\left(\lambda_{1} \zeta_{k}\right)=o\left(t_{k} \zeta_{k}^{2} \bar{\Pi}\left(\lambda_{1} \zeta_{k}\right)\right)=o\left(\zeta_{k}^{2}\right)
$$

so $\eta_{k}\left(\delta_{1}, \delta_{2}\right) \rightarrow 0$ as $k \rightarrow \infty$, while, by (5.40), $\rho_{k}\left(\lambda_{1}, \lambda_{2}\right) \rightarrow e^{-1}$ as $k \rightarrow \infty$. Letting $\varepsilon \downarrow 0$ then $k \rightarrow \infty$ in (5.45) gives

$$
\limsup _{t_{k} \downarrow 0} P\left(\left|\frac{\left|X_{t_{k}}\right|}{\sqrt{V_{t_{k}}}}-1\right|>\delta\right) \leq 1-e^{-1}<1,
$$

so (5.37) holds. 
Proof of Theorem 3.6. We have $\bar{\Pi}(1)=0, \bar{\Pi}(0+)=\infty$, and we can assume that $\sigma^{2}=0$.

Now assume $X_{t} / \sqrt{V_{t}} \stackrel{\mathrm{D}}{\longrightarrow} N(0,1)$. We will prove (2.16). $X_{t} / \sqrt{V_{t}} \stackrel{\mathrm{D}}{\longrightarrow} N(0,1)$ implies $X_{t} / \sqrt{V_{t}}=O_{P}(1)$, as $t \downarrow 0$, so by Theorem 3.1

$$
\limsup _{x \downarrow 0} \frac{x|\nu(x)|}{x^{2} \bar{\Pi}(x)+V(x)}<\infty .
$$

Further, by Proposition 5.5 we must have

$$
\limsup _{x \downarrow 0} \frac{x^{2} \bar{\Pi}(x)}{V(x)}<\infty
$$

(because $X_{t} / \sqrt{V_{t}} \stackrel{\mathrm{D}}{\longrightarrow} N(0,1)$ is clearly incompatible with $(5.37)$ ). We deduce from these that (2.11) holds. Hence by Part (ii) of Theorem 2.3 there is a function $b(t)>0$ such that every sequence $t_{k} \downarrow 0$ contains a subsequence $t_{k^{\prime}} \downarrow 0$ for which

$$
\left(\frac{X_{t_{k^{\prime}}}}{b\left(t_{k^{\prime}}\right)}, \frac{V_{t_{k^{\prime}}}}{b^{2}\left(t_{k^{\prime}}\right)}\right) \stackrel{\mathrm{D}}{\longrightarrow}\left(\beta^{\prime}+\tau^{\prime} Z+\mathcal{I}^{\prime},\left(\tau^{\prime}\right)^{2}+\mathcal{J}^{\prime}\right),
$$

for some constants $\beta^{\prime} \in \mathbb{R}$ and $\left(\tau^{\prime}\right)^{2} \geq 0$, with $Z$ standard normal, independent of $\left(\mathcal{I}^{\prime}, \mathcal{J}^{\prime}\right)$, and $P\left(\left(\tau^{\prime}\right)^{2}+\mathcal{J}^{\prime} \leq 0\right)=0$. By Eqs (4.8) and (4.9) of [34, the right-hand side has characteristic function

$$
\begin{aligned}
& \exp \left(-\frac{1}{2} \theta_{1}^{2}\left(\tau^{\prime}\right)^{2}+\int_{\mathbb{R} \backslash\{0\}}\left(e^{\mathrm{i}\left(\theta_{1} x+\theta_{2} x^{2}\right)}-1-\mathrm{i}\left(\theta_{1} x+\theta_{2} x^{2}\right) \mathbf{1}_{\{|x| \leq 1\}}\right) \Lambda^{\prime}(\mathrm{d} x)\right) \\
& \quad \times \exp \left(\mathrm{i} \theta_{1} \beta^{\prime}+\mathrm{i} \theta_{2}\left(\left(\tau^{\prime}\right)^{2}+\int_{|x| \leq 1} x^{2} \Lambda^{\prime}(\mathrm{d} x)\right)\right) .
\end{aligned}
$$

Now we need some calculations. For these, we temporarily drop the prime on $\Lambda^{\prime}$. Let

$$
\phi\left(\theta_{1}, \theta_{2}\right)=\exp \left(\int_{\mathbb{R} \backslash\{0\}}\left(e^{\mathrm{i}\left(\theta_{1} x+\theta_{2} x^{2}\right)}-1-\mathrm{i}\left(\theta_{1} x+\theta_{2} x^{2}\right) \mathbf{1}_{\{|x| \leq 1\}}\right) \Lambda(\mathrm{d} x)\right) .
$$

We can write

$$
\phi\left(\theta_{1}, \theta_{2}\right)=\phi_{+}\left(\theta_{1}, \theta_{2}\right) \phi_{-}\left(\theta_{1}, \theta_{2}\right)
$$

where

$$
\begin{aligned}
& \phi_{ \pm}\left(\theta_{1}, \theta_{2}\right) \\
= & \exp \left(\int_{0}^{\infty}\left(e^{\mathrm{i}\left( \pm \theta_{1} x+\theta_{2} x^{2}\right)}-1-\mathrm{i}\left( \pm \theta_{1} x+\theta_{2} x^{2}\right) \mathbf{1}_{\{x \leq 1\}}\right)\left|\mathrm{d} \bar{\Lambda}^{ \pm}(x)\right|\right)
\end{aligned}
$$

(and, throughout, the upper/lower signs are to be taken together). For $s>0$, set

$$
\varphi_{ \pm}(s)=\sup \left\{y: \bar{\Lambda}^{ \pm}(y)>s\right\} .
$$

Notice that each $\varphi_{ \pm}$is left continuous and nonincreasing on $(0, \infty)$, and since $\bar{\Lambda}^{+}$ and $\bar{\Lambda}^{-}$are right continuous,

$$
\bar{\Lambda}^{ \pm}(y)=\inf \left\{s: \varphi_{ \pm}(s) \leq y\right\}, y>0 .
$$


By the change of variables $x=\varphi_{ \pm}(s)$ we get

$$
\begin{aligned}
& \phi_{ \pm}\left(\theta_{1}, \theta_{2}\right) \\
= & \exp \left(\int_{0}^{\infty}\left(e^{\mathrm{i}\left( \pm \theta_{1} \varphi_{ \pm}(s)+\theta_{2} \varphi_{ \pm}^{2}(s)\right)}-1-\mathrm{i}\left( \pm \theta_{1} \varphi_{ \pm}(s)+\theta_{2} \varphi_{ \pm}^{2}(s)\right) \mathbf{1}_{\left\{s \geq \bar{\Lambda}^{ \pm}(1)\right\}}\right) \mathrm{d} s\right) .
\end{aligned}
$$

Moreover, since

$$
\int_{0}^{\infty} \varphi_{+}^{2}(s) \mathbf{1}_{\left\{s \geq \bar{\Lambda}^{+}(1)\right\}} \mathrm{d} s+\int_{0}^{\infty} \varphi_{-}^{2}(s) \mathbf{1}_{\left\{s \geq \bar{\Lambda}^{-}(1)\right\}} \mathrm{d} s=\int_{\mathbb{R} \backslash\{0\}} x^{2} \mathbf{1}_{\{|x| \leq 1\}} \Lambda(\mathrm{d} x)<\infty,
$$

we see that for any $\delta>0$,

$$
\int_{\delta}^{\infty} \varphi_{ \pm}^{2}(s) d s<\infty
$$

( $\delta$ can be taken as 0 if one or both of $\bar{\Lambda}^{ \pm}(1)=0$, but we do not assume this.) Recall the notation in (5.18), where

$$
S_{i}=\sum_{j=1}^{i} \varpi_{j}, i=1,2, \ldots,
$$

and $\varpi_{1}, \varpi_{2}, \ldots$ are i.i.d. exponential random variables with mean 1 . Define $M(t)=$ $\sum_{j=1}^{\infty} 1\left\{S_{j} \leq t\right\}, t \geq 0$, a Poisson process on $[0, \infty)$ with rate 1 . For any $c>0$, introduce the random variable

$$
V(c)=\int_{0}^{c} \Psi(s) \mathrm{d} M(s)+\int_{c}^{\infty} \Psi(s) \mathrm{d}\{M(s)-s\},
$$

where $\Psi$ is a left continuous function defined on $(0, \infty)$.

Lemma 5.6. Assume $\int_{\delta}^{\infty} \Psi^{2}(s) d s<\infty$ for all $\delta>0$. Then for any $c>0$,

$$
E e^{\mathrm{i} V(c)}=\exp \left(\int_{0}^{\infty}\left(e^{\mathrm{i} \Psi(s)}-1-i \Psi(s) \mathbf{1}_{\{s \geq c\}}\right) \mathrm{d} s\right) .
$$

Proof of Lemma 5.6. Choose any $T>c$. We can write

$$
\begin{gathered}
V(c)=\int_{0}^{T} \Psi(s) \mathrm{d} M(s)-\int_{c}^{T} \Psi(s) \mathrm{d} s+\int_{T}^{\infty} \Psi(s) \mathrm{d}\{M(s)-s\} \\
=: V_{T}(c)+\int_{T}^{\infty} \Psi(s) \mathrm{d}\{M(s)-s\} .
\end{gathered}
$$

Since

$$
E\left(\int_{T}^{\infty} \Psi(s) \mathrm{d}\{M(s)-s\}\right)^{2}=\int_{T}^{\infty} \Psi^{2}(s) \mathrm{d} s \rightarrow 0, \text { as } T \rightarrow \infty,
$$

we get that

$$
\int_{T}^{\infty} \Psi(s) \mathrm{d}\{M(s)-s\} \stackrel{\mathrm{P}}{\longrightarrow} 0, \text { as } T \rightarrow \infty .
$$

Therefore to finish the proof it suffices to show that $E \exp \left(i V_{T}(c)\right)$ tends to the right-hand side of (5.49), as $T \rightarrow \infty$. Now

$$
V_{T}(c)=\sum_{i=1}^{M(T)} \Psi\left(S_{i}\right)-\int_{c}^{T} \Psi(s) d s,
$$


where the sum is defined to be zero if $M(T)=0$. Since $\left(S_{1}, \ldots, S_{k}\right)$ given $M(T)=k$ has the same distribution as the order statistics based on $k$ independent uniform $(0, T)$ random variables, the same calculation as in Lemma 5.1 of 34 gives

$$
E e^{\mathrm{i} V_{T}(c)}=\exp \left(\int_{0}^{T}\left(e^{i \Psi(s)}-1-i \Psi(s) \mathbf{1}_{\{s \geq c\}}\right) \mathrm{d} s\right) .
$$

Letting $T \rightarrow \infty$ we conclude (5.49).

Next let $\Psi_{ \pm}\left(s, \theta_{1}, \theta_{2}\right)=\theta_{1} \varphi_{ \pm}(s)+\theta_{2} \varphi_{ \pm}^{2}(s)$, and

$$
V_{ \pm}\left(\theta_{1}, \theta_{2}\right)=\int_{0}^{\bar{\Lambda}^{ \pm}(1)} \Psi_{ \pm}\left(s, \theta_{1}, \theta_{2}\right) \mathrm{d} M_{ \pm}(s)+\int_{\bar{\Lambda}^{ \pm}(1)}^{\infty} \Psi_{ \pm}\left(s, \theta_{1}, \theta_{2}\right) \mathrm{d}\left\{M_{ \pm}(s)-s\right\},
$$

where $M_{+}$and $M_{-}$are independent Poisson processes on $[0, \infty)$ with rate 1 . Applying (5.48) and Lemma 5.6 we get that

$$
\phi\left(\theta_{1}, \theta_{2}\right)=\phi_{+}\left(\theta_{1}, \theta_{2}\right) \phi_{-}\left(\theta_{1}, \theta_{2}\right)=E e^{\mathrm{i} V_{+}\left(\theta_{1}, \theta_{2}\right)} E e^{\mathrm{i} V_{-}\left(\theta_{1}, \theta_{2}\right)} .
$$

Define

$$
\begin{aligned}
W:=\int_{0}^{\bar{\Lambda}^{+}(1)} \varphi_{+}(s) \mathrm{d} M_{+}(s)+\int_{\bar{\Lambda}^{+}(1)}^{\infty} \varphi_{+}(s) \mathrm{d}\left\{M_{+}(s)-s\right\} \\
\quad-\int_{0}^{\bar{\Lambda}^{-}(1)} \varphi_{-}(s) \mathrm{d} M_{-}(s)-\int_{\bar{\Lambda}^{-}(1)}^{\infty} \varphi_{-}(s) \mathrm{d}\left\{M_{-}(s)-s\right\}
\end{aligned}
$$

and

Then

$$
S:=\int_{0}^{\infty} \varphi_{+}^{2}(s) \mathrm{d} M_{+}(s)+\int_{0}^{\infty} \varphi_{-}^{2}(s) \mathrm{d} M_{-}(s)
$$

$$
\begin{aligned}
& E\left(e^{\mathrm{i}\left(\theta_{1} W+\theta_{2} S\right)}\right) \\
= & E\left(e^{\mathrm{i} V_{+}\left(\theta_{1}, \theta_{2}\right)} e^{\mathrm{i} V_{-}\left(\theta_{1}, \theta_{2}\right)}\right) \exp \left(\int_{\bar{\Lambda}^{+}(1)}^{\infty} \varphi_{+}^{2}(s) \mathrm{d} s+\int_{\bar{\Lambda}^{-}(1)}^{\infty} \varphi_{-}^{2}(s) \mathrm{d} s\right) \\
= & \phi_{+}\left(\theta_{1}, \theta_{2}\right) \phi_{-}\left(\theta_{1}, \theta_{2}\right) \exp \left(\int_{\{0<|x| \leq 1\}} x^{2} \Lambda(\mathrm{d} x)\right) \\
= & \phi\left(\theta_{1}, \theta_{2}\right) \exp \left(\int_{\{0<|x| \leq 1\}} x^{2} \Lambda(\mathrm{d} x)\right) .
\end{aligned}
$$

Putting the primes back now, we see that the right-hand side of (5.46) has bivariate characteristic function

$$
e^{\mathrm{i} \theta_{1} \beta^{\prime}-\frac{1}{2} \theta_{1}^{2}\left(\tau^{\prime}\right)^{2}+\mathrm{i} \theta_{2}\left(\tau^{\prime}\right)^{2}} E\left(e^{\mathrm{i}\left(\theta_{1} W+\theta_{2} S\right)}\right),
$$

so we conclude that

$$
\left(\beta^{\prime}+\tau^{\prime} Z+\mathcal{I}^{\prime},\left(\tau^{\prime}\right)^{2}+\mathcal{J}^{\prime}\right) \stackrel{\mathrm{D}}{=}\left(\beta^{\prime}+\tau^{\prime} Z+W,\left(\tau^{\prime}\right)^{2}+S\right)
$$

where $Z$ is a standard normal $\mathrm{rv}$ independent of $(W, S)$, and $\tau^{\prime} \geq 0$. (5.46) gives

$$
\frac{X_{t_{k^{\prime}}}}{\sqrt{V_{t_{k^{\prime}}}}} \stackrel{\mathrm{D}}{\longrightarrow} \frac{\beta^{\prime}+\tau^{\prime} Z+\mathcal{I}^{\prime}}{\sqrt{\left(\tau^{\prime}\right)^{2}+\mathcal{J}^{\prime}}}
$$

where the right-hand side is $N(0,1)$ by assumption. We have the setup of Theorem 1 of Mason [35, so we can deduce that we must have $\Lambda^{\prime} \equiv 0=\beta^{\prime}$, and $\tau^{\prime}>0$ in 
(5.50). This means that $X_{t_{k^{\prime}}} / b\left(t_{k^{\prime}}\right) \stackrel{\mathrm{D}}{\longrightarrow} N\left(0,\left(\tau^{\prime}\right)^{2}\right)$, where $\tau^{\prime}>0$, and this implies $X_{t} \in D_{0}(N)$ by Part (iv) of Theorem 2.4 completing this part of the proof.

Now assume that (2.16) holds. This implies $\lim _{x \rightarrow \infty} x^{2} \bar{\Pi}(x) / V(x)=0$, and so $V(x) \sim U(x)$ as $x \rightarrow \infty$, and moreover, $V(x)$ and thus $U(x)$ are slowly varying at zero. Thus with $b(t)$ satisfying (4.24) with $\lambda=1$, we get for any $0<\varepsilon \leq 1$,

$$
\frac{t V(\varepsilon b(t))}{b(t)} \rightarrow 1, \text { as } t \downarrow 0,
$$

which implies (4.3) with $\tau^{2}=1$ in Proposition 4.1, In addition, we have by (2.16) that

and for all $x>0$,

$$
\frac{t \nu(b(t))}{b(t)} \rightarrow 0, \text { as } t \downarrow 0,
$$

$$
t \bar{\Pi}(x b(t)) \rightarrow 0, \text { as } t \downarrow 0,
$$

where we also use the fact that $U$ is slowly varying at zero. Hence we have (4.4), in which $\Lambda \equiv 0$, so $\mathcal{I}_{1}=\mathcal{J}_{1}=0$ a.s., and $\tau=1$. Thus

$$
\left(\frac{X_{t}}{b(t)}, \frac{V_{t}^{2}}{b^{2}(t)}\right) \stackrel{\mathrm{D}}{\longrightarrow}(Z, 1), \text { as } t \downarrow 0,
$$

which implies $X_{t} / \sqrt{V_{t}} \stackrel{\mathrm{D}}{\longrightarrow} N(0,1)$, as $t \downarrow 0$.

Remark. Note that in the course of the proof of Theorem 3.6 we proved the following:

Proposition 5.7. Whenever a pair of random variables $(X, V)$ has joint characteristic function (5.47), it has the distributional representation

$$
(X, V) \stackrel{\mathrm{D}}{=}\left(\beta^{\prime}+\tau^{\prime} Z+W,\left(\tau^{\prime}\right)^{2}+S\right)
$$

where $Z$ is a standard normal rv independent of $(W, S)$ and $\tau^{\prime} \geq 0$.

\section{REFERENCES}

1. Barndorff-Nielsen, O.E, Mikosch, T., and Resnick, S.I. (2001) Lévy Processes: Theory and Applications, Birkhäuser, Boston. MR1833689 (2001m:60004)

2. Beichelt, F. (2006) Stochastic Processes in Science, Engineering and Finance, CRC Press, Boca Raton, FL. MR2207217

3. Bertoin, J. Lévy Processes. (1996) Cambridge University Press, Cambridge. MR.1406564 (98e:60117)

4. Bertoin, J., Doney, R.A., and Maller, R.A. (2008) Passage of Lévy Processes across Power Law Boundaries at Small Times, Ann. Probab., 36, 160-197. MR2370602 (2009d:60141)

5. Bingham, N.H., Goldie, C.M. and Teugels, J.L. (1987) Regular Variation. Encyclopedia of Mathematics and its Applications, 27, Cambridge University Press, Cambridge. MR 898871 (88i:26004)

6. Breymann, W., Dias, A., and Embrechts, P. (2003) Dependence structures for multivariate high-frequency data in finance. Quantitative Finance 3, 1-16. MR.1972372

7. Blumenthal, R.M. and Getoor, R.K. (1961) Sample functions of stochastic processes with stationary independent increments. J. Math. Mech., 10, 492-516. MR0123362 (23:A689)

8. Buchmann, B., Maller, R.A., and Szimayer, A. (2008) An almost sure functional limit theorem at zero for a Lévy process normed by the square root function, and applications, Prob. Theor. Rel. Fields, 142, 219-247. MR 2413271

9. Chistyakov, G.P. and Götze, F. (2004) Limit distributions of studentized sums. Ann. Probab., 32, 28-77. MR2040775 (2005f:60055)

10. de la Peña, V.H., Lai, T.L. and Shao, Q.-M. (2009) Self-Normalized Processes: Limit Theory and Statistical Applications. Springer-Verlag, Berlin 
11. Doney, R.A. and Maller, R.A. (2002) Stability and attraction to normality for Lévy processes at zero and infinity. J. Theoretical Probab., 15, 751-792. MR1922446 (2003g:60076)

12. Doney, R.A. and Maller, R.A. (2002) Stability of the overshoot for Lévy processes, Ann. Prob., 30, 188-212. MR.1894105 (2003d:60090)

13. Doney, R. A. and Maller, R.A. (2005) Passage times of random walks and Lévy processes across power law boundaries. Prob. Theor. Rel. Fields. 133, 57-70. MR2197137 (2007f:60038)

14. Erickson, K.B., and Maller, R.A. (2007) Finiteness of integrals of functions of Lévy processes, Proc. Lond. Math. Soc., 94, 386-420. MR2308232 (2008g:60140)

15. Feller, W. (1966) On regular variation and local limit theorems. In: Proc. Fifth Berkeley Symp. Math. Statist. Prob., 2, 373-388. Univ. California Press, Berkeley. MR0219117 (36:2200)

16. Feller, W. (1971) An Introduction to Probability Theory and its Applications, 2nd Ed., Wiley, NY. MR0270403(42:5292)

17. Gikhman, I.I. and Skorokhod, A.V. (1974) The Theory of Random Processes, Springer-Verlag, Berlin, NY. MR0651014 (58:31323a)

18. Giné, E., Götze, F., and Mason, D.M. (1997) When is the student $t$-statistic asymptotically standard normal? Ann. Prob. 25, 1514-1531. MR1457629 (98j:60033)

19. Giné, E. and Mason, D.M. (1998) On the LIL for Self-Normalized Sums of IID Random Variables, J. Theoretical Probab., 11, 351-370. MR.1622575 (99e:60082)

20. Goldie, C.M. and Maller, R.A. (1998) Generalised densities of order statistics. Statistica Neerlandica, 53, 222-246. MR 1708011 (2000f:62118)

21. Griffin, P.S. (2002) Tightness of the Student t-statistic, Electron. Comm. in Probab. 7, 181190. MR 1937903 (2003i:60037)

22. Griffin, P.S. and Maller, R.A. (1998) On the rate of growth of the overshoot and the maximal partial sum. Adv. Appl. Prob. 30, 1-16. MR.1618833 (99c:60149)

23. Griffin, P.S. and Maller, R.A. (1999a) On compactness properties of the exit position of a random walk from an interval, Proc. Lond. Math. Soc., 78, 459-480. MR1665250 (2000e:60147)

24. Griffin, P.S. and Maller, R.A. (1999b) Dominance of the sum over the maximum and some new classes of stochastic compactness. Invited Paper, In: Perplexing Problems in Probability, Festschrift in Honor of Harry Kesten, M. Bramson and R. Durrett, Eds., Progress in Probability 44 (Birkhäuser, Boston, 1999), 219-246. MR1703134 (2000k:60035)

25. Griffin, P.S., and Mason, D.M. (1991). On the asymptotic normality of self-normalized sums, Proc. Cambridge Phil. Soc. 109, 597-610. MR1094756 (92d:60029)

26. Gut, A. (2006) Gnedenko-Raikov's theorem, central limit theory, and the weak law of large numbers, Statistics \& Prob. Letters, 76, 1935-1939. MR.2271190 (2007k:60066)

27. Kallenberg, O. (2002) Foundations of Modern Probability, 2nd Ed., Springer. MR1876169 (2002m:60002)

28. Kesten, H. and Maller, R.A. (1992) Ratios of trimmed sums and order statistics, Ann. Probab., 20, 1805-1842. MR.1188043 (94a:60045)

29. Lindvall, T. (1973) Weak convergence of probability measures and random functions in the function space $D[0, \infty)$. J. Appl, Prob., 10, 109-121. MR0362429 (50:14870)

30. Logan, B.F., Mallows, C.L., Rice, S.O. and Shepp, L. (1973) Limit distributions of selfnormalized sums. Ann. Probab. 1, 788-809. MR0362449 (50:14890)

31. Madan, D.B. and Seneta, E. (1990) The Variance Gamma (V.G.) model for share market returns. J. Business, 63, 511-524.

32. Maller, R.A. (1981) Some properties of stochastic compactness. J. Austral. Math. Soc., 30, 264-277. MR614077 (82h:60043)

33. Maller, R.A. (1981) A theorem on products of random variables with application to regression. Austral. J. Statist., 23, 177-185. MR636133 (82m:60032)

34. Maller, R.A. and Mason, D.M. (2008) Convergence in distribution of Lévy processes at small times with self-normalisation, Acta Sci. Math. (Szeged), 74, 315-347. MR2431109

35. Mason, D.M. (2005) The asymptotic distribution of self-normalized triangular arrays. J. Theor. Probab., 18, 853-870 MR2289935 (2008m:60035)

36. Mason, D.M. and Zinn, J. (2005) When does a randomly weighted self-normalized sum converge in distribution? Electronic Comm. Prob., 10, 70-81. MR.2133894 (2005m:60045)

37. Pruitt, W.E. (1981) The growth of random walks and Lévy processes. Ann. Probab. 9, 948956. MR632968 (84h:60063)

38. Raikov, D.A., (1938) On a connection between the central limit theorem in the theory of probability and the law of large numbers. Izvestiya Akad. Nauk SSSR Ser. Mat., 323-338. 
39. Sato, K. (1999) Lévy Processes and Infinitely Divisible Distributions. Cambridge University Press, Cambridge. MR.1739520 (2003b:60064)

40. Woerner, J. (2007) Inference in Lévy-type stochastic volatility models, Adv. Appl. Prob. 39, 531-549. MR2343676

Centre for Mathematical Analysis \& School of Finance and Applied Statistics, Australian National University, PO Canberra, ACT, Australia

E-mail address: Ross.Maller@anu.edu.au

Food and Resource Economics, University of Delaware, 206 Townsend Hall, Newark, DELAWARE 19717

E-mail address: davidm@Udel.Edu 